Magnetization studies of oxides related to the high temperature cuprate superconductors

by

Wang, Zhaorong

PHD Thesis submitted to Iowa State University

Ames Laboratory, U.S. DOE

Iowa State University

Ames, Iowa 50011

Date Transmitted: June 19, 1995

PREPARED FOR THE U.S. DEPARTMENT OF ENERGY

UNDER CONTRACT NO. W-7405-Eng-82.

DISTRIBUTION OF THIS DOCUMENT IS UNLIMITED 


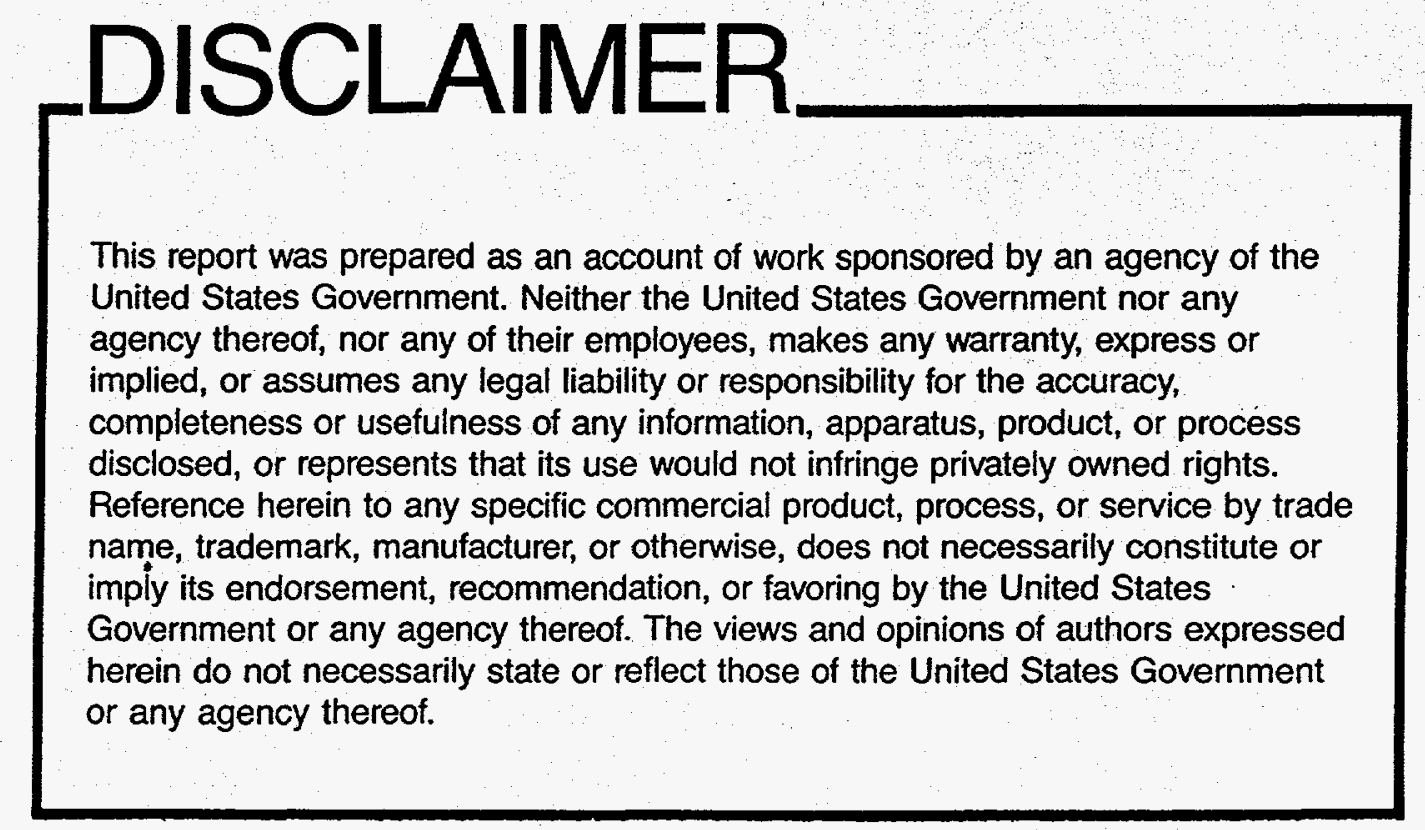




\section{DISCLAIMER}

Portions of this document may be illegible in electronic image products. Images are produced from the best available original document. 


\title{
Magnetization studies of
}

oxides related to the high temperature cuprate superconductors

by

\section{Zhaorong Wang}

\author{
A Dissertation Submitted to the \\ Graduate Faculty in Partial Fulfillment of the \\ Requirements for the Degree of \\ DOCTOR OF PHILOSOPHY \\ Department: Physics and Astronomy \\ Major: Condesed Matter Physics
}

Approved:

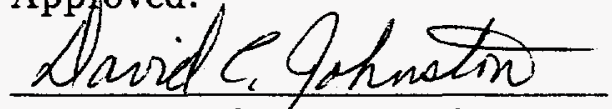

In Charge of Major Work

D/F Finimenus

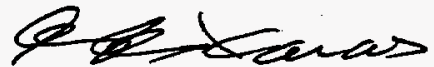

For the Graduate College

Iowa State University

Ames, Iowa

1994 
Magnetization studies of

oxides related to the high temperature cuprate superconductors

Zhaorong Wang

Major Professor: David C. Johnston
Iowa State University

An Oxford Instruments Faraday magnetometer, capable of operating in the temperature range between $1.5 \mathrm{~K}$ and $1000 \mathrm{~K}$, and magnetic field range between $0 \mathrm{~T}$ to 8 $\mathrm{T}$, with a sensitivity of $3 \times 10^{-8} \mathrm{~cm}^{3} \mathrm{G}$, was assembled and calibrated. The pumping station which supported this magnetometer was designed and built. Software for reducing the raw data and generating calibration files was written. The magnetic properties of several oxides related to the high temperature cuprate superconductors $\mathrm{BaCuO}_{2+x}, \mathrm{La}_{2} \mathrm{CuO}_{4}, \mathrm{Sr}_{2} \mathrm{IrO}_{4}, \mathrm{Sr}_{2} \mathrm{RhO}_{4}, \mathrm{Sr}_{2} \mathrm{VO}_{4}$, and $\mathrm{Sr}_{2} \mathrm{CuO}_{3}$ were measured using the Faraday magnetometer.

AC and DC magnetization, neutron diffraction, and heat capacity measurements on polycrystalline $\mathrm{BaCuO}_{2+x}$ revealed a combination of magnetic behaviors. The $\mathrm{Cu}_{6}$ ring clusters and $\mathrm{Cu}_{18}$ sphere clusters in this compound were found to have ferromagnetic ground states with large spins 3 and 9 , respectively. The $\mathrm{Cu}_{6}$ rings ordered antiferromagnetically below the Néel temperature $\mathrm{T}_{N}=15 \pm 0.5 \mathrm{Kelvin}$, whereas the $\mathrm{Cu}_{18}$ spheres remained paramagnetic down to 2 Kelvin. The ordered moment below $\mathrm{T}_{N}$ was $0.89(5)$ Bohr magnetons per $\mathrm{Cu}$ in the $\mathrm{Cu}_{6}$ rings, demonstrating that quantum fluctuation effects are small in these atomic clusters. The $\mathrm{Cu}_{18}$ clusters are predicted to exhibit ferromagnetic intercluster order below about 1 Kelvin. Heat capacity $C_{P}$ data for $\mathrm{BaCuO}_{2+x}$ show a sharp second order transition at the Néel 
temperature. The value of the discontinuity in the heat capacity at Néel is close to that expected for antiferromagentic ordering of the ring clusters, according to the mean field theory. An additional maximum in $C_{P}$ at about $0.8 \mathrm{~K}$ may correspond to the ferromagnetic ordering of the sphere clusters postulated above. Understanding the magnetic behavior of $\mathrm{BaCuO}_{2+x}$ is relevant to the pairing mechanism in high $\mathrm{T}_{c}$ cuprates.

Magnetic susceptibility measurements for $\mathrm{Sr}_{2} \mathrm{CuO}_{3} \pm \delta$ were made from $2 \mathrm{~K}$ to $800 \mathrm{~K}$, and a strong dependence upon oxygen content $(\delta)$ was observed. High temperature magnetic susceptibility measurements for the sample with the smallest Curie-Weiss-type term clearly show the increase with temperature expected from the Bonner-Fisher model for a spin-1/2 one dimensional (1-D) Heisenberg antiferromagnet. This is the first direct experimental observation of 1-D magnetic behavior in this system. Estimates of the interchain magnetic magnetic interaction and low temperature neutron and synchrotron $\mathrm{x}$-ray powder diffraction studies of $\mathrm{Sr}_{2} \mathrm{CuO}_{3}$ indicate that this material may be the best (nearly ideal) realization of a 1-D spin $1 / 2$ Heisenberg antiferromagnet reported to date. 


\section{Magnetization studies of}

oxides related to the high temperature cuprate superconductors

$$
\text { by }
$$

\section{Zhaorong Wang}

A Dissertation Submitted to the

Graduate Faculty in Partial Fulfillment of the

Requirements for the Degree of

DOCTOR OF PHILOSOPHY

Department: Physics and Astronomy

Major: Condesed Matter Physics

Approved:

In Charge of Major Work

For the Major Department

For the Graduate College

Iowa State University

Ames, Iowa

1994 


\section{TABLE OF CONTENTS}

ACKNOWLEDGMENTS $\ldots \ldots \ldots \ldots \ldots \ldots \ldots \ldots \ldots$ xv

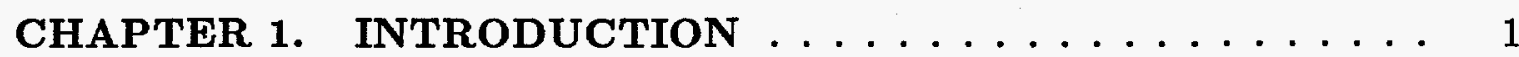

Basic Properties of Superconducting Materials . . . . . . . . . 6

Ginzburg-Landau Theory for Superconductivity . . . . . . . . . . 9

Landau Theory $\ldots \ldots \ldots \ldots \ldots \ldots \ldots \ldots$

Ginzburg-Landau Theory for Superconductivity . . . . . . . . 10

Coherence Length . . . . . . . . . . . . . . . 11

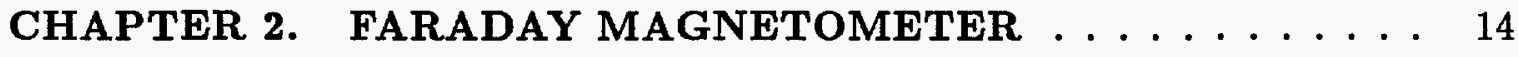

Introduction . . . . . . . . . . . . . . . . 14

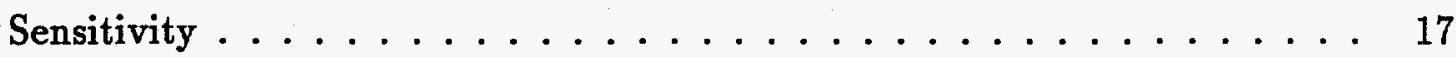

Pumping Station $\ldots \ldots \ldots \ldots \ldots \ldots \ldots \ldots \ldots \ldots \ldots \ldots \ldots \ldots \ldots$

Faraday Magnetometer Operation . . . . . . . . . . . . 21

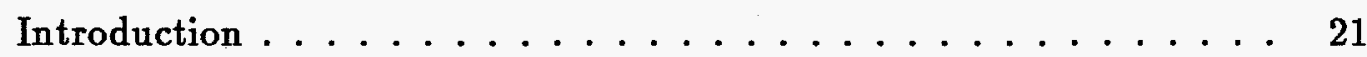

Magnetic Moment Measurement Sequence . . . . . . . . . . 24

Units . . . . . . . . . . . . . . . . 25

Sample Holder Contribution and Demagnetization Factor . . . . . 27

Calibration of Gradient Magnetic Field . . . . . . . . . . . 28

Centering the Sample in the Magnetometer . . . . . . . . . . 34 
Temperature Calibration . . . . . . . . . . . 35

\section{CHAPTER 3. SUPERCONDUCTING PROPERTIES OF}

$\mathrm{Ba}_{0.625} \mathrm{~K}_{0.375} \mathrm{BiO}_{3} \ldots \ldots \ldots \ldots \ldots \ldots \ldots \ldots \ldots \ldots \ldots \ldots$

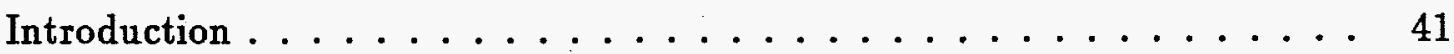

Sample Preparation and Charactization . . . . . . . . . . 42

Experimental Results . . . . . . . . . . . . . . . . 43

${ }^{+}{ }^{+}$SR Measurement $\ldots \ldots \ldots \ldots \ldots \ldots \ldots$

Magnetization Measurement . . . . . . . . . . . 44

Theory and Data Analysis . . . . . . . . . . . . . . 48

${ }{ }^{+} \mathrm{SR} \ldots \ldots \ldots \ldots \ldots \ldots \ldots \ldots \ldots \ldots \ldots \ldots \ldots \ldots$

Reversible Magnetization and Penetration Depth $\ldots \ldots \ldots \ldots 48$

Discussion . . . . . . . . . . . . . . . . 49

CHAPTER 4. MAGNETIC PROPERTIES OF $\mathrm{BaCuO}_{2+x} \ldots \ldots \quad 54$

Introduction . . . . . . . . . . . . . . 54

Experimental Details . . . . . . . . . . . . . . . . 59

Sample Preparation and Oxygen Content . . . . . . . . 59

X-ray Analysis $\ldots \ldots \ldots \ldots \ldots \ldots \ldots \ldots \ldots$

DC and AC Magnetization Measurements $\ldots \ldots \ldots \ldots 62$

Experimental Results and Preliminary Analysis . . . . . . . . 62

He-annealed $\mathrm{BaCuO}_{2+x} \ldots \ldots \ldots \ldots \ldots \ldots \ldots$

Oxygen-annealed $\mathrm{BaCuO}_{2+x} \ldots \ldots \ldots \ldots \ldots$

Detailed Analysis and Modeling for Helium-annealed Sample . . . . . . 70

Magnetization versus Magnetic Field and Temperature for $T \ll T_{N} \cdot \quad 70$

Low Temperature $\left(T \ll T_{N} \approx 15 \mathrm{~K}\right)$ Magnetic Susceptibility $\ldots .80$ 
High Temperature ( $\geq 40 \mathrm{~K}$ ) Susceptibility and Intracluster Coupling Constants ................... 81

Summary and Conclusions . . . . . . . . . . . . . 84

Neutron Diffraction Study of Magnetic Ordering of Helium-annealed Sample 86

Heat Capacity Study of Magnetic Ordering of Helium-annealed Sample . . 91

CHAPTER 5. MAGNETIC SUSCEPTIBILITY OF $\mathrm{Sr}_{2} \mathrm{CuO}_{3} \ldots 94$

Introduction . . . . . . . . . . . . . . . . . 94

Experimental Details . . . . . . . . . . . . . . . . 95

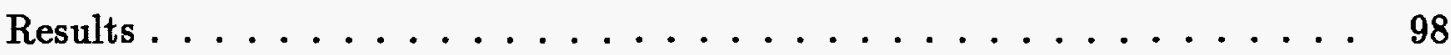

Synchrotron and Neutron Diffraction Studies . . . . . . . 98

Magnetic Susceptibility . . . . . . . . . . . . . 100

Data Analysis . . . . . . . . . . . . . . . 106

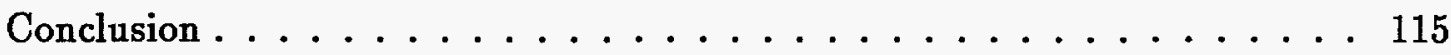

CHAPTER 6. MAGNETIC PROPERTIES OF $\mathrm{Sr}_{2} \mathrm{IrO}_{4} \ldots \ldots 117$

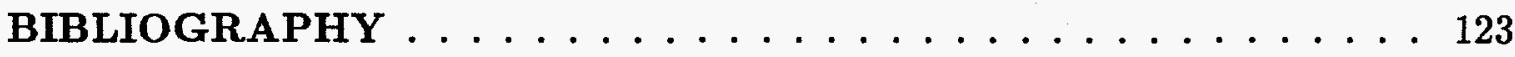




\section{LIST OF FIGURES}

Figure 2.1: Sketch of the Oxford Instruments Faraday magnetometer . .

Figure 2.2: The pumping diagram for the Faraday magnetometer. The pump system in room A-206 consists of two separate pumping systems. The pump in the low temperature lab is connected to the variable temperature insert through valve 2 . The high vacuum pumping station in room A 206 is connected to the sample space through valves 1 and 3 . The pressure in the sample space is monitored by gauges 1,2 , and 3 . The pressure in the variable temperature insert space is monitored by gauges 4 and $5 . \ldots \ldots \ldots \ldots \ldots$

Figure 2.3: The calibration factor calif versus magnetic field at different gradient coil currents for the calibration data file zrwcalif.cal, where the symbols represent the value of gradient coil current

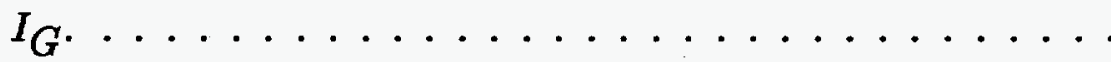


Figure 2.4: (a) The susceptibility determined according to constant calibration factor calif $=0.0341$ versus magnetic field at different gradient fields for a standard Ta sample and its sample holder. (b) The susceptibility, determined according to calibration file zrwcalif.cal, versus magnetic field at different gradient fields for a standard Ta sample and its sample holder. The symbols represent various values of the gradient coil current $I_{G}$. . .

Figure 2.5: (a) The susceptibility determined according to a bipolynomial (Oxford Instruments' program) versus magnetic field at different gradient fields for a standard Ta sample and its sample holder. (b) The susceptibility, determined according to calibration file zrwcalif.cal, versus magnetic field at different gradient fields for a standard Ta sample corrected for the sample holder signal. The symbols represent the values of the gradient coil current $I_{G} \ldots \ldots \ldots \ldots \ldots \ldots$

Figure 2.6: The magnetization versus temperature for four different standard calibration samples for the calibration of temperature .

Figure 2.7: The magnetization versus temperature for a standard calibration sample of Alumel.

Figure 2.8: The magnetization versus temperature for a standard calibration sample of nickel. . . . . . . . . . . . . 38

Figure 2.9: The magnetization versus temperature for a standard calibration sample of Nicoseal. . . . . . . . . . . . . . 
Figure 2.10: The magnetization versus temperature for a standard calibration sample of Perkalloy. . . . . . . . . . . . . 40

Figure 3.1: Temperature dependence of the Gaussian relaxation rate $\sigma$ and Larmor frequency for an applied field $H_{e x t}=4 \mathrm{kG}$. .

Figure 3.2: $\quad$ Magentization $M$ versus applied magnetic field $H$ at different temperatures $T$. The solid and dashed lines are the theoretical fits to the experimental points. . . . . . . . . . 46

Figure 3.3: $\quad$ Magnetization $M$ versus Temperature $T$ at different magnetic

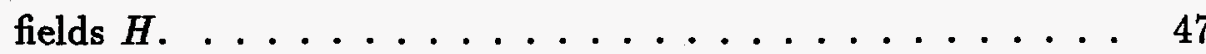

Figure 3.4: Penetration depth $\lambda$ versus temperature. The solid dots are $\mu \mathrm{SR}$ data, and the open circles are magnetization data. . . . 50

Figure 3.5: $\quad$ Upper critical field $H_{c 2}$ versus temperature $\ldots \ldots$. . . . 51

Figure 4.1: Perspective representation of the two types of $\mathrm{Cu}-\mathrm{O}$ clusters in the bcc unit cell of $\mathrm{BaCuO}_{2}+x$. The sphere-like clusters are located at the $(000)$ and $(1 / 21 / 21 / 2)$ (not shown) the ringlike clusters are located at the $(1 / 41 / 41 / 4)$ and the remaining seven equivalent positions with their axis of highest symmetry along the corresponding body diagonal (only two rings are shown). The lone spins are located along principal directions adjacent to the spheres (partially occupied). Both clusters consist of closed one-dimensional strips of $\mathrm{Cu}_{4} \mathrm{O}$ oxygen edgesharing squares. .................. 57

Figure 4.2: Thermogravimeteric analysis (TGA) data for $\mathrm{BaCuO}_{2+x} \ldots 61$ 
viii

Figure 4.3: Powder x-ray diffraction patterns for $\mathrm{BaCuO}_{2+x}$. . . . 63

Figure 4.4: Inverse magnetic susceptibility $\chi^{-1}$ versus temperature for $\mathrm{He}$-annealed $\mathrm{BaCuO}_{2+x}$ between $2 \mathrm{~K}$ to $400 \mathrm{~K}$. . . . . 65

Figure 4.5: Top. Magnetization vs. applied magnetic field $H$ at various temperatures for $\mathrm{He}$-annealed $\mathrm{BaCuO}_{2+x}$. Bottom. Slope $\chi_{o}$ (right-hand-scale) and y-intercept $M_{0}$ (left-hand scale) of straight-line fits to the data in the top panel between $30 \mathrm{kG}$ and $55 \mathrm{kG} \ldots \ldots \ldots \ldots \ldots \ldots$

Figure 4.6: High field ( $H=55 \mathrm{kG}$ ) DC magnetization $M$ divided by $H$ versus temperature for $\mathrm{He}$-annealed $\mathrm{BaCuO}_{2+x}$ (open circles). The temperature derivative of these data versus temperature (open squares) is also shown. . . . . . . . .

Figure 4.7: AC magnetic susceptibility $\chi^{\prime}$ in various DC fields versus temperature. The open triangles are the high-field differential susceptibility data $\chi_{o}(T)$ reproduced from Fig. 4.5(Bottom).

Figure 4.8: Inverse magnetic susceptibility $\chi^{-1}$ versus temperature for $\mathrm{O}_{2}$-annealed $\mathrm{BaCuO}_{2+x}$ between $2 \mathrm{~K}$ and $400 \mathrm{~K}$. The solid line is a fit of Eq. (4.1) to the data above $300 \mathrm{~K}$. . . . . .

Figure 4.9: $\quad$ Top. Magnetization versus applied magnetic field $H$ at various temperatures for $\mathrm{O}_{2}$-annealed $\mathrm{BaCuO}_{2+x}$. Bottom. Slope $\chi_{o}$ (right-hand-scale) and y-intercept $M_{o}$ (left-hand-scale) of straight-line fits to the data in top panel between $30 \mathrm{kG}$ and

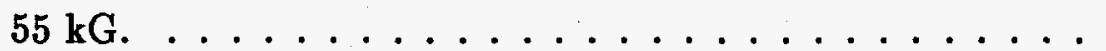


Figure 4.10: The saturating part $M(H)-\chi_{o} H$ of the magnetization $M(H)$ at $2 \mathrm{~K}$ for $\mathrm{He}$-annealed $\mathrm{BaCuO}_{2+x}$, from Fig. 4.5(Top), normalized to $M(H=37 \mathrm{kG})-\chi_{o}(37 \mathrm{kG})$, open circles, plotted versus reduced magnetic field $\mu_{B} H / k_{B}(T-\theta)$, where $\theta=0.3$ K. Brillouin functions with $T$ replaced by $T-\theta, g=2$ and spins $S=6,9$ and 15 are also plotted versus reduced field. . .

Figure 4.11: Magnetization $(M)$ versus applied magnetic field $(H)$ for Heannealed $\mathrm{BaCuO}_{2+x}$ (open circles). The solid curve labeled Sum is a theoretical fit to the data [Eq. (4.12)]. The contributions to the Sum from the lone $\mathrm{Cu}$ ions, the $\mathrm{Cu}_{6}$ clusters, and the $\mathrm{Cu}_{18}$ sphere clusters are as indicated. . . . . . .

Figure 4.12: Inverse magnetic susceptibility $\chi^{-1}$ versus temperature $T$ for $\mathrm{He}$-annealed $\mathrm{BaCuO}_{2+x}$ (open squares), reproduced from Fig. 4.4(Bottom). The solid curve is the predication of $\chi^{-1}$ for the lone $\mathrm{Cu}$ ions and $\mathrm{Cu}_{18}$ sphere clusters. The filled diamonds are the derived $\chi_{r}(T)$ data for the $\mathrm{Cu}_{6}$ rings. $\quad \ldots \quad 82$ 
Figure 4.13: Top. Inverse magnetic susceptibility $\chi^{-1}$ versus temperature for He-annealed $\mathrm{BaCuO}_{2+x}$, reproduced from Fig. 4.4(Top). The solid curve is a theoretical fit [Eq. (4.16)] to the data above $70 \mathrm{~K}$. Bottom. Theoretical predictions for the inverse susceptibility $\chi^{-1}$ of the $\mathrm{Cu}_{6}$ rings versus temperature. The solid curve is the prediction using Eq. (4.21) and the approximate cluster magnetic energy levels in Eq. (4.20). The results of exact calculations using the Heisenberg model are shown by the various symbols for the corresponding values of exchange constant $J_{r} \ldots \ldots \ldots \ldots \ldots \ldots \ldots \ldots \ldots$

Figure 4.14: Unpolarized-neutron scans for the $(1,1,1)$ reflection at temperatures of $4.2 \mathrm{~K}$ and $90 \mathrm{~K}$. The solid curve for each scan is a multiple Gaussian fit to the experimental data. Inset: temperature dependence of the integrated intensity of the $(1,1$, 1) reflection (the solid curve is a guide to the eye). . . . . .

Figure 4.15: Polarized-neutron scans at $T=4.2 \mathrm{~K}$. The top and the bottom scans show the spin-flip (magnetic) and the non-spin-flip (nuclear) scattering, respectively. The solid curves are guides

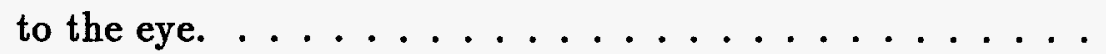

Figure 4.16: Heat capacity divided by temperature $C / T$, versus temperature. In the top panel, data were obtained in magnetic fields $H=0$ (open circles) and $70 \mathrm{kG}$ (filled circles). . . . . .

Figure 4.17: Heat capacity $C$ versus temperature for applied field $H=0$ (filled circles) and $H=7 \mathrm{~T}$ (open circles). . . . . . . 
Figure 5.1: The crystal structure of $\mathrm{Sr}_{2} \mathrm{CuO}_{3}$. The $\mathrm{Cu}-\mathrm{O}$ chains are parallel to the a axis. . . . . . . . . . . 96

Figure 5.2: $\quad$ Magnetic susceptibility $\chi$ versus temperature for the first time running (upper curve) and second time running (lower curve). 97

Figure 5.3: Magnetization versus magnetic field isotherms at different temperatures T. . . . . . . . . . . .

Figure 5.4: Powder neutron diffraction data collected at $11 \mathrm{~K}$. The results of Rietveld refinement are superimposed on the raw data. The vertical tick marks indicate the expected location of diffraction peaks. The fit residuals are plotted at the bottom of the figure. The neutron wavelength was $1.5401 \AA . \ldots \ldots 101$

Figure 5.5: Synchrotron $\mathrm{x}$-ray diffraction data collected at $12 \mathrm{~K}$. The vertical tick marks indicate the expected location of diffraction peaks. The $\mathrm{x}$-ray wavelength was $0.70377 \AA$. . . . . . . 102

Figure 5.6: The temperature $(T)$ dependencies of the magnetic susceptibilities $(\chi)$ for the samples annealed under different conditions. The diamagnetic core contributions $\left(\chi_{d i a}\right)$ have been subtracted. The measurement field was $1.0 \mathrm{~T}$ in each case. . 105

Figure 5.7: The magnetic susceptibility $\left(\chi-\chi_{d i a}\right)$ versus temperature up to $800 \mathrm{~K}$ for the sample annealed under 6 torr helium. The experimental data are represented by open circles, while the theoretical curves described in the text are shown by dotted lines. . . . . . . . . . . . . . . . . . . . 110 
Figure 6.1: (Top) Magnetic susceptibility versus temperature T, data measured with a Faraday balance from 5 to $650 \mathrm{~K}$. (Bottom) Magnetic hysteresis curve measured with a SQUID magnetometer at $5 \mathrm{~K}$. The arrows indicate the direction in which the field is changing. . . . . . . . . . . . . . 120 
xiii

\section{LIST OF TABLES}

Table 2.1: A Faraday magnetometer raw data file for a Pt sample of mass $196.57 \mathrm{mg}$, where $\mathrm{g}=980.5 \mathrm{~cm} / \mathrm{s}^{2} \ldots \ldots . . . . . .26$

Table 2.2: The Faraday magnetometer data file (for $\mathrm{H}=2000 \mathrm{G} \mathrm{G}=3$ A) for Pt with different units .............. 26

Table 4.1: Observed and calculated integrated intensities of the the mag- netic reflections at $\mathrm{T}=4.2 \mathrm{~K}$. The intensities of the magnetic reflections were normalized to a nuclear diffraction pattern of the angular $2 \theta$ range $8-60$ degrees, excluding the magnetic reflections. The magnetic moment determined from the model described in the text is $\mu=0.89 \pm 0.05 \mu_{B} \ldots \ldots \ldots$

Table 5.1: Low temperature crystallographic data for $\mathrm{Sr}_{2} \mathrm{CuO}_{3}$ determined by Rietveld refinement of neutron diffraction data obtained at $11 \mathrm{~K} \ldots \ldots \ldots \ldots$

Table 5.2: $\quad$ Parameters for $\mathrm{Sr}_{2} \mathrm{CuO}_{3+\delta}$ derived from iodometric titrations and magnetic refinement assuming that $g=2.1,|J| / \mathrm{k}_{\mathrm{B}}=$ 1307 K. . . . . . . . . . . . . . 104 
Table 5.3: $\quad \mathrm{J}, \mathrm{J}_{1}, \mathrm{~J}_{2}$ and $\mathrm{T}_{N}$ values for several linear chain antiferromagnets compared with the values for $\mathrm{Sr}_{2} \mathrm{CuO}_{3}$. Note that $\mathrm{J}_{1}$ and $\mathrm{J}_{2}$ for $\mathrm{Sr}_{2} \mathrm{CuO}_{3}$ are the values estimated assuming only dipolar coupling between the $\mathrm{Cu}^{2+}$ spins. . . . . . . . 112 


\section{ACKNOWLEDGMENTS}

I would like to express my deepest appreciation to my advisor Professor David C. Johnston for his guidance, patience, and understanding. His attitude of pursuing perfection as an experimentalist has had a revolutionary influence on both my professional and daily life.

I would also like to thank Dr. David Vaknin for introducing me to the $\mathrm{BaCuO}_{2+x}$ problem, thank Drs. X.-L Wang and J. A. Fernandez-Baca for their support on neutron experiments, and thank all of them for many stimulating discussions.

I would also like to thank Drs. M. K. Crawford, T. Ami and their associates for their $\mathrm{Sr}_{2} \mathrm{CuO}_{3}$ and $\mathrm{Sr}_{2} \mathrm{IrO}_{4}$ samples and for stimulating discussion in analyzing the magnetic data.

I would also like to thank Dr. Norman E. Phillips, Dr. Robert A. Fisher and their associates for specific heat measurments of my $\mathrm{BaCuO}_{2+x}$ sample, and their stimulating discussion.

I would also like to thank Dr. E. J. Ansaldo and Dr. T. Riseman for their measurement of my $\mathrm{Ba}_{0.625} \mathrm{~K}_{0.375} \mathrm{BiO}_{3}$ sample, and thank Drs. J. H. Cho, Z. D. Hao, J.R. Clem, V. Kogan, S. Mitra, and O. B. Hyun for stimulating discussion in analysis of magnetic data of that sample.

I would also like to thank Drs. H. Takeya, J. Moorman and K. A. Gschneidner, 
xvi

Jr for making it possible for me to make AC susceptibility measurements in their group.

I would also like to thank A. V. Mahajan, J. H. Oho, F. Chou, L. L. Miller, and W. Lee for teaching me many basic experimental techniques, and thank B. K. Che, S. Kondo, and S. Pace for numerous helpful discussions.

I would also like to thank K. M. Ho and C. T. Chan to let me use their workstation and softwares.

I would also like to thank Ames Lab. service department and Physics machine shop in helping me build the pumping station for the Faraday Magnetometer.

Ames Laboratory is operated for the U.S. Department of Energy by Iowa State University under Contract No. W-7405-Eng-82. This work was supported by the Director for Energy Research, Office of Basic Energy Sciences.

This thesis is dedicated to my father who passed away last year, my wife and our first baby who was born this year.

This work was performed at Ames Laboratory under Contract No. W-7405-eng-82 With the U.S. Department of Energy. The United States govemunt has assigned the DOE Report number IS -T1723 to this thesis. 


\section{CHAPTER 1. INTRODUCTION}

Due to the discovery of high temperature superconductivity [1] in 1986 in the $\mathrm{La}-\mathrm{Ba}-\mathrm{Cu}-\mathrm{O}$ system and many related materials, for example the $\mathrm{Y}-\mathrm{Ba}-\mathrm{Cu}-\mathrm{O}$ system [2], the traditional interpretation for the mechanism of superconductivity (electronphonon interaction) is no longer clearly applicable. So it is a time to review basic properties and the existing theory of superconductors, a time to test these new superconductors with new ideas and new technologies, a time to try to find more new types of superconductors and find new properties and interpretation associated with these new materials.

This dissertation is designed to make an attempt to study some of these points, and is composed of three basic components: First the new technology, which is described in Chapter 2 (the Faraday Magnetometer chapter); second the new ideas, described in the Chapter 1 (introduction chapter); third the new materials, described in Chapter 4 (the $\mathrm{BaCuO}_{2+x}$ chapter), 5 (the $\mathrm{Sr}_{2} \mathrm{CuO}_{3}$ chapter), and 6 (the $\mathrm{Sr}_{2} \mathrm{IrO}_{4}$ chapter).

This chapter includes a basic introduction to the thesis and a review of the basic properties of superconductors and the Ginzburg-Landau theory for superconductivity.

The superconductive state is characterized by several parameters, for example the penetration depth, correlation length, critical temperature, and critical field. 
High temperature superconductors have distinctive parameters, such as high critical field, small coherence length, large penetration depth, and of course high critical temperature. Furthermore the temperature dependence of the penetration depth will give us information on whether a material is in the BCS clean limit, dirty limit, or follows the two-fluid model, or must be described by a non-BCS theory: the low temperature behaviour of the penetration depth versus temperature gives us information about whether the material is in an s-wave or $d$-wave state, which reflects the symmetry of the superconducting order parameter.

The magnetic penetration depth $\lambda$ of $\mathrm{Ba}_{0.625} \mathrm{~K}_{0.375} \mathrm{BiO}_{3}$ was measured using both $\mathrm{DC}$ magnetization and $\mu \mathrm{SR}$ measurements. The temperature dependence of the magnetic penetration depth for both methods indicates conventional s-wave pairing with the isotropic extrapolated value $\lambda(0)=3400 \AA$, similar to that of $\mathrm{La}_{2-x} \mathrm{Sr}_{x} \mathrm{CuO}_{4}$ samples of the same superconducting transition temperature. However, as the temperature approaches the transition temperature, there is a significant difference between the magnetic penetration depth from these two methods, and a possible origin for the difference was postulated to be the magnetic field dependence of the penetration depth.

On the other hand, high temperature superconductors have their unique normal state behavior, spin one-half two-dimensional antiferromagnetism, and the structures of all known high transition temperature $\mathrm{T}_{c}$ cuprate superconductors contain $\mathrm{CuO}_{2}$ planes. The strong antiferromagnetic (AF) coupling between the $\mathrm{Cu}$ spins in the $\mathrm{CuO}_{2}$ planes of the undoped parent compounds of the high transition temperature cuprate superconductors results from an indirect $180^{\circ}$ bond angle $\mathrm{Cu}^{+2}-\mathrm{O}^{-2}-\mathrm{Cu}^{+2}$ superexchange interaction $(\mathrm{J} \approx 500 \mathrm{~K})$. It was argued that upon doping, an inter- 
vening $\mathrm{O}^{-1}$ ion produced by a localized doped hole on the $\mathrm{O}^{-2}$ ion results instead in an indirect ferromagnetic (FM) interaction between the two adjacent $\mathrm{Cu}$ spins. The ferromagnetic interaction would result in magnetic frustration which may be relevant to the superconducting pairing mechanism in the high $\mathrm{T}_{c}$ cuprates. An alternative cause of the FM interaction has been predicted to be a change in the $\mathrm{Cu}^{+2}-\mathrm{O}^{-2}-\mathrm{Cu}^{+2}$ bond angle from $180^{\circ}$ to $90^{\circ}$; however, the intermediate angle at which the crossover from AF to FM coupling occurs is unknown. Thus it is important to futher clarify the conditions under which $\mathrm{FM}$ versus $\mathrm{AF} \mathrm{Cu}^{+2}{ }_{-} \mathrm{Cu}^{+2}$ interactions occur in copper oxides.

Herein, we describe a detailed study of the magnetic properties of the compound $\mathrm{BaCuO}_{2+x}$. The compound has a large body-centered-cubic unit cell ( $a=18.25 \AA$ ) with a 90 formula units per unit cell. The cell contains 6 lone $\mathrm{CuO}_{4}$ units, $8 \mathrm{Cu}_{6} \mathrm{O}_{12}$ ring clusters and $2 \mathrm{Cu}_{18} \mathrm{O}_{24}$ sphere clusters formed from edge-shared $\mathrm{CuO}_{4}$ units. From unpolarized and polarized neutron diffraction measurements combined with magnetization measurements, we find that $\mathrm{BaCuO}_{2+x}$ exhibits a remarkable combination of novel magnetic behaviors. The $\mathrm{Cu}_{6}$ and $\mathrm{Cu}_{18}$ clusters have ferromagnetic ground states with large spins $S_{r}=3$ and $S_{s}=9$, respectively. The $\mathrm{Cu}_{6}$ rings exhibit long range antiferromagnetic intercluster order below $T_{N}=15 \mathrm{~K}$, with no apparent magnetic coupling to the lone $\mathrm{Cu}$ ions or the $\mathrm{Cu}_{18}$ clusters. In contrast, these latter two species remain paramagnetic down to $2 \mathrm{~K}$ and interact antiferromagnetically. Extrapolation of the magnetic susceptibility $\chi(T)$ data below $2 \mathrm{~K}$ predicts that the $\mathrm{Cu}_{18}$ clusters should exhibit ferromagnetic intercluster order below $\sim 1 \mathrm{~K}$. Our results are relevant to many cuprate superconductors which show buckling of the $\mathrm{CuO}_{2}$ planes and significant deviations of the $\mathrm{Cu}-\mathrm{O}-\mathrm{Cu}$ bond angle from $180^{\circ}$. 
By using a generalized ferrimagnetic model, the interaction strength between a sphere cluster ball and lone ion is found to be $1.2 \mathrm{meV}$. By applying the Van Vleck approximation to the Heisenberg model, a fit to the high temperature susceptibility data yielded the intrasphere coupling constant $J_{s}=38 \mathrm{~K}$ and intraring coupling constant $J_{r}=148 \mathrm{~K}$. The exact magnetic susceptibility of the $\mathrm{Cu}_{6}$ ring cluster was calculated by solving the Heisenberg model numerically, and the error bars for $J_{s}$ and $J_{r}$ gotten from the previous model were estimated.

The dimensionality appears to play an important role in high temperature superconducivity: all the high temperature cuprate superconductors contain two dimensional (2-D) $\mathrm{CuO}_{2}$ planes. It is natural to ask if 1-D cuprates exhibit superconductivity. If they do, what is the $\mathrm{T}_{c}$ of these systems, and if they donot, why not? $\mathrm{Sr}_{2} \mathrm{CuO}_{3}$, which has $\mathrm{CuO}$ chains with no $\mathrm{CuO}_{2}$ planes, becomes a superconductor after doping with a small amount of oxygen under pressure. Therefore it will be interesting to study in detail the magnetic behavior of this pure $\mathrm{CuO}$ chain material $\mathrm{Sr}_{2} \mathrm{CuO}_{3}$. Hopefully it will help us to understand the role of dimensionality in the magnetic properties of cuprates and its relation to the high $\mathrm{T}_{c}$ superconducting mechanism.

Therefore magnetic susceptibility measurements for $\mathrm{Sr}_{2} \mathrm{CuO}_{3} \pm \delta$ were made from $2 \mathrm{~K}$ to $800 \mathrm{~K}$, and a strong dependence upon oxygen content $(\delta)$ was observed. Samples synthesized under oxygen, followed by various nitrogen treatments, exhibited markedly different Curie-Weiss-type terms, and we discuss possible origins for this behavior. High temperature magnetic susceptibility measurements for the sample with the smallest Curie-Weiss-type term clearly showed the increase with temperature expected from the Bonner-Fisher model for a spin-1/2 one-dimensional Heisenberg 
antiferromagnet. This is the first direct experimental observation of 1-D magnetic behavior in this system. The in-chain superexchange coupling constant, as determined by a fit to the Bonner-Fisher model, is $|J| / \mathbf{k}_{B} \approx 1300 \pm 200 \mathrm{~K}$, comparable to the values observed in the two dimensional layered cuprates. Estimates of the interchain magnetic interaction strength indicate that this material may be the best realization of a 1-D spin 1/2 Heisenberg antiferromagnet reported to date. Low temperature neutron and synchrotron $\mathrm{x}$-ray powder diffraction studies of $\mathrm{Sr}_{2} \mathrm{CuO}_{3}$ indicate that the low temperature structure of this system has Immm space group symmetry, the same structure reported at room temperature, indicating that this material, in contrast to $\mathrm{La}_{2} \mathrm{CuO}_{4}$, does not ungergo any structural transformations upon cooling. The absence of crystallographic distortions precludes a magnetic anisotropy contribution from a Dzyaloshinsky-Moriya interaction, implying that $\mathrm{Sr}_{2} \mathrm{CuO}_{3}$ should be a nearly ideal spin $1 / 2$ antiferromagnetic Heisenberg chain compound, in agreement with the magnetic susceptibility results. A search for the presence of long range three dimensional antiferromagnetic order by magnetic neutron powder diffraction at temperatures as low as $1.5 \mathrm{~K}$ was not successful, although we estimate an upper limit for the size of the ordered moment which could have been detected to be $\sim 0.1 \mu_{B}$ per $\mathrm{Cu}^{+2}$ ion.

The discovery of high-temperature superconductivity in doped copper oxides, which in their insulating forms are nearly ideal two-dimensional spin-1/2 Heisenberg antiferromagnets, has stimulated great interest in finding additional examples of such magnetic systems. We find that $\mathrm{Sr}_{2} \mathrm{IrO}_{4}$, which has the $\mathrm{K}_{2} \mathrm{NiF}_{4}$ structure and should have a $5 \mathrm{~d}^{5}$ low-spin $(\mathrm{S}=1 / 2)$ electronic configuration, develops a ferromagnetic moment near $250 \mathrm{~K}$. The small size of the remanent moment $\left(10^{-2} \mu_{B}\right)$, and our 
structural studies, however, imply that this is weak ferromagnetism which appears at the Néel temperature due to a Dzyaloshinsky-Moriya interaction, in a manner analogous to that seen in $\mathrm{La}_{2} \mathrm{CuO}_{4}$.

\section{Basic Properties of Superconducting Materials}

There are usually five basic properties associated with superconducting materials: zero resistivity, critical field, Meissner effect, isotope effect, and the Josephson effect, as follows.

1) The resistivity of a superconducting material drops to zero when the temperature goes below a certain point called the transition temperature $\mathrm{T}_{c}$.

2) If an ellipsoidal shaped superconductor is placed into a magnetic field, the field not only will be excluded from superconductor, but also that field is expelled from an originally normal sample as it is cooled through the critical temperature. This latter phenomenon is called the Meissner effect.

3) If we increase the magnetic field in the superconducting state, after a critical point, the sample resistivity will become nonzero. That critical point is called the critical magnetic field, satisfying $H_{c}=\left[1-\left(\frac{T}{T_{c}}\right)^{2}\right]$. When the current density is above a critical value $J_{c}$ the sample will also become normal because of the field generated by the current, where $J_{c}=J_{0}\left[1-\left(\frac{T}{T_{c}}\right)^{2}\right]$. These are the behaviors of a so-called type I superconductor and the critical magnetic field is also called the thermodynamic critical field. However there are three different critical fields of type II superconductors: $H_{c 1}, H_{c 2}$, and $H_{c 3}$. In the range $H<H_{c 1}$, the field is excluded from the superconductor except for close to the surface; when $H_{c 1}<H<H_{c 2}$, the field penetrates the superconductor in the form of quantized flux; when $H_{c 2}$ 
$<H<H_{c 3}$, the field completely occupies the superconductor except for near the surface. The thermodynamic critical field in a type II superconductor $H_{c}$ is equal to $H_{c 2} /(\sqrt{2} \kappa)$, where $\kappa=\frac{\lambda}{\xi}, \lambda$ is the penetration depth and $\xi$ is the coherence length as defined below.

4) Another important property of superconductors is the isotope effect, which says that the critical temperature $T_{c}$ and the atomic mass $M$ has the following relation: $T_{c} \propto M^{-\frac{1}{2}}$ (this is only followed by s-p metals, not by transition metals).

5) Josephson predicted [3] macroscopic quantum effects called Josephson effects. In the DC Josephson effect, the total tunneling current $I$ across a SIS (superconductorinsulator-superconductor) junction would be determined by the change in phase of the order parameter across the junction. That leads to an important coherent behavior of the junction $I=I_{0} \frac{\sin \left(\pi \Phi / \Phi_{0}\right)}{\pi \Phi / \Phi_{0}}$ where $\Phi$ is the total magnetic flux in the junction, $\Phi_{0}$ is the flux quantum $h c / 2 e$, and $I_{0}$ depends on the temperature and structure of the junction.

Many physical properties of a superconductor can be simply explained by using Maxwell's equations and the two fluid model yielding, for example, the London equations. The two fluid model treats a superconductor as a perfect conductor with magnetic permeability $\mu=1$ and dielectric constant $\epsilon=1$. One assumes that there are two kinds of current density in a superconductor, the normal electron current density $\mathbf{j}_{\mathbf{n}}$ and the supercurrent density $\mathbf{j}_{\mathbf{s}}$. Then the total current density will be $\mathbf{j}$ $=\mathbf{j}_{\mathbf{n}}+\mathbf{j}_{\mathbf{s}}$. In this case, Maxwell's equations are:

$$
\begin{array}{r}
\nabla \cdot \mathbf{E}=\frac{4 \pi}{c} \rho \\
\nabla \times \mathbf{E}=-\frac{1}{c} \frac{\partial \mathbf{B}}{\partial \mathbf{t}} \\
\nabla \cdot \mathbf{B}=\mathbf{0}
\end{array}
$$




$$
\nabla \times \mathbf{H}=\frac{4 \pi}{c}\left(\mathbf{j}_{n}+\mathbf{j}_{s}\right)+\frac{1}{c} \frac{\partial \mathbf{E}}{\partial t}
$$

and the continuity equation is

$$
\frac{\partial \rho}{\partial t}+\nabla \cdot\left(\mathbf{j} n+\mathbf{j}_{s}\right)=0
$$

The normal current should satisfy Ohm's law

$$
\mathrm{j}_{n}=\sigma_{n} \mathbf{E} .
$$

The supercurrent velocity $\mathbf{v}_{\mathbf{s}}$ must satisfy Newton's second law

$$
m \frac{d \mathbf{v}_{s}}{d t}=-e \mathbf{E}
$$

The supercurrent density can be written:

$$
\mathbf{j}_{s}=-n_{s} e \mathbf{v}_{s}
$$

where $n_{s}$ is the number density of superconducting electrons. Using the following relation for the time derivative

$$
\frac{d \mathbf{v}_{s}}{d t}=\frac{\partial \mathbf{v}_{s}}{\partial t}+\mathbf{v}_{s} \cdot \nabla \mathbf{v}_{s}
$$

and by omitting the nonlinear terms and combining Eq. (1.7) - (1.9), we get

$$
\frac{\partial \mathbf{j}_{\mathrm{s}}}{\partial \mathrm{t}}=\frac{n_{s} e^{2}}{m} \mathbf{E} .
$$

This is called the first London equation. By substituting this equation into Maxwell equation (1.2) we get

$$
\nabla \times \mathbf{j}_{s}=-\frac{n_{s} e^{2}}{m c} \mathbf{B}
$$


This is called London's second equation. If we let $\alpha^{2}=\frac{n_{s} e^{2}}{4 \pi m c}$ and assume $\mathbf{j} s$ is independent of time, use Maxwell equation (1.4) Eq. (1.11) and consider the $\mathrm{j}_{n}=0$ case, one gets

$$
\nabla^{2} \mathbf{B}-\alpha^{2} \mathbf{B}=\mathbf{0} .
$$

By assuming that $B=B(z)$, we get $\frac{d^{2} \mathbf{B}}{d^{2} z}-\alpha^{2} \mathbf{B}=0$. The solution for that equation is $\mathbf{B}=\mathbf{B}_{0} \exp (-\alpha z)$. This says that the magnetic field cannot penetrate into the superconductor, except for a surface layer. The thickness of this layer $\frac{1}{\alpha}$ is called the London penetration depth $\lambda_{l}$.

There are two weak points in this model. $\lambda_{l}$ is not dependent on external field, and $\lambda_{l}$ is not dependent on the size of the sample. In order to overcome these two weaknesses, Ginzburg-Landau theory was introduced.

\section{Ginzburg-Landau Theory for Superconductivity}

\section{Landau Theory}

Landau theory [4] for second-order phase transitions said that the Gibbs free energy near $\mathrm{T}_{c}$ can be expanded in a power series in the order parameter $\psi$,

$$
G(T, P, \psi)=G_{o}(T, P)+\alpha(P)\left(T-T_{c}\right) \psi^{2}+C \psi^{4}
$$

The equilibrium value of the order parameter can be obtained by minimizing the free energy with respect to $\psi: \psi=\sqrt{\frac{\alpha\left(T_{c}-T\right)}{2 C}}$ for $T<T_{c}$ and $\psi=0$ for $T>T_{c}$. Then

$$
\begin{aligned}
G & =G_{o}-\frac{\alpha^{2}}{4 C}\left(T_{c}-T\right)^{2}, T>T_{c} \\
& =G_{o}, T>T_{c} .
\end{aligned}
$$


According to the definition of entropy $S=-\left(\frac{\partial G}{\partial T}\right)$, one obtains $S=S_{o}\left(T>T_{c}\right)$ and $S=S_{o}-\frac{\alpha^{2}}{2 C}\left(T_{c}-T\right)\left(\mathrm{T}<\mathrm{T}_{c}\right)$. From thermodynamic theory we also get an expression for the specific heat $C_{p}$

$$
\begin{aligned}
C_{p}=T\left(\frac{\partial S}{\partial T}\right)_{p} & =C_{o}(T), T>T_{c} \\
& =C_{o}(T)+\frac{\alpha^{2} T}{2 C}, T<T_{c}
\end{aligned}
$$

By subtracting these two formulae Landau theory predicts a discontinuity in specific heat at $T_{c}$ given by

$$
\left(C_{p}\right)_{s}-\left(C_{p}\right)_{n}=\frac{\alpha^{2} T_{c}}{2 C}
$$

In the presence of a magnetic field $H$, the free energy becomes $G^{\prime}=G-H \psi$. By minimizing $G^{\prime}$ respect to $\psi$ and neglecting the $\psi^{3}$ term, one obtains $H=2 \alpha\left(T-T_{c}\right) \psi$. From the definition of susceptibility, $\chi=\frac{\psi}{H}$, we get

$$
\chi=\frac{1}{2 \alpha\left(T-T_{c}\right)} .
$$

\section{Ginzburg-Landau Theory for Superconductivity}

In order to extend Landau theory to the superconducting phase transition, Ginzburg and Landau [5] argued that the order parameter $\psi(r)$ corresponds to the superconducting wave function and therefore its square should be normalized to the density $n_{s}$ of superconducting electrons. That is, $\psi^{*}(\mathbf{r}) \psi(\mathbf{r})=n_{s}(\mathbf{r})$, where $\mathbf{r}$ is the position. Ginzburg-Landau theory assumes that the free energy in the superconducting state $G_{s}$ is

$$
G(\mathbf{r})_{s}=G_{n}-\alpha^{\prime}|\psi|^{2}+(1 / 2) \beta|\psi|^{4}+\frac{1}{2 m^{*}}\left|\left(-i \hbar \nabla-\frac{q^{*} A}{c}\right) \psi\right|^{2}-\int_{0}^{B_{a}} M \cdot d B_{a}
$$


where $G_{n}$ i sthe normal state free energy, according to the BCS theory, a Cooper pair has $m^{*}=2 m, n_{s}^{*}=n_{s} / 2, e^{*}=2 e, A$ is the vector potential which is defined as

$$
\mathbf{B}=\nabla \times \mathbf{A},
$$

$\mathrm{c}$ is the speed of light and $\alpha^{\prime}=\alpha\left(T_{c}-T\right)$. The gradient term comes from an increase in energy caused by a spatial variation of the order parameter. The integral term represents the increase in the superconducting free energy caused by the expulsion of magnetic flux from the superconductor.

The total superconducting free energy is

$$
G_{s}=\int G_{s}(\mathbf{r}) d \mathbf{r}
$$

To minimize the free energy with respect to $\psi(\mathbf{r})$, one sets $\frac{\delta G_{s}}{\delta \psi}=0$, yielding

$$
\left[\frac{1}{2 m^{*}}\left(-i \hbar \nabla-\frac{q^{*} A}{c}\right)^{2}-\alpha^{\prime}+\beta|\psi|^{2}\right] \psi=0 \text {. }
$$

Also according to the usual quantum-mechanical definition,

$$
j_{s}(r)=q^{*} v_{\mathcal{S}}(\mathbf{r})=-\frac{i q^{*} \hbar}{2 m^{*}}\left[\psi^{*} \nabla \psi-\psi \nabla \psi^{*}\right]-\frac{q^{* 2}}{m^{2} c} \psi^{*} \psi A
$$

These are called the first and second Ginzburg-Landau equations.

\section{Coherence Length}

Now let us study the first Ginzburg-Landau equation for $\mathbf{B}=0$, and $\mathbf{A}=\mathbf{0}$. If $\psi$ only depends on $z$, this equation becomes:

$$
-\frac{\hbar^{2}}{2 m^{*}} \frac{d^{2} \psi}{d z^{2}}-\alpha^{\prime} \psi+\beta|\psi|^{2} \psi=0
$$


If we assume that a real solution for $\psi$ exists where $\psi$ does not vary in space for $\mathrm{z} \rightarrow$ $\pm \infty$, we get $\psi_{\infty}^{2}=\alpha^{\prime} / \beta$. Defining $f=\frac{\psi}{\psi_{\infty}}$, the previous equation becomes

$$
-\frac{\hbar^{2}}{2 m^{*} \alpha^{\prime}} \frac{d^{2} f}{d z^{2}}-f+f^{3}=0
$$

Let $\xi=\sqrt{\frac{\hbar^{2}}{2 m^{*} \alpha^{\prime}}}$. Then

$$
-\xi^{2} \frac{d^{2} f}{d z^{2}}-f+f^{3}=0 .
$$

The solution is $f=\tanh \left(\frac{z}{\sqrt{2} \xi}\right)$, the famous soliton solution [6].

If there is only one pair of electrons in the system, then its wave function is thus $\psi=\tanh (z / \sqrt{2} \xi)$. The size $\xi$ of this Cooper pair is called the coherence length, and is a measure of the minimum distance over which the superpair wavefunction can decrease to zero.

If we consider dynamic processes, then we should add a time term into Eq. (1.27). The simplest way is to add a second derivative term:

$$
\frac{d^{2} f}{d x^{2}}-\frac{d^{2} f}{d \tau^{2}}+f-f^{3}=0
$$

where $x=z / \xi$ and $\tau=V t / \xi$ and $V$ is the velocity of the soliton. It is a very famous nonlinear differential equation. It has a soliton solution:

$$
f=\tanh \left[\frac{x-\tau}{\sqrt{2}}\right]
$$

The properties of this solution are as follows: 1) It does not change shape when it travels; 2) After two solitons go though each other their shapes do not change; 3) The interaction between solitons is not linear. For example when two solitons meet 
each other the principle of superposition does not hold. This leads to the possibility of big fluctuations, which the BCS theory does not address. 


\section{CHAPTER 2. FARADAY MAGNETOMETER}

\section{Introduction}

The Faraday magnetometer [7] has been widely used in many areas of scientific research, e.g., in biology.[8], chemistry [9], and especially in physics [10]-[17]. In many cases the Faraday magnetometer has proved to be not only useful but also essential, for example in measuring the magnetic susceptibility under pressure [10] [9], combined magnetic susceptibility and thermogravimetric analysis [11] [12], high magnetic field and high temperature measurements [13], and fast flux magnetization relaxation measurements in superconductors [14].

The research we are interested in requires using a magnetometer to measure the superconducting and normal state properties of high $T_{c}$ superconductors. Determining the normal state properties is crucial in order to establish a basis to understand the mechanism of high temperature superconductivity. The exchange interaction strength between the $\mathrm{Cu}$ spins of cuprates is about $1500 \mathrm{~K}$. Therefore one needs to characterize the high temperature $(>>300 \mathrm{~K})$ magnetic properties. In addition, $H_{c 2}$ $(T=0)$ for most high $T_{c}$ materials is above $10 \mathrm{~T}$. The Faraday magnetometer we installed is capable of temperatures to $1000 \mathrm{~K}$ and magnetic fields up to 8 Tesla.

The Faraday magnetometer technique is based on measuring the force on a magnetic dipole moment $\vec{m}$ in an external inhomogeneous magnetic field. Consider 
a general case with

$$
\begin{aligned}
& \vec{m}=m_{x} \vec{i}+m_{y} \vec{j}+m_{z} \vec{k} \\
& \vec{B}=B_{x} \vec{i}+B_{y} \vec{j}+B_{z} \vec{k},
\end{aligned}
$$

where $\vec{B}$ is the magnetic field at the position of the sample. The potential energy $U$ of $\vec{m}$ is

$$
U=-\vec{m} \cdot \vec{B},
$$

and the force on $\vec{m}$ is

$$
\vec{F}=-\nabla U .
$$

Inserting Eq. (2.3) into (2.4) and expanding in Cartesian coordinates, we get

$$
\begin{aligned}
\vec{F}=\frac{\partial\left(m_{x} B_{x}+m_{y} B_{y}+m_{z} B_{z}\right)}{\partial x} & \vec{i}+\frac{\partial\left(m_{x} B_{x}+m_{y} B_{y}+m_{z} B_{z}\right)}{\partial y} \vec{j} \\
& +\frac{\partial\left(m_{x} B_{x}+m_{y} B_{y}+m_{z} B_{z}\right)}{\partial z} \vec{k}
\end{aligned}
$$

In our case only the vertical $(z)$ component of the force can be detected and we assume the magnetic dipole moment to be homogeneous over the sample volume, so that

$$
F_{z}=m_{x} \frac{\partial}{\partial z} B_{x}+m_{y} \frac{\partial}{\partial z} B_{y}+m_{z} \frac{\partial}{\partial z} B_{z} .
$$

In the Faraday technique, the magnetic induction $\vec{B}$ is designed to vary only in the $z$ direction across the sample volume. Therefore, we neglect the first two terms in the previous equation and the force becomes

$$
F_{z}=m_{z} \frac{\partial}{\partial z} B_{z}
$$

A sketch of the Oxford Instruments Faraday magnetometer is shown in Fig. 2.1. 
1. Liquid N2

3. Gradient coil I

4. Gradient coil II

5. Main magnet Coil

6. He cooling space, variable temperature insert

7. Oven

8. Sample and sample holder

9. Quartz fiber

10. To variable temperature insert vacuum line

11. To sample space vacuum line

12. Loop to attach hangdown fiber

13. Electronic balance

14. Tare weight

15. Sample transfer window

16. Balance cover

17. Cryogen vacuum

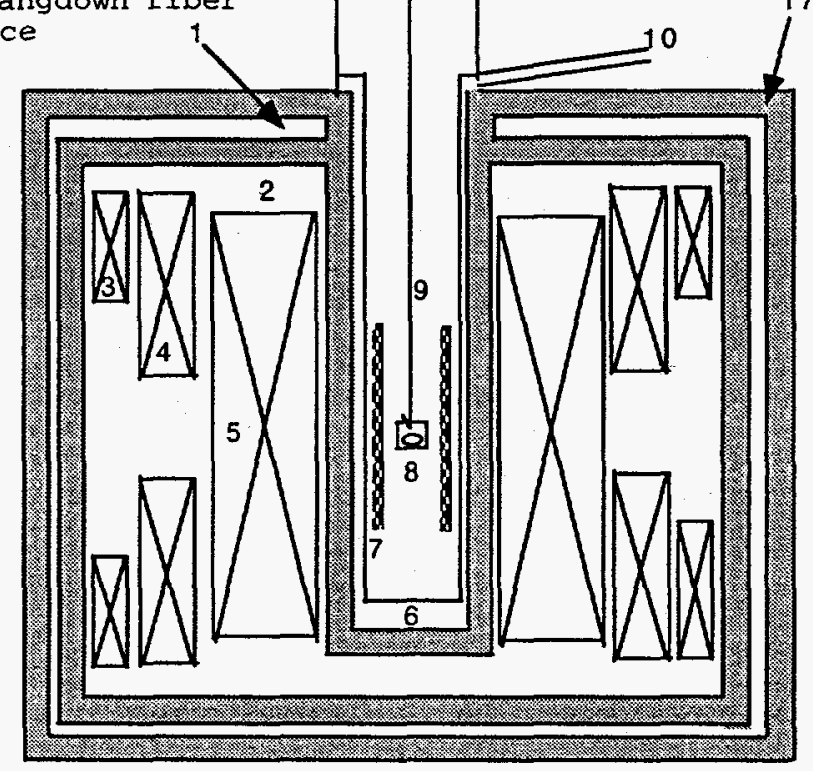

Figure 2.1: Sketch of the Oxford Instruments Faraday magnetometer 


\section{Sensitivity}

The Faraday method is one of the oldest ways for measuring static magnetization. Modern vacuum microbalances and superconducting magnets make it possible for the Faraday magnetometer to compete with SQUID magnetometers in terms of sensitivity. The Oxford Faraday magnetometer uses a Sartorius electronic vacuum microbalance with resolution $\Delta m=0.1 \mu \mathrm{g}$ and maximum capacity $3 \mathrm{~g}$, a superconducting solenoid with a field of up to $8 \mathrm{~T}$ and a superconducting quadrupole-type coil [18] enabling field gradients of up to $30 \mathrm{~T} / \mathrm{m}$ to be generated. For spins $1 / 2$ a $\mathrm{B} / \mathrm{T}$ ratio of $3 \mathrm{~T} / \mathrm{K}$ is needed to saturate the spins within $1 \%$ at $2 \mathrm{~K}$, and the $8 \mathrm{~T}$ field available with the Oxford Instruments Faraday magnetometer satisfies that criterion.

The sensitivity of the system is easily computed. Assume that the lowest magnetic moment that can be detected is $\mathrm{M}_{z}$. From Eq. (2.7),

$$
M_{z}=\frac{F_{z}}{\frac{d B}{d z}}
$$

Since $F_{z}=(\Delta m) \mathrm{g}$, where $\mathrm{g}=980.5 \mathrm{~cm} / \mathrm{s}^{2}$, one has

$$
M_{z}=\frac{\Delta m \mathrm{~g}}{\frac{d B}{d z}}
$$

Inserting the minimum detectable $\Delta m=0.1 \mu \mathrm{g}$ and maximum field gradient $\frac{d B}{d z}$ $=3000 \mathrm{G} / \mathrm{cm}$ into Eq. (2.9) yields the resolution in magnetization $M_{z}=3 \times$ $10^{-8} \mathrm{~cm}^{3} \mathrm{G}$. In units of Bohr magnetons $\mu_{B}$,

$$
M_{z}=\frac{3 \times 10^{-8} \mathrm{~cm}^{3} G}{\left(9.27 \times 10^{-21} \mathrm{~cm}^{3} G / \mu_{B}\right)}=3 \times 10^{12} \mu_{B},
$$

which corresponds to $3 \times 10^{12}$ spin $1 / 2$ impurity ions with saturated moments of $1 \mu_{B}$. If we consider a matrix of $\mathrm{Pt}$ with atomic weight $\mathrm{W}=196 \mathrm{~g} / \mathrm{mole}$, we have 
$\frac{6.023 \times 10^{23}}{196}=3.07 \times 10^{21} \mathrm{Pt}$ atoms in one gram of $\mathrm{Pt}$. The ratio of the minimum detectable number of impurity spins to $\mathrm{Pt}$ atoms in $1 \mathrm{~g}$ of $\mathrm{Pt}$ is therefore

$$
\frac{3 \times 10^{12}}{3.07 \times 10^{21}}=1 \times 10^{-9} \text {. }
$$

This ratio corresponds to 0.001 at. $\mathrm{ppm}$ of saturated spin $1 / 2$ ions in a $\mathrm{Pt}$ matrix. The Quantum Design SQUID magnetometer has a magnetic moment sensitivity of $10^{-8} \mathrm{~cm}^{3} \mathrm{G}$. Thus our Faraday magnetometer has comparable sensitivity $\left(3 \times 10^{-8}\right.$ $\mathrm{cm}^{3} \mathrm{G}$ ). In addition this system is capable of operation from $1.5 \mathrm{~K}$ to $1000 \mathrm{~K}$ and with applied magnetic fields from $0 \mathrm{~T}$ to $8 \mathrm{~T}$, which exceed the corresponding ranges ( 1.5 $\mathrm{K}$ to $400 \mathrm{~K} ; 0$ to $5.5 \mathrm{~T}$ ) of our SQUID magnetometer. These enhanced ranges justify the extra effort to run this system compared to a commercial SQUID magnetometer.

\section{Pumping Station}

The Faraday magnetometer is composed of three main parts; first an electronic cabinet accommodating power supplies, temperature controllers, and a data acquisition system; second a cryogenic system and the balance; third a vacuum control unit, including pumping facilities, vent valves and vacuum gauges.

The pumping station part of the Faraday magnetometer is homemade here in Iowa State University and Ames Laboratory. The author considered available pump facilities, the needs of the system, and availability of commercial pumping stations, and optimized the system according to these factors. Due to the special requirements of the Faraday magnetometer, we needed to design a special multipurpose vacuum pumping station, and at the same time to get an optimal plan in terms of both budget 
and function of the station.

There are two special components of this pumping station. First, the high vacuum part requires a vacuum of $10^{-6}$ to $10^{-8}$ torr, with a pumping speed of roughly $80 \mathrm{l} / \mathrm{s}$ at $10^{-4}$ torr. Second, the helium pumping system is high throughput to allow the vapor pressure and hence the temperature of the liquid helium in a large bath cryostat to be reduced below 1 atmosphere $(4.2 \mathrm{~K})$. The pumping speed should be about $700 \mathrm{l} / \mathrm{min}$ at pressures between 0.1 torr (corresponding to about $1 \mathrm{~K}$ ) and 760 torr (corresponding to $4.2 \mathrm{~K}$ ) [21].

The available Duo-Seal 1398 mechanical pump has a pumping speed of 1500 $1 /$ min at a pressure between $10^{-1}$ torr and 1 atmosphere, which is more than the required speed $700 \mathrm{l} / \mathrm{min}(11.7 \mathrm{l} / \mathrm{s})$ in the same pressure range. However there is a long distance between the pump and our Faraday magnetometer, so we need to consider the conductance between the pump and the Faraday balance. The pumping speed is defined as the volume of gas per unit of time $\frac{d V}{d t}$ which the pumping device removes from the system at the pressure existing at the inlet to the pump.[19] The pumping speed $S_{F}$ at the Faraday magnetometer, connected by a conductance $C$ to a pump having a pumping speed $S_{P}$, is given by

$$
\frac{1}{S_{F}}=\frac{1}{S_{P}}+\frac{1}{C}
$$

When conductances are connected in series, the total conductance $C_{t}$ is given by

$$
\frac{1}{C_{t}}=\frac{1}{C_{1}}+\frac{1}{C_{2}}+\frac{1}{C_{3}}+\ldots
$$

For the mechanical pump the pumping speed is almost constant between $10^{-1}$ and 760 torr. The conductance $C$ on the other hand is proportional to the pressure in most of this pressure range (viscous flow range), but approaches a constant as the 
pressure reaches about $10^{-1}$ torr or less (molecular flow range). Therefore if the lowest conductance (molecular flow range) meets our requirements then the pumping speed will be sufficient over the entire operating pressure range. Therefore we use the molecular flow conductance to estimate the mimimum pumping line diameter.

In the molecular flow limit the conductance for a long tube of constant cross section follows the Knudsen formula [20]:

$$
C=3.81(T / M)^{1 / 2} D^{3} / L(\text { liter } / \mathrm{sec}),
$$

where $D(\mathrm{~cm})$ is the diameter of the tube, $L(\mathrm{~cm})$ is the length of the tube, $C($ liter $/ \mathrm{sec})$ is the conductance, $T$ is the absolute temperature, and $M$ is the molecular weight of the gas pumped in $\mathrm{g} / \mathrm{mole}$. At room temperature $T=293 \mathrm{~K}$ and for He gas $M=$ 4. This formula is independent of the pressure because in the Knudsen regime the molecules pass through the long tube without colliding with each other.

In the molecular flow range the conductance for an elbow is between [19]

$$
C=3.81(T / M)^{1 / 2} D^{3} /\left[L_{1}+L_{2}+1.33 D\right]
$$

and

$$
C=3.81(T / M)^{1 / 2} D^{3} /\left[L_{1}+L_{2}\right],
$$

where $L_{1}$ and $L_{2}$ are the arm lengths of this elbow. So when

$$
L_{1} \text { and } L_{2}>>1.33 \mathrm{D}
$$

we may omit the $1.33 D$ in Eq. (2.15), and use Eq. (2.14) with total tube length $L$ $=L_{1}+L_{2}$. In our case $L \approx 8$ meters, and we now need to know what pumping line diameter $(D)$ should be chosen to satisfy the pumping speed requirement. From Eq. 
(2.14) for He one finds $C=42.69(1 / \mathrm{s})$ for $D=4$ inches, and $C=5.33(1 / \mathrm{s})$ for $D$ $=2$ inches. If we use the Duo-Seal 1398 mechanical pump with $S_{P}=25(1 / \mathrm{s})$, then $S_{F}=15.77(1 / \mathrm{s})$ for $D=4$ inches, and $S_{F}=4.39(1 / \mathrm{s})$ for $D=2$ inches. Therefore $D=4$ inches will be a reasonable choice (see Fig. 2.2).

For the high vacuum pumping station $\left(1 \times 10^{-6}\right.$ torr $)$, diffusion pump stations and turbo-molecular pumping stations are available. The advantages of a diffusion pumping station are reliability and cost. But the disadvantages are a worse vacuum limit compared to the vacuum limit $\left(10^{-10}\right.$ torr $)$ that can be reached by a turbomolecular pump, the presence of oil vapor, and the necessity of a liquid nitrogen cold trap. For a turbo-molecular pump the time to pump from one atmosphere to $10^{-6}$ torr is much less than for a diffusion pump. So generally speaking a turbo-molecular pump has better performance than an oil diffusion pump. However the maximam magnetic field a turbo-molecular pumping station can stand is about $100 \mathrm{G}$. In order to match this condition we need to separate the pump from the Faraday magnetometer by at least 2 meters. This separation will reduce the effective pumping speed considerably. Therefore we decided to use a diffusion pump station. We compared the products from Varian, Balzers, Edwards, Alcatel, and Veeco, and chose the Veeco VR-3000 diffusion pump station. The layout of the pumping lines is schematically illustrated in Fig. 2.2.

Faraday Magnetometer Operation

\section{Introduction}

The operation of the Faraday magnetometer can be summarized into the following five steps. 


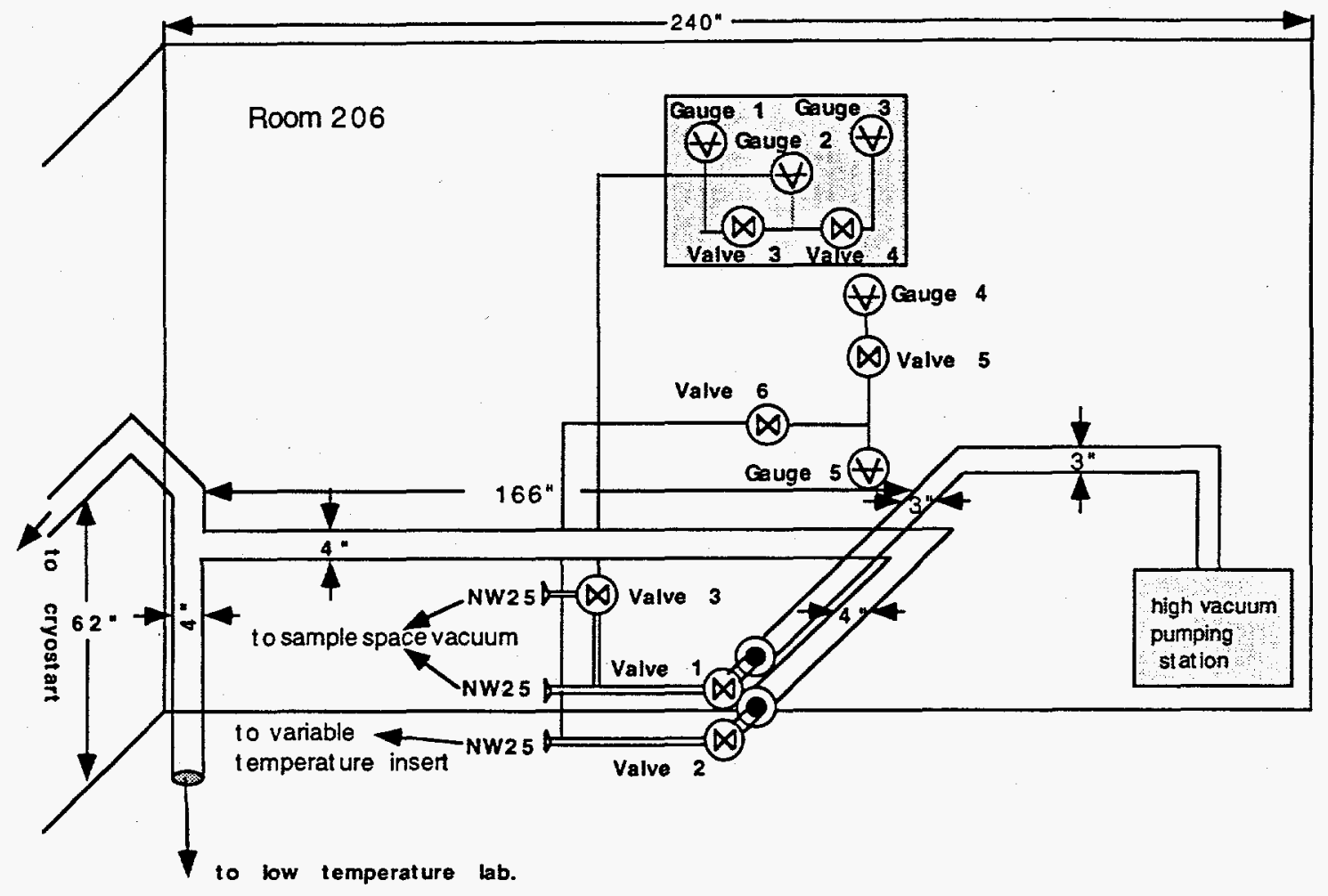

Figure 2.2: The pumping diagram for the Faraday magnetometer. The pump system in room A-206 consists of two separate pumping systems. The pump in the low temperature lab is connected to the variable temperature insert through valve 2 . The high vacuum pumping station in room A 206 is connected to the sample space through valves 1 and 3 . The pressure in the sample space is monitored by gauges 1,2 , and 3 . The pressure in the variable temperature insert space is monitored by gauges 4 and 5. 
1) The sample weight must be less than $3 \mathrm{~g}$, which is the maximum capacity of the microbalance. The diameter of the sample must be less than $1 / 2$ inch. The sample mass is also limited by the maximum force it will exert on the balance in the experimental magnetic field and magnetic field gradient; the maximum force should be $55 \mathrm{mg}$ for the $100 \mathrm{mg}$ range and $10 \mathrm{mg}$ for the $10 \mathrm{mg}$ range. The sample mass should be measured before and after each measurement. Balance drift due to buoyancy effects is not a factor in the magnetization measurements, since the method of taking the data corrects for balance drift (see below).

2) The sample and sample holder (if any) are suspended with a $221 / 4$ inch quartz fiber. The sample holder is usually a quartz bucket or a thin pure gold wire. The sample holder contribution should be premeasured and later subtracted from the raw data.

3) Extra care should be taken when loading the sample because of the delicate nature of the quartz fiber. The balance should be tared to read $0 \mathrm{mg}$ for the 10 $\mathrm{mg}$ range and $45 \mathrm{mg}$ for the $100 \mathrm{mg}$ range. The centering of the sample should be checked. The sample space should be evacuated by using the high vacuum pumping station to $10^{-6}$ torr for 12 hours, and refilled with He gas to the pressure required.

4) The data taking process is controlled by software provided by Oxford Instruments. The raw data should be reduced using software "convert.exe" (see below), and the calibration data file "zrwcalif.dat" (see below).

5) The Faraday magnetometer should be kept filled with liquid $\mathrm{N}_{2}$ and liquid He all the time that the magnetometer is operated.

The standard sample we used was the NIST pure Pt sample, with atomic weight $195.09 \mathrm{~g} / \mathrm{mole}$, density $21.54 \mathrm{~g} / \mathrm{cm}^{3}$, and molar volume $9.057 \mathrm{~cm}^{3} /$ mole. The cali- 
brations were done at room temperature. The range of gradient field was from 0 to $30 \mathrm{~T} / \mathrm{m}$, with a main field range from 0 to $8 \mathrm{~T}$. At room temperature $T=297 \mathrm{~K}$, . the susceptibility of $\mathrm{Pt}$ is $\chi(\mathrm{Pt})=0.991 \times 10^{-6} \mathrm{~cm}^{3} / \mathrm{g}$. The standard sample we measured has mass $196.57 \mathrm{mg}$. For this sample, $\chi=1.948 \times 10^{-7} \mathrm{~cm}^{3}$. Let the field equal to $1 \mathrm{~T}$ which is $10^{4} \mathrm{G}$, then the magnetic moment becomes $M=1.948 \times$ $10^{-3}\left(\mathrm{~cm}^{3} \mathrm{G}\right)$. Now if we apply a gradient field $\frac{d B}{d z}=10 \mathrm{~T} / \mathrm{m}=1000 \mathrm{G} / \mathrm{cm}$, then the force should be:

$$
\begin{aligned}
F(\text { dyne }) & =M\left(\mathrm{~cm}^{3} \mathrm{G}\right) \frac{d B}{d z}\left(\frac{\mathrm{G}}{\mathrm{cm}}\right)=1.946(\text { dyne }) \\
F(\text { newton }) & =M\left(\mathrm{~A} \mathrm{~m}^{2}\right) \frac{d B}{d z}\left(\frac{\mathrm{T}}{\mathrm{m}}\right)=1.946 \times 10^{-5}(\text { newton })
\end{aligned}
$$

Therefore by measuring the force on the sample at a known field gradient we can get the sample magnetic moment $M=F / \frac{d B}{d z}$.

\section{Magnetic Moment Measurement Sequence}

The measurement sequence is designed to take balance drift into account. The Oxford six point sequence is to first set the gradient $\frac{d B}{d z}$ to zero, measure the force at time $t_{1}, F\left(t_{1}\right)$, second measure the force at positive gradient $F\left(t_{2}\right)$, third measure the force at zero gradient again $F\left(t_{3}\right)$, fourth measure the force at the zero gradient $F\left(t_{4}\right)$, fifth measure the force at negative gradient $F\left(t_{5}\right)$, sixth measure the force at zero gradient field $F\left(t_{6}\right)$. The final formula for the force is:

$$
\begin{array}{r}
2 F=F\left(t_{2}\right)-\left[\frac{F\left(t_{3}\right)-F\left(t_{1}\right)}{t_{3}-t_{1}}\left(t_{2}-t_{1}\right)+F\left(t_{1}\right)\right] \\
-F\left(t_{5}\right)+\left[\left(\frac{F\left(t_{6}\right)-F\left(t_{4}\right)}{t_{6}-t_{4}}\left(t_{5}-t_{4}\right)+F\left(t_{4}\right)\right] .\right.
\end{array}
$$


The relationship between the gradient coil current in Amperes, $I_{G}$, and the field gradient $d B_{z} / d z$, is defined as

$$
\frac{d B_{z}}{d z}=\frac{I_{G}}{\text { calif }}
$$

where calif is a calibration factor which is $0.0341 \mathrm{~A} \mathrm{~cm} / \mathrm{G}$ at low fields.

For example, a data file for the Pt sample at $T=297 \mathrm{~K}$ is listed in Table 2.1. For these data, $H=70000 \mathrm{G}$ and

$$
\frac{d B}{d z}=\frac{33 \mathrm{~A}}{0.0341\left(\frac{\mathrm{A} \mathrm{cm}}{\mathrm{G}}\right)}=968 \frac{\mathrm{G}}{\mathrm{cm}} .
$$

From Table 2.1 and Eq. (2.19) we get $F=13.78$ dyne. From Eq. (2.8) the sample magnetic moment is then

$$
M=\frac{F}{\frac{d B}{d z}}=\frac{13.78 \text { dyne }}{967.7 \mathrm{G} / \mathrm{cm}}=1.423 \times 10^{-2} \mathrm{~cm}^{3} \mathrm{G}
$$

Therefore the sample susceptibility is

$$
\begin{gathered}
\chi\left(\mathrm{cm}^{3}\right)=\frac{M\left(\mathrm{~cm}^{3} \mathrm{G}\right)}{H(\mathrm{G})}=2.033 \times 10^{-7} \mathrm{~cm}^{3} \\
\chi\left(\mathrm{m}^{3}\right)=\frac{M\left(\mathrm{Am}^{2}\right)}{H(\mathrm{~A} / \mathrm{m})}=2.327 \times 10^{-12} \mathrm{~m}^{3}
\end{gathered}
$$

and gram susceptibility is

$$
\chi g=\frac{\chi}{M_{\mathrm{Pt}}}=1.034 \times 10^{-6} \frac{\mathrm{cm}^{3}}{\mathrm{~g}} .
$$

\section{Units}

The data file generated by the Oxford Instruments software gives a susceptibility in SI volume units. The relation between the molar susceptibility in CGS units and 
Table 2.1: A Faraday magnetometer raw data file for a Pt sample of mass 196.57 $\mathrm{mg}$, where $\mathrm{g}=980.5 \mathrm{~cm} / \mathrm{s}^{2}$

\begin{tabular}{llll}
\hline \hline Time (seconds) & Gradient Current (A) & Force/g (gram) & Magnetic field (T) \\
162222 & +0.0000 & 0.00051400 & 7.000 \\
162248 & +33.0000 & 0.01457300 & 7.000 \\
162272 & +0.0000 & -0.00051700 & 7.000 \\
162310 & +0.0000 & +0.00023000 & 7.000 \\
162334 & -33.0000 & -0.01381200 & 7.000 \\
162360 & +0.0000 & +0.00022700 & 7.000 \\
\hline \hline
\end{tabular}

Table 2.2: The Faraday magnetometer data file (for $\mathrm{H}=2000 \mathrm{G} \mathrm{G}=3 \mathrm{~A}$ ) for $\mathrm{Pt}$ with different units

\begin{tabular}{llll}
\hline \hline B external $(\mathrm{T})$ & 0.200000 & B external $(\mathrm{G})$ & 2000 \\
Gradient $(\mathrm{T} / \mathrm{m})$ & 0.880 & Gradient $(\mathrm{G} / \mathrm{cm})$ & 88.0 \\
H $(\mathrm{A} / \mathrm{m})$ & $1.592 \times 10^{5}$ & H (oersted) & 2000 \\
Temperature $(\mathrm{K})$ & 297.00 & Temperature $(\mathrm{K})$ & 297.00 \\
Force $(\mathrm{N})$ & $3.260 \times 10^{-7}$ & Force (dyne) & $3.260 \times 10^{-2}$ \\
$M($ volume) $(\mathrm{A} / \mathrm{m})$ & 40.60 & $M$ (volume) $(\mathrm{G})$ & 0.0406 \\
$\chi($ volume $)$ & $+2.54610^{-4}$ & $\chi$ (volume) & $2.027 .10^{-5}$ \\
\hline \hline
\end{tabular}


the volume susceptibility in SI units is

$$
\chi\left(\frac{\mathrm{cm}^{3}}{m o l e}\right)=\chi(\text { volume }) \frac{1}{4 \pi} V_{M}\left(\frac{\mathrm{cm}^{3}}{\mathrm{~mole}}\right),
$$

where $V_{M}$ is the molar volume. Therefore the volume susceptibility for $\mathrm{Pt}$ in SI units is

$$
\begin{aligned}
\chi & =\frac{4 \pi \chi\left(\frac{\mathrm{cm}^{3}}{\mathrm{~mole}_{\mathrm{e}}}\right)}{V_{M}\left(\frac{\mathrm{cm}^{3}}{\mathrm{~mole}_{\mathrm{e}}}\right)} \\
& =\frac{4 \pi\left[0.991 \times 10^{-6}\left(\mathrm{~cm}^{3} / \mathrm{g}\right)\right][195.09(\mathrm{~g} / \mathrm{mole})]}{9.057\left(\mathrm{~cm}^{3} / \text { mole }\right)} \\
& =2.682 \times 10^{-4}(\text { dimensionless })
\end{aligned}
$$

Table 2.2 gives examples of conversions between SI units and CGS units for various variables.

\section{Sample Holder Contribution and Demagnetization Factor}

For our standard sample we did not use any sample holder except the long quartz fiber. The error introduced by the long quartz fiber is less than $1 \%$ (discussed below).

For the Ta sample we used a quartz bucket, which will give us a relatively large signal, and we will discuss this case in this section. A magnetization versus magnetic field curve was measured for the sample holder along with the long quartz fiber. The $\frac{M}{H}=\chi$ versus $H$ plot shows an up-turn in the low field range, which can be fitted to

$$
\chi=\frac{c 1}{H-c 2}+\chi_{o}
$$

For the sample holder used with the Ta sample we get

$$
\chi=\frac{0.141 \times 10^{-8} \mathrm{G} \mathrm{cm}^{3}}{H+557(\mathrm{G})}-3.95 \times 10^{-8} \mathrm{~cm}^{3} .
$$


The Ta magnetic data were corrected with that expression.

Above, we omitted the influence of the demagnetization factor $N_{d}$ on the computed susceptibility. This is, we assumed that the magnetic field internal to the sample, $H$, is equal to $H_{a}$, where $H_{a}$ is applied magnetic field. In order to estimate the error associated with this approximation, we use the formula:

$$
H=H_{a}+N_{d} M
$$

In the case of Table 2.2, $M=3.592 \times 10^{-4}\left(\mathrm{~cm}^{3} \mathrm{G}\right)$, and the volume magnetization is

$$
M=3.592 \times 10^{-4}\left(\mathrm{~cm}^{3} \mathrm{G}\right) \frac{\text { density }}{\text { mass }}=0.040 \mathrm{G} .
$$

Therefore,

$$
H=H_{a}+N_{d} M=2000.16 \mathrm{G},
$$

where we assume $N_{d}=4 \pi / 3$. Therefore it is a good approximation to omit the demagetization factor correction in this case.

\section{Calibration of Gradient Magnetic Field}

The Faraday magnetometer has two superconducting coils, the main field solenoid and the gradient field coil. These two coils affect each other in the high field range, resulting in nonlinearity between the electric currents passed through the coils and the magnetic fields caused by those currents. The way that Oxford uses to correct this effect is to fit the gradient coil magnetic field gradient versus magnetic field applied by the main solenoid to a bipolynomial which is determined from calibration experiments. 
Now let us consider the gradient field calibration in detail. We need to determine the dependence of the calibration factor, calif, in Eq. (2.20) on magnetic field $H$ and magnetic gradient $G=d H / d z$. We assume the calibration factor is dependent on the field as well as the field gradient, so we need to calculate calif $\left(H, I_{G}\right)$ as a function of $H$ and $I_{G}$,

$$
\operatorname{calif}\left(H, I_{G}\right)=a+b H+c H^{2}+d H I_{G}+e I_{G}+f\left(I_{G}\right)^{2}
$$

where $a, b, c, d, e$, and $f$ are constant coefficients. However there is still an error associated with the fitting, and this error sometimes will reach as high as 5 to 10 percent depending on the range of $H$ we select to fit.

Therefore the author developed an alternative program named "convert.bas" which numerically calculates the values of $\operatorname{calif}\left(H, I_{G}\right)$ and stores them in a data file called "zrwcalif.cal". In order to explain the process we use the data file for the Pt sample of mass $196.57 \mathrm{mg}$ in Table 2.1 as an example where $H=70000 \mathrm{G}$ and $I_{G}$ $=33 \mathrm{~A}$. Using the standard (NIST) value for the susceptibility $\chi=0.991 \times 10^{-6}$ $\mathrm{cm}^{3} / \mathrm{g}$, one predicts

$$
M=\chi H=1.364 \times 10^{-2} \mathrm{~cm}^{3} \mathrm{G} .
$$

Using Eqs. (2.18) and (2.20), the calibration factor is found to be

$$
\text { calif }=\frac{I_{G} M}{F}=\frac{(33 \mathrm{~A})\left(1.364 \times 10^{-2} \mathrm{~cm}^{3} \mathrm{G}\right)}{13.77 \text { dyne }}=0.03268 \mathrm{~A} \mathrm{~cm} / \mathrm{G}
$$

instead of 0.0341 cited above. Using the same process for different $H$ and $I_{G}$, one obtains the array calif $\left(H, I_{G}\right)$. As noted above, the software stores the array in a calibration file called zrwcalif.cal. 
When the software analyzes the data for an arbitrary Faraday magnetometer experiment, it looks for the calibration factors from the calibration file stored previously in zrwcalif.cal. If the values of $H$ and $I_{G}$ do not match the values in the calibration data file zrwcalif.cal, the software calculates calif $\left(H, I_{G}\right)$ by using a linear interpolation formula, according to the values of the calibration factor at the four nearest locations where the calibration factors are known, $\operatorname{calif}\left(H 1, I_{G^{1}}\right)$, $\operatorname{calif}\left(H 1, I_{G^{2}}\right)$, $\operatorname{calif}\left(H 2, I_{G^{1}}\right), \operatorname{calif}\left(H 2, I_{G^{2}}\right)$ :

$$
\operatorname{calif}\left(H, I_{G}\right)=\operatorname{calif}\left(H, I_{G} 1\right)+\frac{\operatorname{calif}\left(H, I_{G}{ }^{2}\right)-\operatorname{calif}\left(H, I_{G}\right)^{1)}}{I_{G^{2}}-I_{G}}\left(I_{G}-I_{G}\right)
$$

where

$$
\begin{aligned}
& \operatorname{calif}\left(H, I_{G^{1}}\right)=\operatorname{calif}\left(H 1, I_{G} 1\right)+\frac{\operatorname{calif}\left(H 2, I_{G^{1}}\right)-\operatorname{calif}\left(H 1, I_{G}{ }^{1)}\right.}{H 2-H 1}(H-H 1) \\
& \operatorname{calif}\left(H, I_{G^{2}}\right)=\operatorname{calif}\left(H 1, I_{G^{2}}\right)+\frac{\operatorname{calif}\left(H 2, I_{G^{2}}\right)-\operatorname{calif}\left(H 1, I_{G^{2}}\right)}{H 2-H 1}(H-H 1)
\end{aligned}
$$

Therefore the the magnetization (CGS) of the sample is:

$$
M(H, G)=\frac{F(H, G)(\text { dyne })}{I_{G}(\mathrm{~A}) /\left[\operatorname{calif}\left(H, I_{G}\right)(\mathrm{A} \mathrm{cm} / \mathrm{G})\right]} .
$$

A calibration data file zrwcalif.cal was generated using a standard Pt sample from NIST. The obtained calibration factors are plotted versus $H$ and $I_{G}$ in Fig. 2.3. The software and the calibration file were tested against several different standard samples (Ta, $\mathrm{Al}, \mathrm{Pt}$, and $\mathrm{Pd}$ ). For the Ta sample, the maximum difference in susceptibility for different $\left(H, I_{G}\right)$ values from $(0.2 \mathrm{~T}, 3.41 \mathrm{~A} \mathrm{~m} / \mathrm{T})$ to $(6 \mathrm{~T}, 13.64 \mathrm{~A} \mathrm{~m} / \mathrm{T})$ is 8 percent if the software with a constant calibration factor is used, but is only is 1 percent if the zrwcalif.cal calibration file is used, as shown in Figs. 2.4 and 2.5 respectively. 


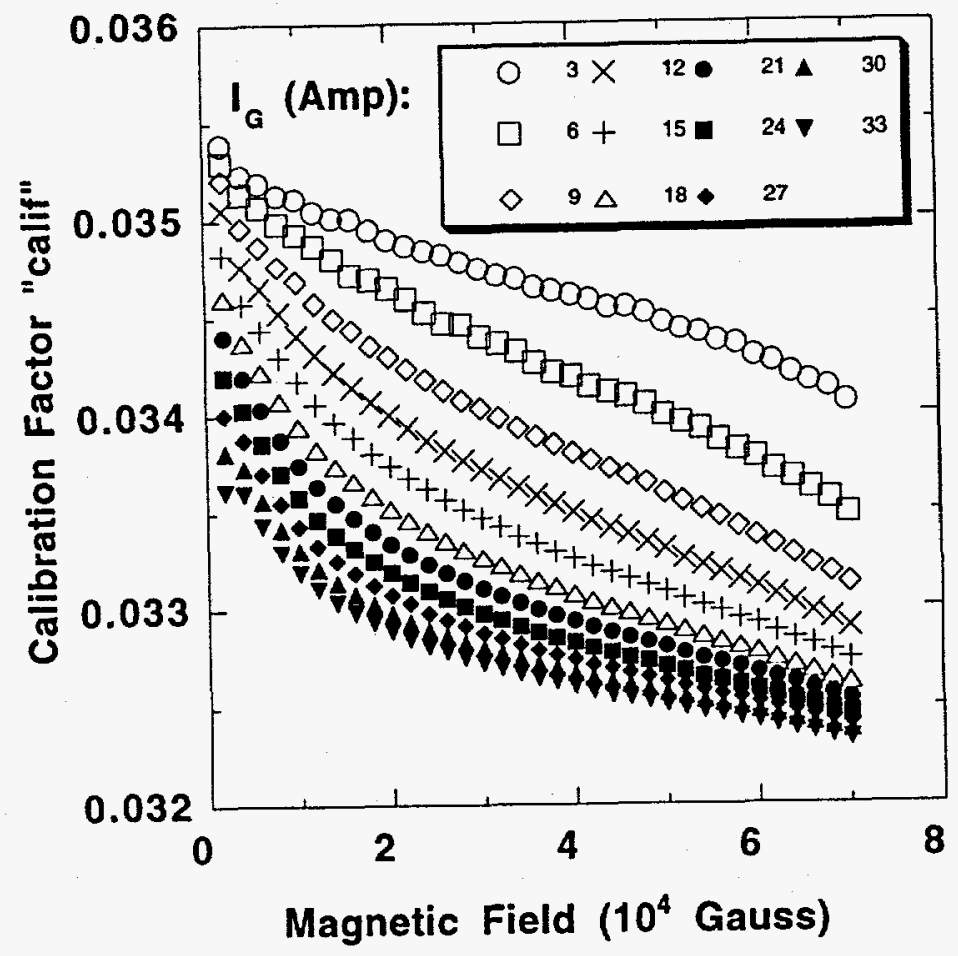

Figure 2.3: The calibration factor calif versus magnetic field at different gradient coil currents for the calibration data file zrwcalif.cal, where the symbols represent the value of gradient coil current $I_{G}$. 

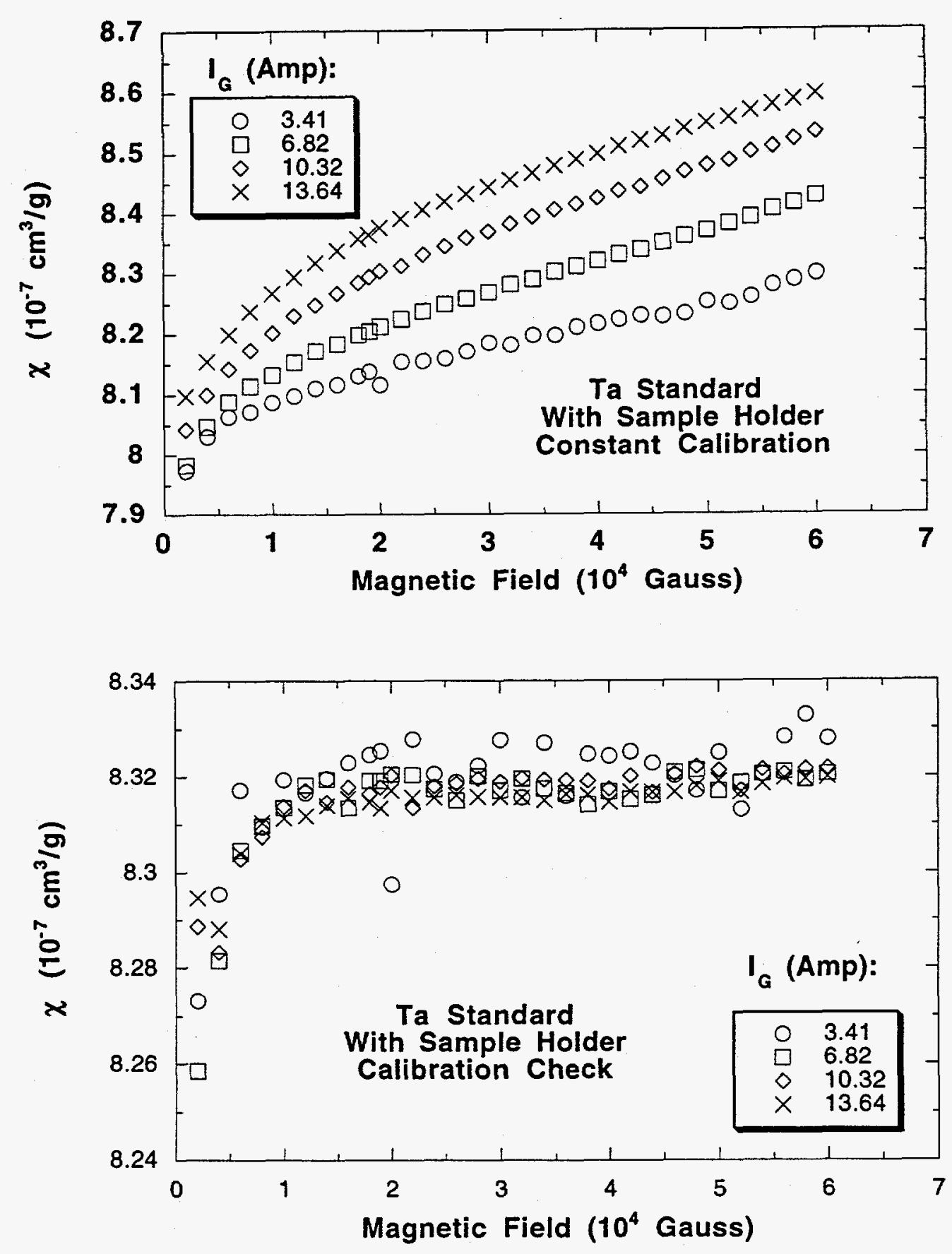

Figure 2.4: (a) The susceptibility determined according to constant calibration factor calif $=0.0341$ versus magnetic field at different gradient fields for a standard Ta sample and its sample holder. (b) The susceptibility, determined according to calibration file zrwcalif.cal, versus magnetic field at different gradient fields for a standard Ta sample and its sample holder. The symbols represent various values of the gradient coil current $I_{G}$. 

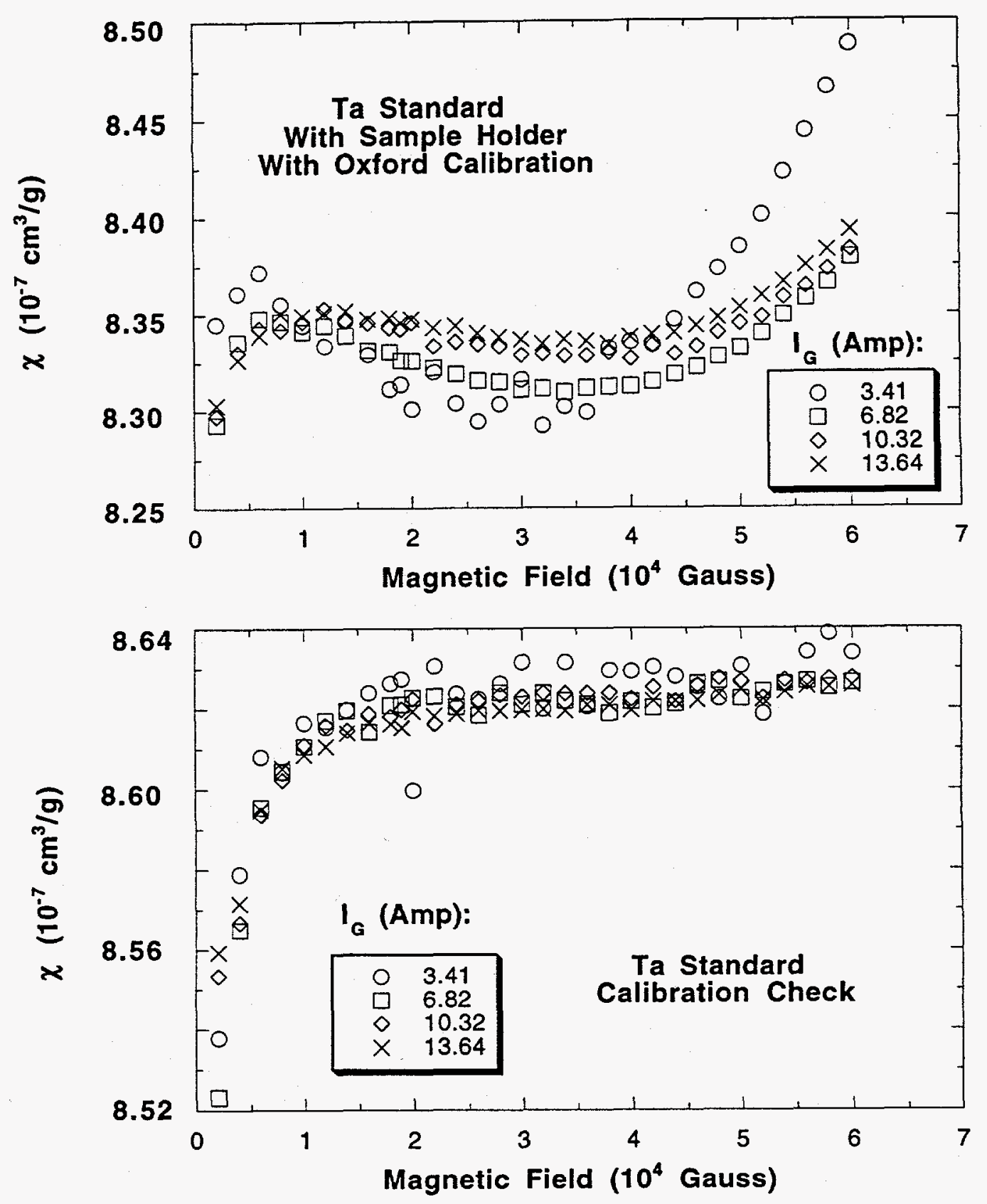

Figure 2.5: (a) The susceptibility determined according to a bipolynomial (Oxford Instruments' program) versus magnetic field at different gradient fields for a standard Ta sample and its sample holder. (b) The susceptibility, determined according to calibration file zrwcalif.cal, versus magnetic field at different gradient fields for a standard Ta sample corrected for the sample holder signal. The symbols represent the values of the gradient coil current $I_{G}$. 
Centering the Sample in the Magnetometer

Centering the sample was done by positioning the sample in zero field at a height such that the measured force difference $\Delta F_{+}$between positive magnetic gradient field and the zero gradient field, is equal to the force difference $\Delta F_{-}$between zero magnetic gradient field and negative gradient field. Thus, plots of $\Delta F_{+}$and $\Delta F_{-}$ versus sample height cross at the correct sample height. This centering assumes that the center of the main magnetic field is the same as the center of the gradient magnetic field.

In order to explain how the method works, let us examine a simple double gradient coil case. The expression for the magnetic field $B$ along the axis of a singleturn coil with current $I$ is

$$
B=\frac{\mu_{o} R^{2} I}{2\left(R^{2}+r_{o}^{2}\right)^{3 / 2}}
$$

where $R$ is the radius of the coil and $r_{0}$ is the distance from a point on the coil axis to the plane of the coil. In the case of $r_{o}=0$,

$$
B=\frac{\mu_{O} I}{2 R} \equiv B_{0}
$$

If we position two coils with opposite currents $a$ distance a apart, then the magnetic field on the axis of these two coils at the position $z$ of a sample on the axis is

$$
B=\frac{\mu_{0} I}{2 R}\left[\frac{1}{\left(1+\left(\frac{z}{R}+\frac{a}{2 R}\right)^{2}\right)^{3 / 2}}-\frac{1}{\left(1+\left(-\frac{z}{R}+\frac{a}{2 R}\right)^{2}\right)^{3 / 2}}\right],
$$

where $z$ is the distance between the center of these two coils and the sample. In the $\frac{z}{R} \ll 1$ case we may expand the denominators using Taylor series to yield

$$
B=B_{o} \frac{6 z}{\left(1+\frac{a}{2 R}\right)^{2} R}
$$


If the magnetic moment is proportional to the magnetic field then the force can be calculated in terms of the product of magnetic field and its gradient, which is proportional to

$$
B \times \frac{d B}{d z}=B_{o}^{2} \frac{6 z}{\left(1+\frac{a}{2 R}\right)^{4} R^{2}} .
$$

This expression is proportional to $z$. If we plot Weight(+gradient) - Weight(zero gradient) versus $z$ together with Weight(zero gradient) - Weight(-gradient) versus $z$, the crossing point of these two plots is the center $(z=0)$ of the two gradient coils.

\section{Temperature Calibration}

Temperature calibration of the Faraday magnetometer furnace is accomplished by first performing the calibration at several temperatures, then correcting the nominal temperature value read by the thermometer in the Faraday magnetometer.

A set of magnetic calibration standards (Perkin-Elmer No. 0219-0071) are used to perform the Curie temperature $T_{c}$ calibration on the Faraday magnetometer furnace. The standard samples we used are Alumel $\left(T_{c}=436 \mathrm{~K}\right)$, Nickel $\left(T_{c}=627 \mathrm{~K}\right)$, Nicoseal $\left(T_{c}=711 \mathrm{~K}\right)$, and Perkalloy $\left(T_{c}=869 \mathrm{~K}\right)$. The compositions of Nicoseal and Perkalloy are proprietary and would not be released by the manufacturer. The measurement results are shown in Figs. 2.6-2.10. 


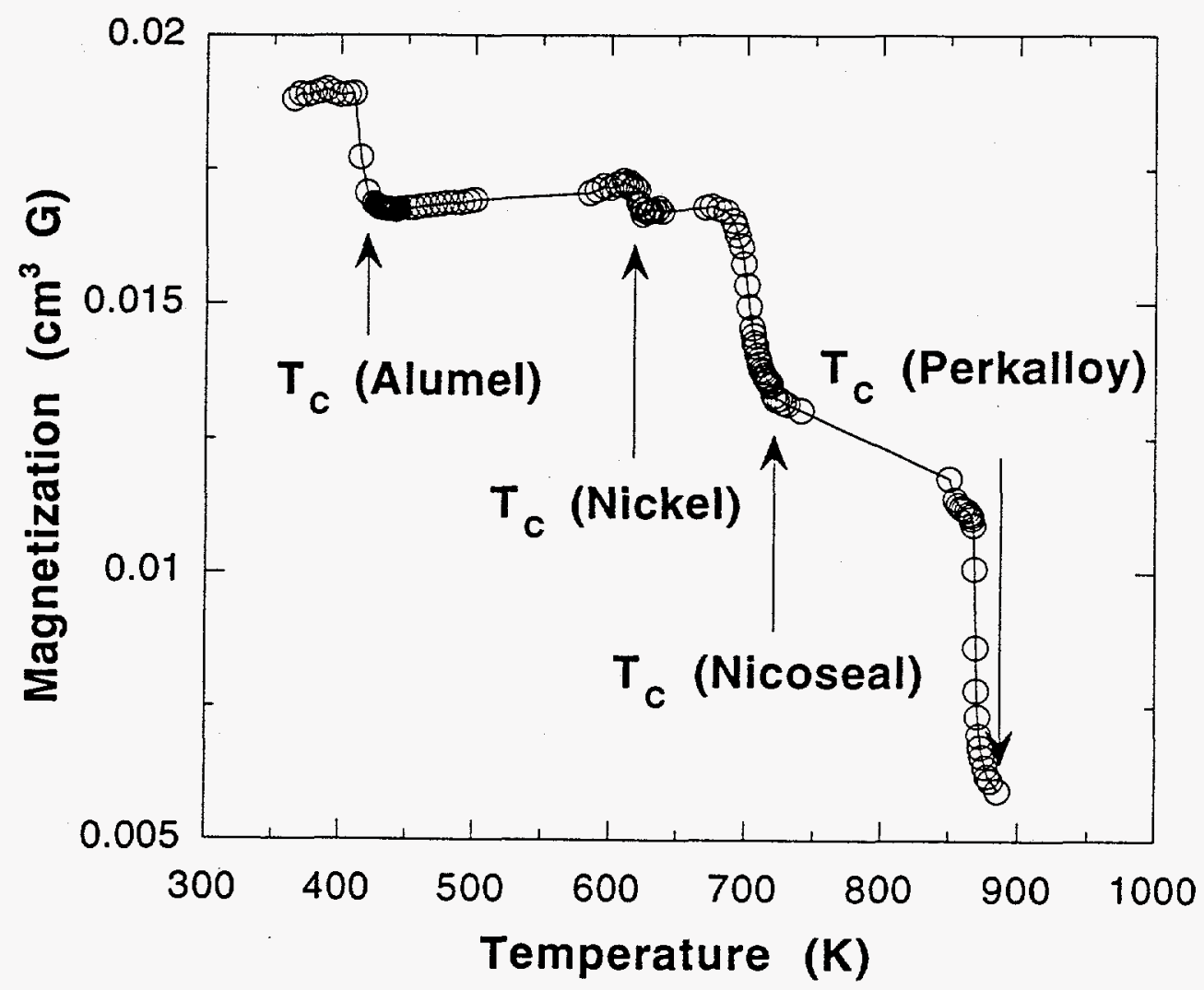

Figure 2.6: The magnetization versus temperature for four different standard calibration samples for the calibration of temperature 


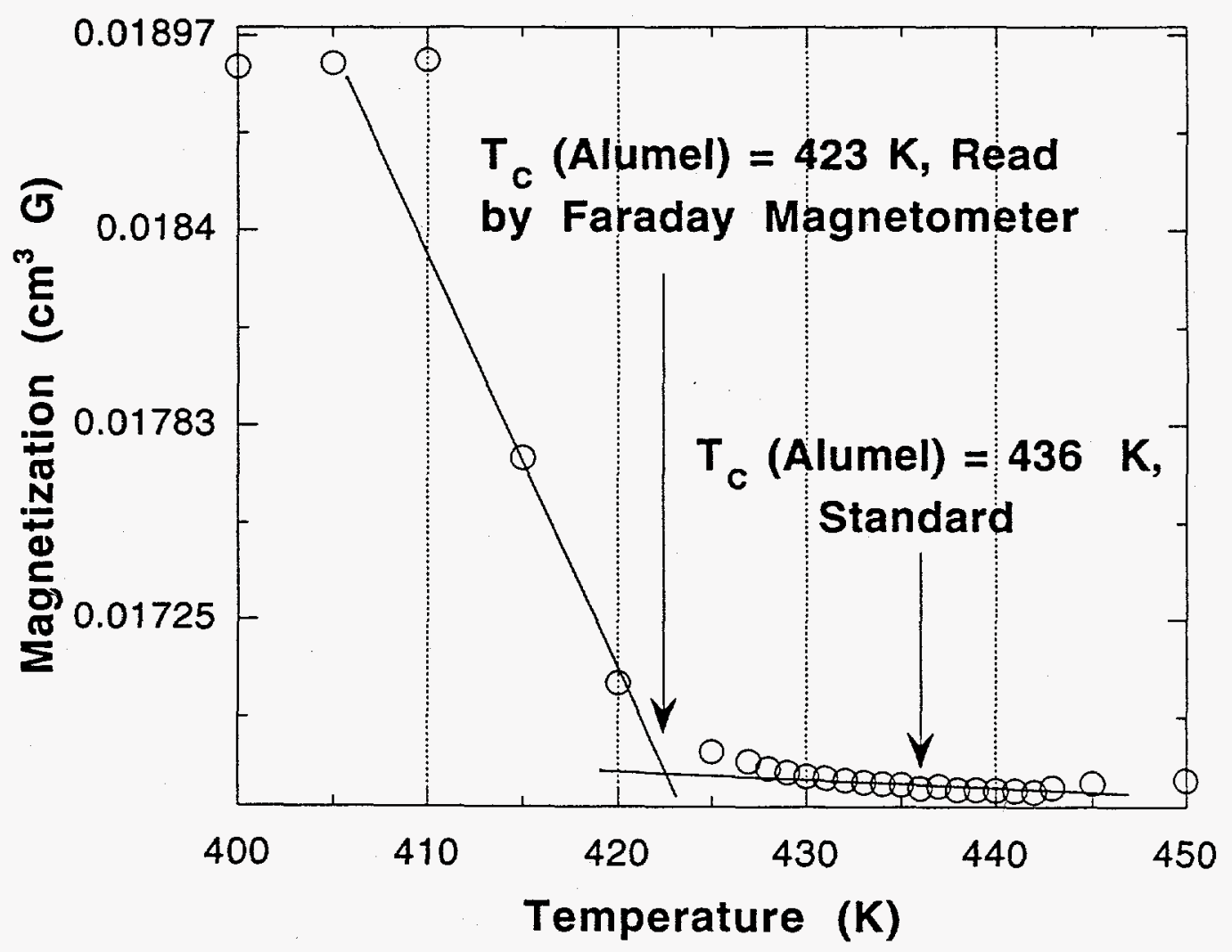

Figure 2.7: The magnetization versus temperature for a standard calibration sample of Alumel. 


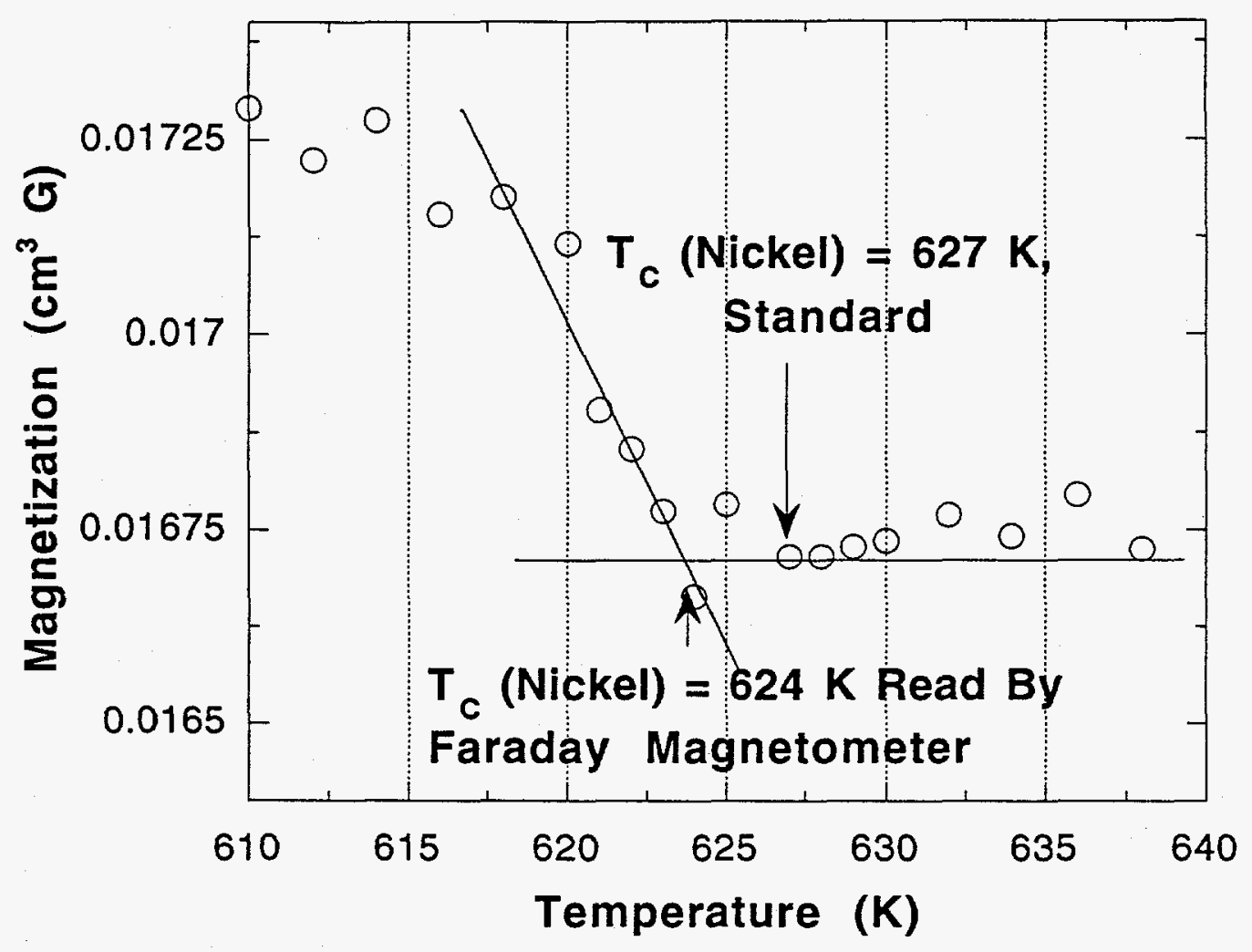

Figure 2.8: The magnetization versus temperature for a standard calibration sample of nickel. 


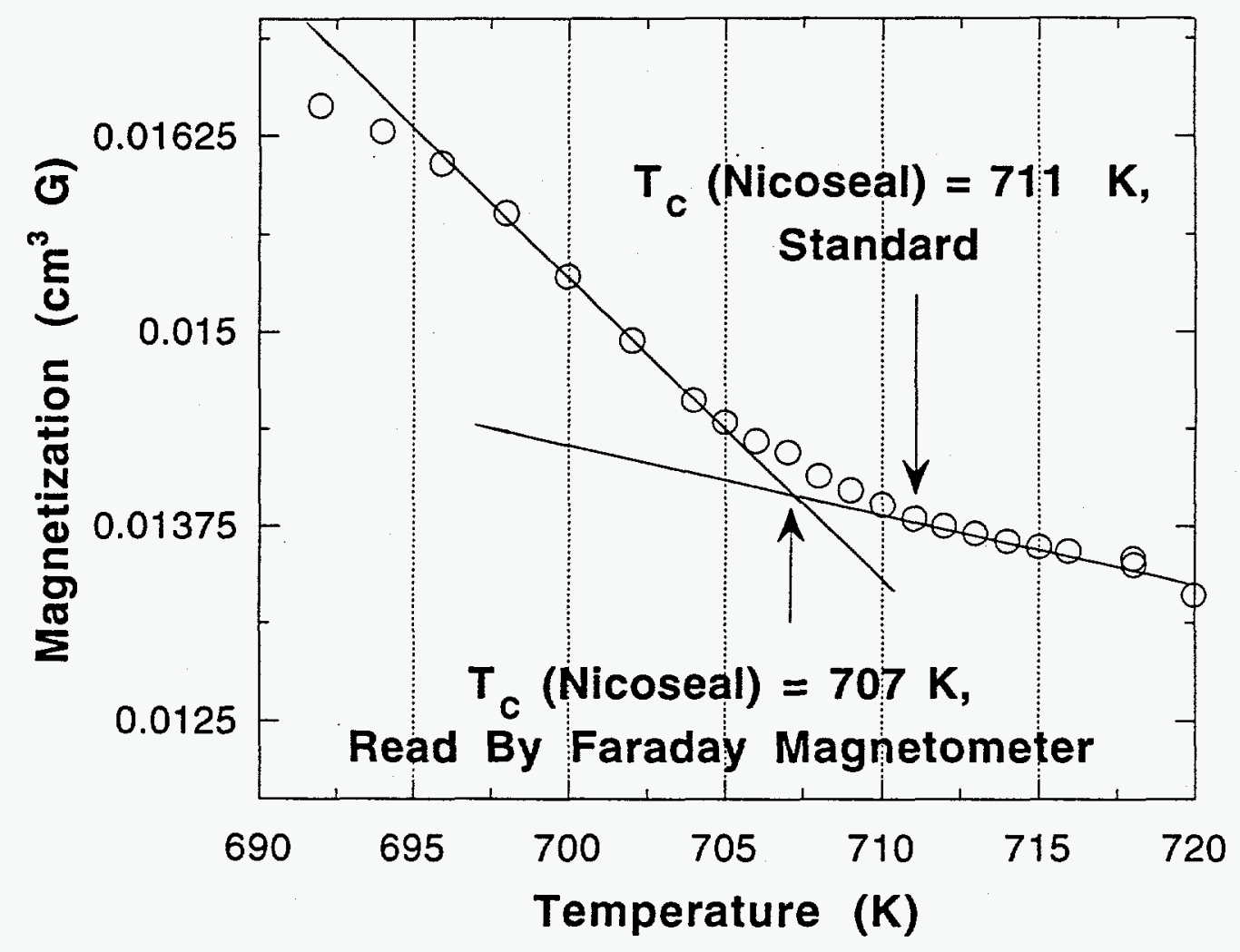

Figure 2.9: The magnetization versus temperature for a standard calibration sample of Nicoseal. 


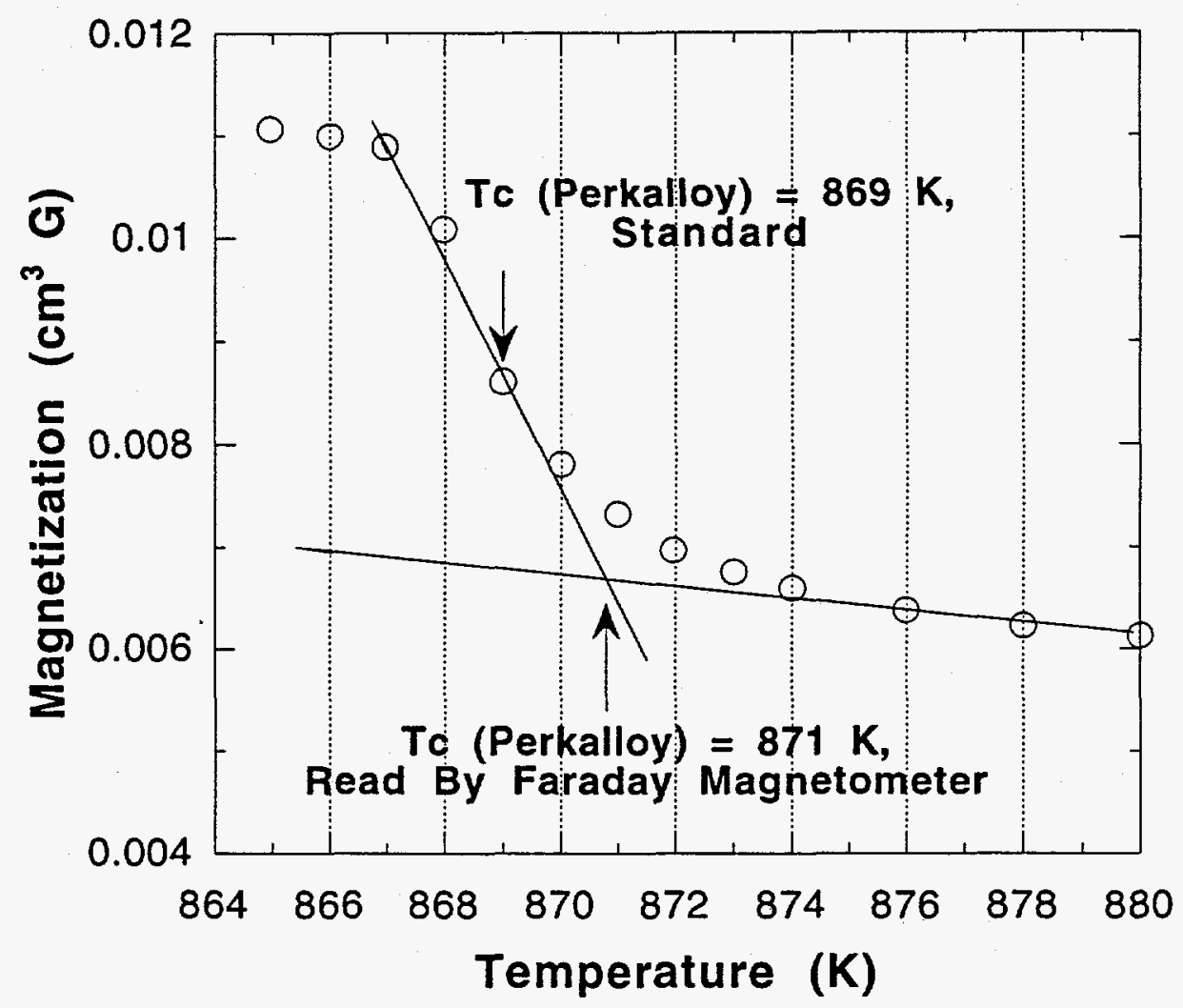

Figure 2.10: The magnetization versus temperature for a standard calibration sample of Perkalloy. 


\section{CHAPTER 3. SUPERCONDUCTING PROPERTIES OF $\mathrm{Ba}_{0.625} \mathrm{~K}_{0.375} \mathrm{BiO}_{3}$}

\section{Introduction}

Due to the unique composition [22], structure [23], and properties [24]-[26] of the superconductor $\mathrm{Ba}_{1-x} K_{x} \mathrm{BiO}_{3}$ system, further investigation of its properties is important to understand the mechanism of this high $T_{\mathcal{C}}$ superconductor. In order to understand more about the material, we systematically studied one of the fundamental parameters that characterizes superconductivity, the magnetic penetration depth. The magnetic penetration depth is related to many important properties of superconductors. For example the energy gap [27], and the effective mass and the superfluid density of a superconductor [25] are related to $\lambda(0)$. For ordinary superconductors at $T=0$, the superfluid density is identical to the carrier density for $T$ $\geq T_{c}$.

Two frequently used measurements to determine the magnetic penetration depth of a superconductor are magnetization and muon spin relaxation ( $\mu \mathrm{SR})$. Mitra et al. [28] obtained the temperature dependence of the penetration depth of $\mathrm{YBa}_{2} \mathrm{Cu}_{3} \mathrm{O}_{7 \pm y}$ and $\mathrm{Bi}_{2} \mathrm{Sr}_{2} \mathrm{CaCu}_{2} \mathrm{O}_{8}$ from magnetic measurements. Uemura et al. [29] used $\mu \mathrm{SR}$ to measure the temperature dependent penetration depth of a series of high $T_{c}$ materials. They also found a linear relation between $T_{c}$ and $\lambda(0)$ for "underdoped" high 
$T_{c}$ materials. Thus, it is interesting to see the results for the noncuprate high $T_{c}$ superconductor $\mathrm{Ba}_{1-x} K_{x} \mathrm{BiO}_{3}$ system.

In this chapter, the magnetic penetration depth, $\lambda(T)$, was measured by means of muon spin relaxation and the magnetization method for the same batch of single phase $B a_{0.675} K_{0.325} \mathrm{BiO}_{3}\left(T_{c}=26 \mathrm{~K}\right)$. The T-dependence of $\lambda$ for both methods indicates conventional s-wave pairing with the extrapolated (isotropic) value $\lambda(0)=$

$3400 \AA$, similar to that of $\mathrm{La}_{2}-x \mathrm{Sr}_{x} \mathrm{CuO}_{4}$ samples of the same $T_{c}[30]$. However as the temperature approaches $T_{c}$ there is a significant difference in magnetic penetration depth for these two methods, and a possible explanation for this difference will be discussed. The magnetization data were fitted using the Hao-Clem model [36]. The different results from these two methods can be explained by the field dependence of the penetration depth.

\section{Sample Preparation and Charactization}

The single phase material was made by the two-step method [23]. The starting materials were $\mathrm{BaO}(99.99 \%), \mathrm{KO}_{2}(95 \%)$, and $\mathrm{Bi}_{2} \mathrm{O}_{3}$ (99.9\%). The optimum synthesis conditions were determined by TGA, [24] SQUID magnetometry, and X-ray diffraction [23]. The conditions for making a $15 \mathrm{~g}$ batch of the sample were as follows. First, we rapidly $\left(1^{\circ} \mathrm{C} / \mathrm{min}\right)$ heated the sample in $\mathrm{N}_{2}$ to $700^{\circ} \mathrm{C}$ and maintained the sample at this temperature for one $\mathrm{hr}$. The sample was then rapidly cooled to room temperature by quenching in vacuum. Next, the sample was ground in a glove box for one $\mathrm{hr}$ in a $\mathrm{He}$ atmosphere and heated at $1{ }^{\circ} \mathrm{C} / \mathrm{min}$ in $\mathrm{O}_{2}$ to $550{ }^{\circ} \mathrm{C}$. This temperature was maintained for one $\mathrm{hr}$ and then the sample was oven-cooled to room temperature. $\mathrm{X}$-ray results showed very sharp diffraction peaks, and no impurity 
peaks were detected.

The magnetic susceptibility data were obtained with a Quantum Design Superconducting Quantum Interference Device (SQUID) magnetometer. We compared the penetration depth of the sample returned from the $\mu \mathrm{SR}$ measurements with the remainder of the sample (kept in the glove box). The results show that the penetration depth agreed to within $1 \%$ for the two samples.

From the $M$ vs. $H$ curve in the normal state at several different temperatures, we obtained the ferromagnetic impurity average spontaneous magnetization contribution, $M_{s}=4.14 \times 10^{-5} \mathrm{G} \mathrm{cm}^{3} / \mathrm{g}$. Also, by fitting the $M$ vs $T$ curve at $1 T$ near $T_{c}$ with a constant term plus an impurity Curie-Weiss contribution

$$
\chi(T)=\chi_{0}+\frac{C}{T-\theta}
$$

we obtained $\chi_{0}=-2.15 \times 10^{-7} \mathrm{~cm}^{3} / \mathrm{g}, C=2.36 \times 10^{-6} \mathrm{~cm}^{3} \mathrm{~K} / \mathrm{g}$, and $\theta=-10.6$ K. The magnetization data were corrected for these impurity contributions.

\section{Experimental Results}

\section{$\mu^{+}$SR Measurement}

The sample was made into a cylindrical pellet about $25 \mathrm{~mm}$ in diameter and 4 $\mathrm{mm}$ thick, which insured that all of the incident muons stopped in the sample. The $\mu \mathrm{SR}$ measurements were carried out by our collaborators using "surface" beams at the TRIUMF cyclotron. The apparatus and techniques are similar to those of previous work $[30],[31]-[35]$. Field cooled (FC) scans from the normal state were used for the determination of $\lambda(T)$, and zero-field-cooled (ZFC) measurements were carried out to test background and flux pinning effects for completeness. The asymmetry function 
in the transverse field (TF) consists of a precession signal (Larmor frequency for the muons in the average internal field) modulated by the spin relaxation function $G_{T F}$. In turn, $G_{T F}(t)$ is the Fourier transform of the lineshape due to the microscopic field distribution acting on the muon spin magnetic moment,

$$
G_{T F}(t)=\exp \left(-\sigma^{2} t^{2}\right)
$$

The Gaussian approximation (giving the second moment, $\sigma$, of the field distribution directly) yielded good fits to the data. Figure 3.1 shows the obtained $\sigma(T)$, compared for reference to the empirical relation

$$
\lambda(T)=\lambda(0)\left[1-\left(T / T_{c}\right)^{4}\right]^{-1 / 2}
$$

and Larmor frequency versus temperature. Thus, we obtain $T_{c}=(26 \pm 1) \mathrm{K}$ and a penetration depth $\lambda(0)=3400 \AA$ (see below), similar to that of $\mathrm{La}_{2-x} \mathrm{Sr}_{x} \mathrm{CuO}_{4}$ of comparable $T_{c}$.

\section{Magnetization Measurement}

The SQUID sample was put into a $3 \mathrm{~mm}$ I.D. quartz tube, which formed a cylinder $8 \mathrm{~mm}$ in length. The magnetization $M$ versus magnetic field $H$ data were averaged over increasing field and decreasing field, as an approximation to the reversible magnetization [28]. The zero field shielding at $5 \mathrm{~K}$ is equivalent to a $73 \%$ superconducting fraction. The $M(H)$ data are shown in Fig. 3.2. Additional $M(T)$ data are shown in Fig. 3.3. 

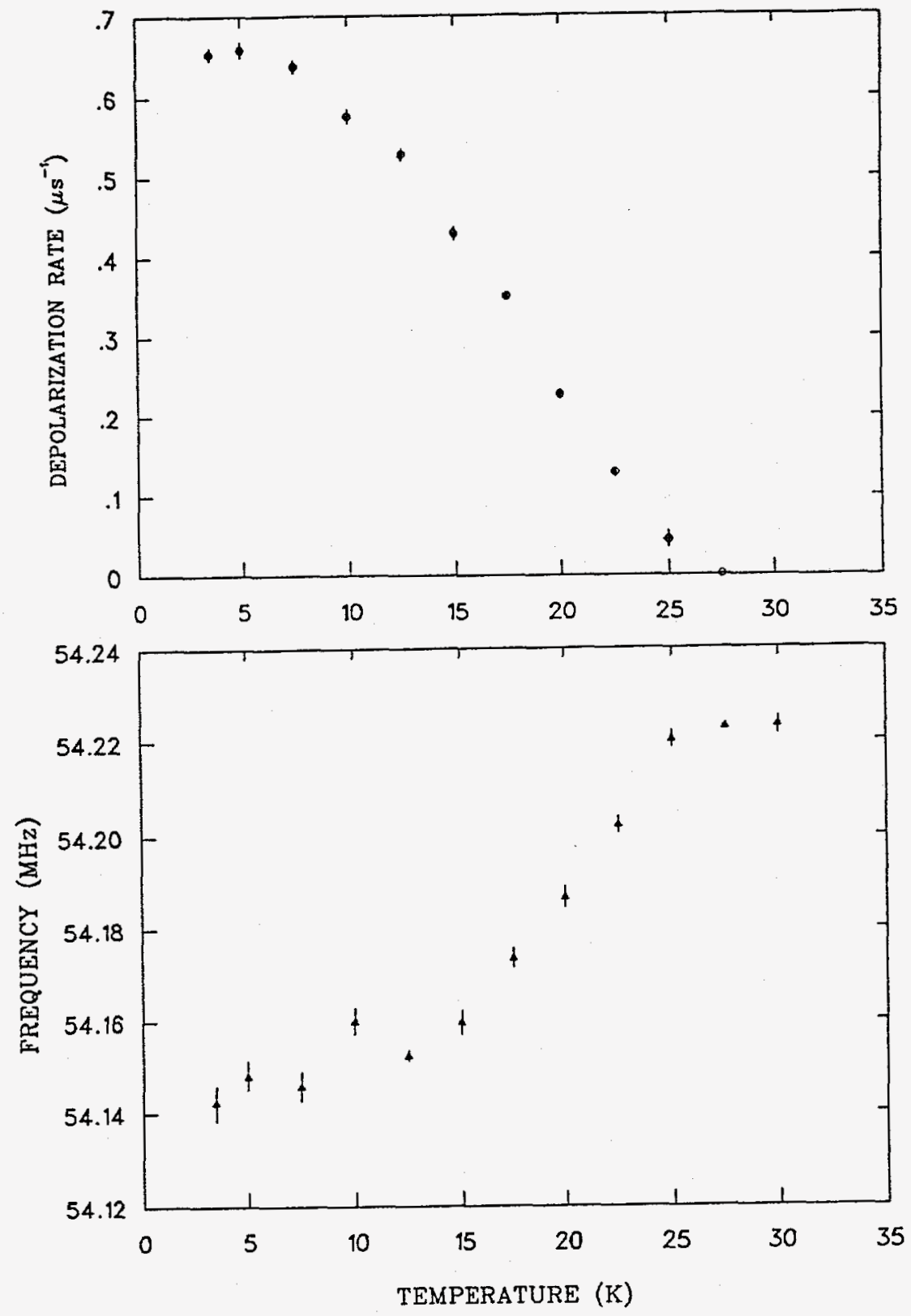

Figure 3.1: Temperature dependence of the Gaussian relaxation rate $\sigma$ and Larmor frequency for an applied field $H_{e x t}=4 \mathrm{kG}$ 


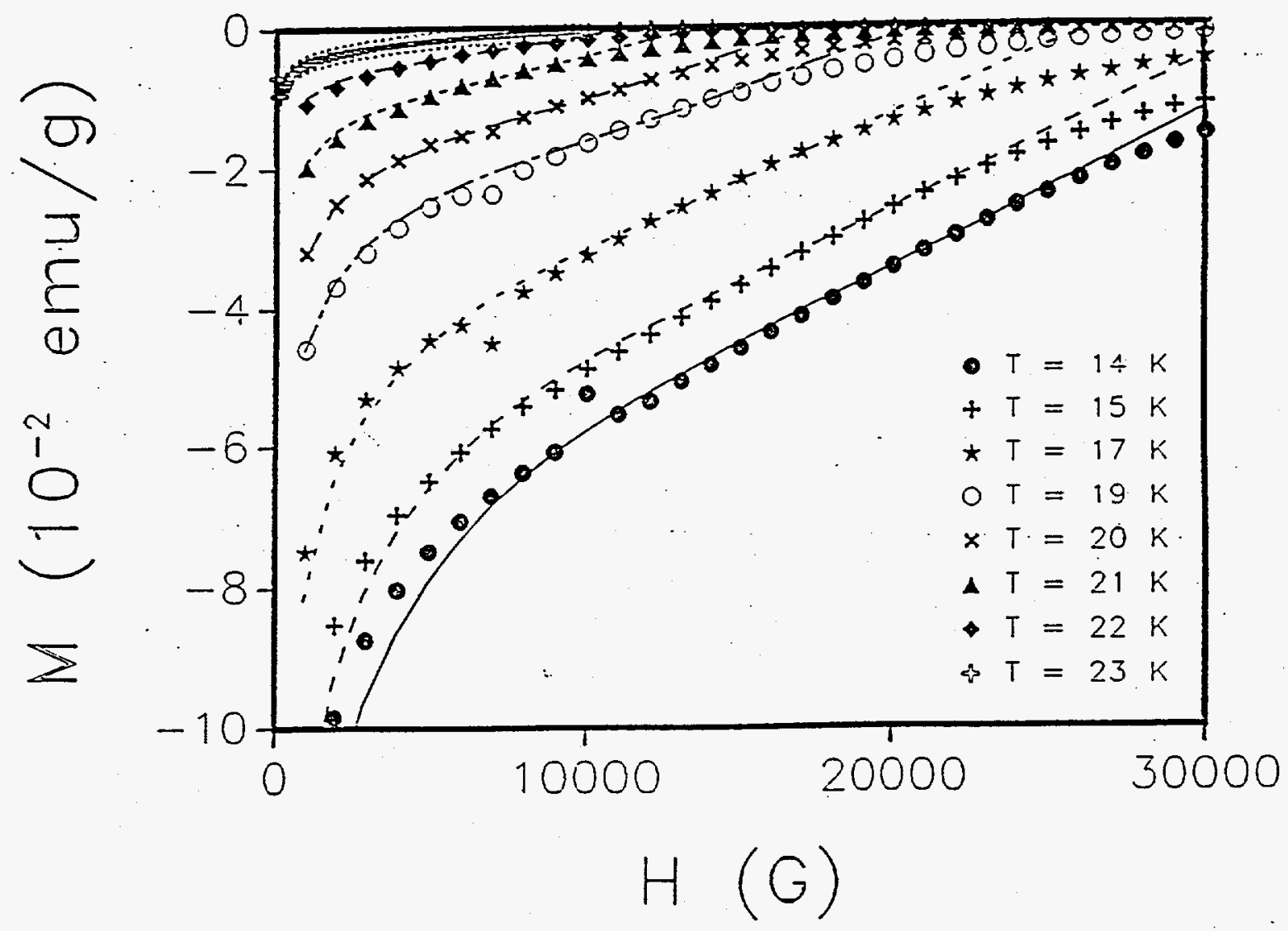

Figure 3.2: $\quad$ Magentization $M$ versus applied magnetic field $H$ at different temperatures $T$. The solid and dashed lines are the theoretical fits to the experimental points. 


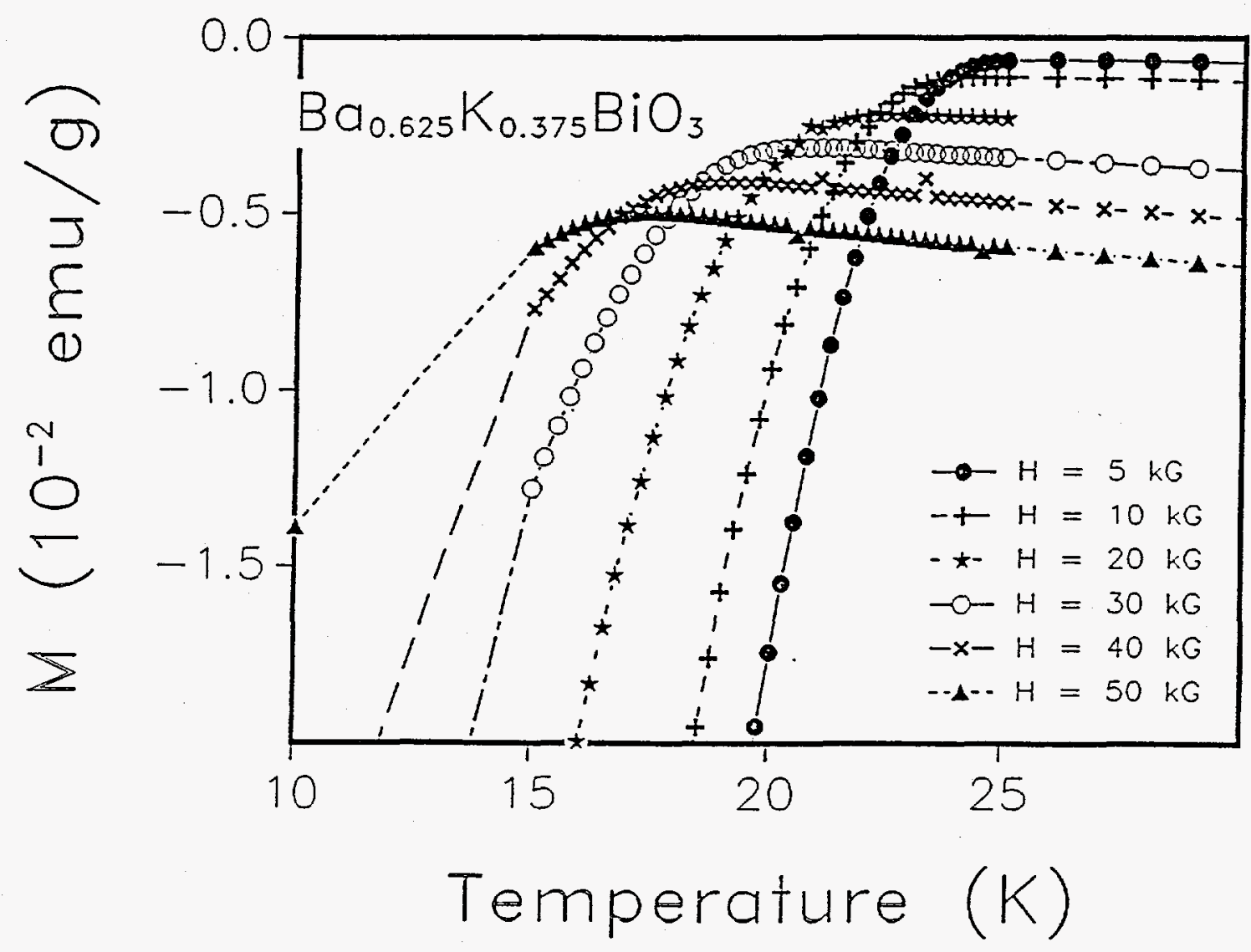

Figure 3.3: $\quad$ Magnetization $M$ versus Temperature $T$ at different magnetic fields $H$. 
Theory and Data Analysis

$\mu+\mathbf{S R}$

As detailed in the previous work on $\mathrm{YBa}_{2} \mathrm{Cu}_{3} \mathrm{O}_{7-x}$ and $\mathrm{La}_{2}-x \mathrm{Sr}_{x} \mathrm{CuO}_{4}$, [30][40] $\lambda$ is determined from the relaxation of the muon spin polarization signal in an external field $H_{\text {ext }}$ such that the separation between the vortices is much smaller than $\lambda$. Under this condition the $\mu^{+}$spin relaxation rate, $\sigma=1 / T_{2}$, is almost independent of $H_{e x t}$, and is given by the second moment of the microscopic field distribution [40], analogous to the field inhomogeneity broadening ( $T_{2}$ relaxation) in magnetic resonance [40]. From the relaxation time, $T_{2}$, the magnetic penetration depth is determined (assuming a perfect vortex lattice) by the relation, [38]

$$
\lambda=\sqrt{0.043 \phi_{0} \gamma_{\mu} T_{2}}
$$

where $\phi_{0}$ is the magnetic flux quantum, and $\gamma_{\mu}$ is the muon's gyromagnetic ratio.

\section{Reversible Magnetization and Penetration Depth}

Hao and Clem [36], starting from Ginzburg-Landau theory, obtained the following expression for the reversible magnetization $M$ :

$$
\begin{aligned}
-4 \pi M= & \frac{\kappa\left(1-\left(\frac{B}{\kappa}\right)^{4}\right) \xi_{v}^{2}}{2}\left[\frac{\left(\frac{B}{\kappa}\right)^{4}}{2} \ln \left(\frac{2}{B \kappa \xi_{v}^{2}}+1\right)-\frac{\left(\frac{B}{\kappa}\right)^{4}}{2+B \kappa \xi_{v}^{2}}+\frac{1-\left(\frac{B}{\kappa}\right)^{4}}{\left(2+B \kappa \xi_{v}^{2}\right)^{2}}\right] \\
& +\frac{\left[1-\left(\frac{B}{\kappa}\right)^{4}\right]\left(2+3 B \kappa \xi_{v}^{2}\right)}{2 \kappa\left(2+B \kappa \xi_{v}^{2}\right)^{3}}+\frac{1-\left(\frac{B}{\kappa}\right)^{4}}{2 \kappa \xi_{v} K_{1}\left(\kappa B \xi_{v}\right)} \\
& {\left[K_{0}\left(\xi v \sqrt{\left.1-\left(\frac{B}{\kappa}\right)^{4}+2 B \kappa\right)}-\frac{B \kappa \xi_{v} K_{1}\left(\xi_{v} \sqrt{\left.1-\left(\frac{B}{\kappa}\right)^{4}+2 B \kappa\right)}\right.}{\sqrt{1-\left(\frac{B}{\kappa}\right)^{4}+2 B \kappa}}\right]\right.}
\end{aligned}
$$


where $K_{n}(x)$ is a modified Bessel function of $n$th order,

$$
\begin{gathered}
\kappa \xi_{v 0}=\sqrt{2}\left[1-\frac{K_{0}^{2}\left(\xi_{v 0}\right)}{K_{1}^{2}\left(\xi_{v 0}\right)}\right]^{1 / 2}, \\
\left(\frac{\xi_{v}}{\xi_{v 0}}\right)^{2}=\left[1-2\left(1-\frac{B}{\kappa}\right)^{2} \frac{B}{\kappa}\right]\left[1+\left(\frac{B}{\kappa}\right)^{4}\right],
\end{gathered}
$$

and

$$
-4 \pi M=H-B
$$

The only adjustable parameters are $\kappa$ and $H_{C}$, where $\kappa$ is the Ginzburg-Landau parameter and $H_{C}$ is the thermodynamic critical field which is also a scaling parameter: $\sqrt{2} H_{c}=\kappa \phi_{0} / 2 \pi \lambda^{2}$, and $H_{c 2}(T)=\kappa \sqrt{2} H_{c}(T)$. We use $\sqrt{2} / \xi_{v 0}$ as an approximation for $\kappa\left(\xi_{v 0}\right)$, which results in a less than $1 \%$ error for $\kappa \geq 30$.

The $M$ vs $H$ data in Fig. 3.2 were fitted with the Hao-Clem theory [36]. The fitting parameters, $\kappa$ and $H_{c 2}$, were converted to $\lambda$ by the formula $\lambda^{2}=\kappa^{2} \phi_{0} /\left(2 \pi H_{c 2}\right)$, shown in Fig. 3.4. The upper critical fields from these fits, plotted in Fig. 3.5, are similar to results from the literature. [35]

\section{Discussion}

From the two fluid model, Eq. 3.3, we know that the penetration depth depends on the critical temperature $T_{c}$. However the critical temperature depends on the applied magnetic field, and in turn the penetration depth depends on the applied magnetic field. Therefore so we get a generalized two fluid model

$$
\lambda(T)=\lambda(0)\left[1-\left(\frac{T}{T_{c}(H)}\right)^{4}\right]^{-1 / 2} .
$$




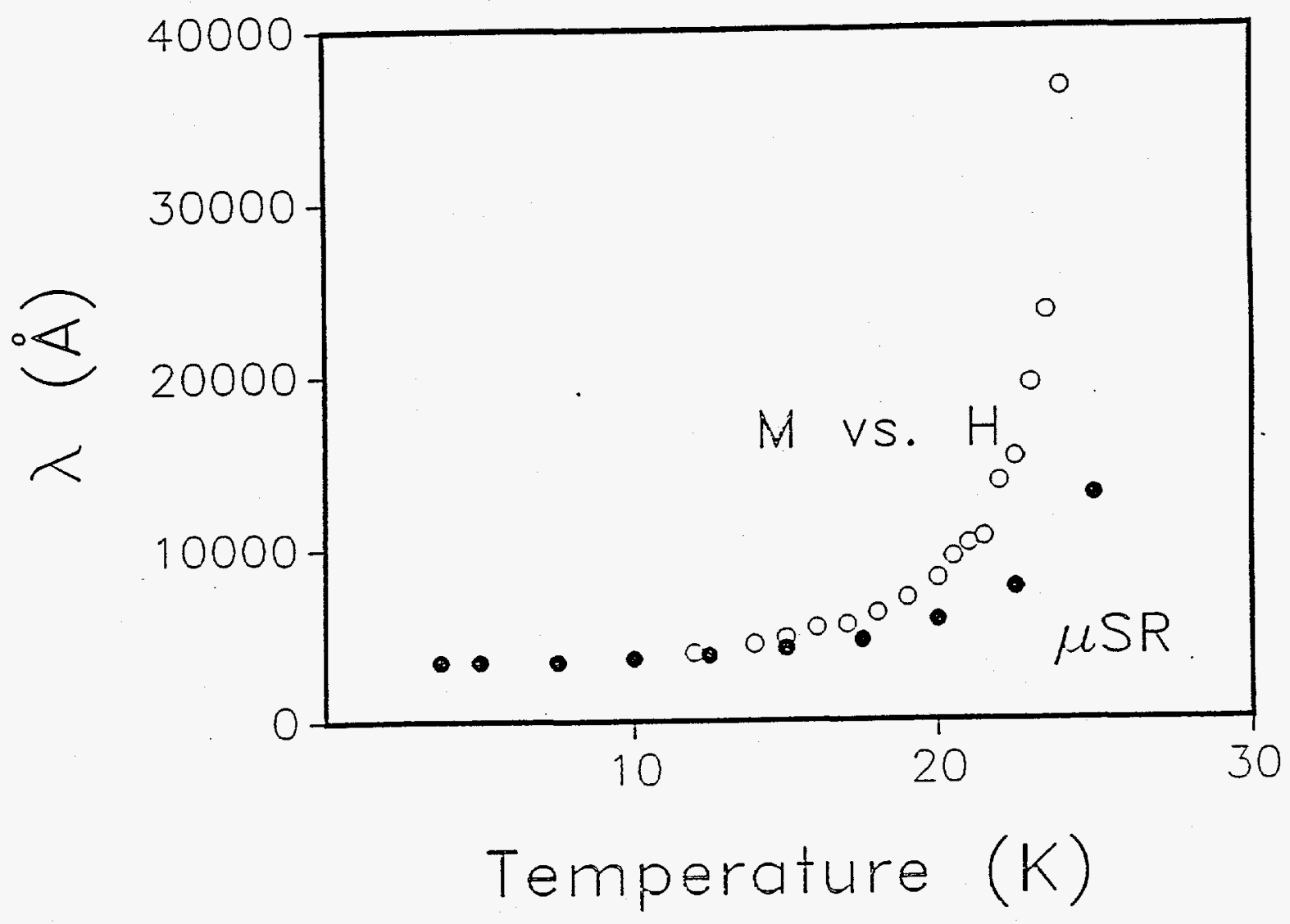

Figure 3.4: Penetration depth $\lambda$ versus temperature. The solid dots are $\mu \mathrm{SR}$ data, and the open circles are magnetization data. 


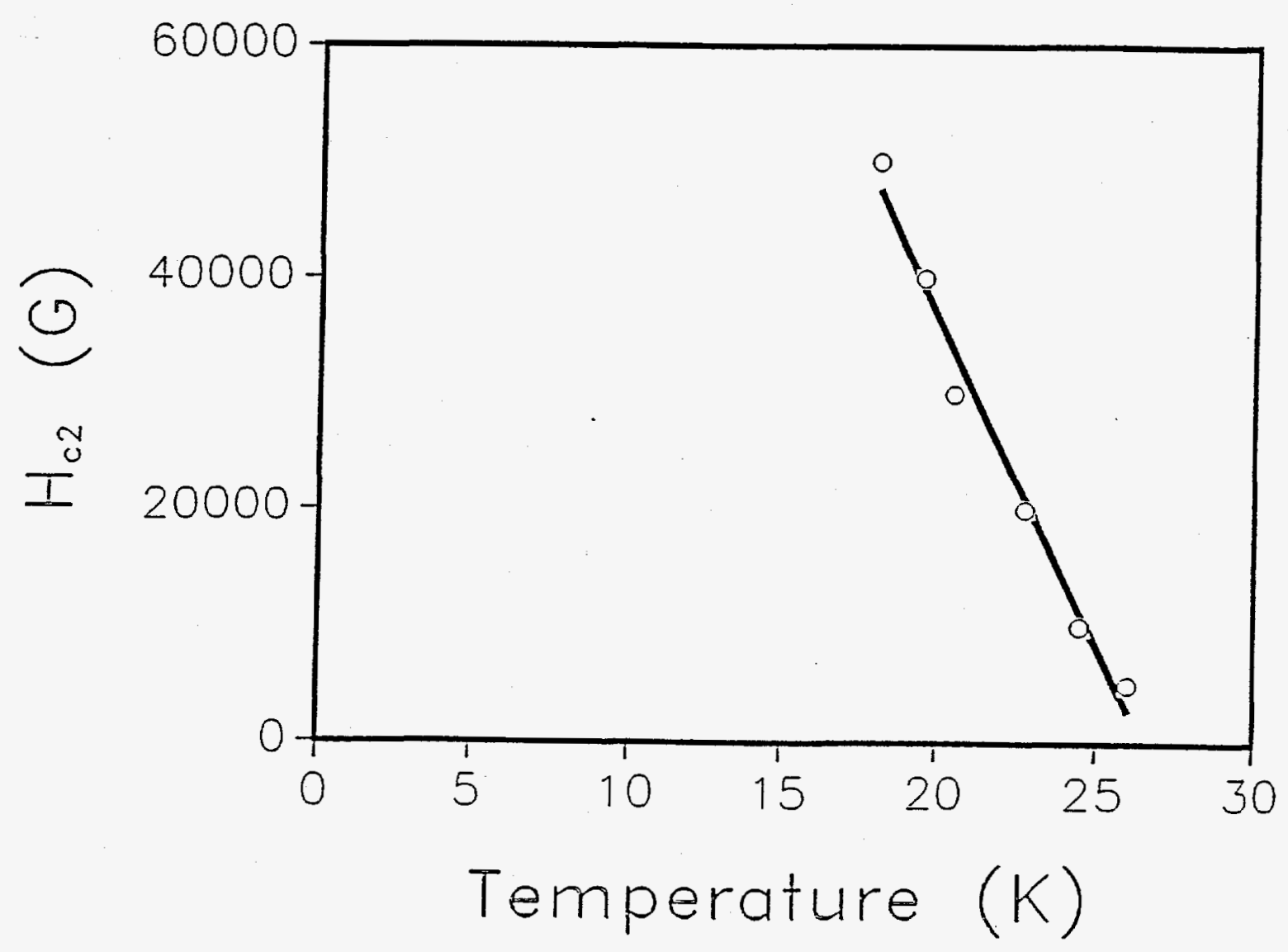

Figure 3.5: Upper critical field $H_{c 2}$ versus temperature 
Then the difference between $\mu \mathrm{SR}$ and magnetization penetration depths when the temperature is close to the critical temperature could be explained by the field dependence of the penetration depth. This tendency that the $\mu \mathrm{SR}$ penetration depth changes faster with temperature near $T_{\mathcal{C}}$ than the magnetization penetration depth can also be seen in other studies [28] [29] [96] [39] [43].

From the onset of superconductivity as seen in the $M$ versus $T$ data in Fig. 3.3, we get the critical field $H_{c 2}=-0.56($ Tesla/K) $T+14.9$ Tesla as shown in Fig. 3.5, from which we obtain $T_{c}(H=0)=26 \mathrm{~K}$. Thus,

$$
T_{c}(K)=26+\frac{1}{\frac{d H_{c 2}}{d T}} H(T)=26-\frac{H(T)}{0.56} .
$$

For the reason that our fitting must be in the reversible range, the fitting range in the $M(H)$ plots changes for different $T$ values. The middle of our fitting range can be approximately written as $H(T)=-0.19 T(K)+4.9$, which in turn gives the relation between the critical temperature and temperature to which the $M(H)$ curve was fitted

$$
T_{c}=17.3+0.34 T
$$

Therefore, $T_{c}$ is changing when the $T$ changes. Our fitting range is $14 \mathrm{~K}$ to $24 \mathrm{~K}$, which leads us to the average range $\bar{T}=19 \mathrm{~K}$ with an error bar $2 \mathrm{~K}$. From Fig. 3.4 we see that within the error of experiment, the $\mu \mathrm{SR}$ penetration depth matches the magnetization penetration depth data for $T \leq 15 \mathrm{~K}$.

In our sample we did not find any sign of a node in the gap (i.e., the penetration depth for $T \leq 10 \mathrm{~K}$ is alomost independent of $T)$. We also observed that our $\lambda(0)$ data satisfy Uemura's linear $T_{c}$ versus $\frac{1}{\lambda^{2}}$ relation [43].

The $\kappa$ value at low temperatures is almost constant, equal to 40 . Then, the 
coherence length for the system in the low temperature limit is $\xi=\lambda / \kappa=85 \AA$. 


\section{CHAPTER 4. MAGNETIC PROPERTIES OF $\mathrm{BaCuO}_{2+x}$}

\section{Introduction}

The structures of all known high transition temperature $T_{c}$ cuprate superconductors contain $\mathrm{CuO}_{2}$ planes [44]. The parent (undoped) insulator (at $T=0$ ) phases of those materials, like $\mathrm{La}_{2} \mathrm{CuO}_{4}$ and $\mathrm{YBa}_{2} \mathrm{Cu}_{3} \mathrm{O}_{6}$, exhibit long-range antiferromagnetic (AF) ordering of the $\mathrm{Cu}^{+2}\left(\mathrm{~d}^{9}\right)$ magnetic moments below a Néel temperature $T_{N}<550 \mathrm{~K}[45][11]$, and dynamic two-dimensional AF short-range ordering above $T_{N}[46]$ [101]. Furthermore, upon doping with either additional oxygen or by cation substitutions, the insulating parent materials become metallic and $T_{N}$ drops to zero, but short range AF order survives [11]-[48]. The short range AF order may play an important role in the mechanism of the high temperature superconductivity [49]. The strong AF interaction $(J \sim 1500 \mathrm{~K})$ between the $\mathrm{Cu}$ spins in the $\mathrm{CuO}_{2}$ planes [11] [101] originates from the $180^{\circ}$-bond-angle $\mathrm{Cu}^{+2}-\mathrm{O}^{-2}-\mathrm{Cu}^{+2}$ indirect superexchange interaction [50] [51]. Aharony et al. [52] and others (e.g. [53]) have argued that an intervening $\mathrm{O}^{-1}$ ion produced by a localized doped hole on the $\mathrm{O}^{-2}$ ion results instead in an indirect ferromagnetic interaction between the two adjacent $\mathrm{Cu}$ spins. This ferromagnetic interaction would result in magnetic frustration which may be relevant to the superconducting pairing mechanism in the high $T_{c}$ cuprates [52]. However, an alternative cause of FM interactions has been predicted to be a 
change in the $\mathrm{Cu}^{+2}-\mathrm{O}^{-2}-\mathrm{Cu}^{+2}$ bond angle from $180^{\circ}$ to $90^{\circ}$ [51]. This bond angle has recently been found by Büchner et al. [54] to be important to the occurrence of superconductivity. Their experiments show that when the tilt angle of the $\mathrm{CuO}_{6}$ octahedra in $\mathrm{La}_{2-x-y} \mathrm{Sr}_{x} \mathrm{Nd}_{y} \mathrm{CuO}_{4}$ decreased below $3.6^{\circ}$, the superconductivity was destroyed. Thus it is important to further clarify the conditions under which FM versus $\mathrm{AF} \mathrm{Cu}{ }^{+2}-\mathrm{Cu}^{+2}$ interactions occur in copper oxides.

A FM Cu ${ }^{+2}-\mathrm{Cu}^{+2}$ interaction has been found in several copper oxide compounds. For example, in $\mathrm{La}_{4} \mathrm{Ba}_{2} \mathrm{Cu}_{2} \mathrm{O}_{10}$ a bulk three dimensional ferromagnetic transition at $5.2 \mathrm{~K}$ was detected [55]. The structure of this compound contains isolated square-planar $\mathrm{CuO}_{4}$ units. The planes of adjacent $\mathrm{CuO}_{4}$ units are nearly perpendicular to each other. The $\mathrm{Cu}-\mathrm{O}-\mathrm{Cu}$ angle is $114.2^{\circ}$, but the respective $\mathrm{Cu}-\mathrm{O}$ and $\mathrm{O}-\mathrm{Cu}$ distances are $1.82 \AA$ and $3.80 \AA$. Since the latter distance is much larger than the sum of the $\mathrm{Cu}^{+2}$ and $\mathrm{O}^{-2}$ ionic radii, the $\mathrm{FM} \mathrm{Cu}$-Cu coupling is very weak $(J=2.6 \mathrm{~K}[55])$. Another example is $\mathrm{Li}_{2} \mathrm{CuO}_{2}$ [56] [57], in which square-planar $\mathrm{CuO}_{4}$ units are edge-shared to form infinite chains. The intrachain $\mathrm{Cu}^{+2}-\mathrm{O}^{-2}-\mathrm{Cu}^{+2}$ bond angle is $94.0^{\circ}$, and the $\mathrm{Cu}^{+2}-\mathrm{O}^{-2}$ distance is $1.96 \stackrel{\AA}{A}$ [56]. Specific heat and magnetic susceptibility measurements show an AF ordering transition at $T_{N}=9.3 \mathrm{~K}$ [58]. However, neutron diffraction measurements revealed that below $T_{N}$, the spins in the chains are ferromagnetically aligned as expected from the $\mathrm{Cu}^{+2}-\mathrm{O}^{-2}-\mathrm{Cu}^{+2}$ bond angle, and the AF character below $T_{N}$ arises from AF alignment of the spins in adjacent chains [56] [59].

The material studied in this work is $\mathrm{BaCuO}_{2+x}$. This compound has a large body-centered-cubic unit cell $\left(a_{o}=18.277 \AA\right)$ with 90 formula units/cell [60] [61]. As illustrated in Fig. 4.1, the cell contains 6 lone $\mathrm{CuO}_{2}$ units, $8 \mathrm{Cu}_{6} \mathrm{O}_{12}$ ring clusters 
[Fig. 4.1(b)] and $2 \mathrm{Cu}_{18} \mathrm{O}_{36}$ sphere clusters [Fig. 4.1(c)]. Within the ring clusters, the $\mathrm{Cu}^{+2}-\mathrm{O}^{-2}-\mathrm{Cu}^{+2}$ bond angle is $82.83^{\circ}$, the $\mathrm{Cu}^{+2}-\mathrm{O}^{-2}$ distance is $1.969 \stackrel{\circ}{A}$, and the $\mathrm{Cu}^{+2}-\mathrm{Cu}^{+2}$ distance is $2.605 \AA$, whereas within the sphere clusters, the $\mathrm{Cu}^{+2}-\mathrm{O}^{-2}-\mathrm{Cu}^{+2}$ bond angle is $86.86^{\circ}$, the $\mathrm{Cu}^{+2}-\mathrm{O}^{-2}$ distance is $1.98 \stackrel{\AA}{A}$, and the $\mathrm{Cu}^{+2}-\mathrm{Cu}^{+2}$ distance is $2.698 \AA$ [61]. A picture of the unfolded ring is shown in Fig. 4.1(d). Because these bond angles are rather close to $90^{\circ}$, one would expect the $\mathrm{Cu}$ spins in the $\mathrm{Cu}_{6}$ and $\mathrm{Cu}_{18}$ clusters to be ferromagnetically coupled.

By considering the atomic position occupancies of $\mathrm{BaCuO}_{2+x}$ from the structure determined using single crystal x-ray diffraction, Kipka and Müller-Buschbaum [60] obtained the composition $\mathrm{BaCuO}_{2}$. Similarly, Weller and Lines [61] found the compositions of two samples to be $\mathrm{BaCuO}_{2}$ and $\mathrm{BaCuO}_{2.07}$. They also measured the oxygen content of a sample by means of thermogravimetric analysis (TGA). They heated their sample to $800{ }^{\circ} \mathrm{C}$ under $\mathrm{N}_{2}+5 \% \mathrm{H}_{2}$ at $30{ }^{\circ} \mathrm{C} / \mathrm{min}$. The weight loss gave the composition of the initial material as $\mathrm{BaCuO}_{2.08} \pm 0.02$. From structural analysis on a single crystal, Paulus et al. [62] found the oxygen content to be 2.28 by modifying the crystal model obtained by Kipka et al. [60]. Aranda et al. [63] used both $\mathrm{x}$-ray and neutron diffraction methods, and obtained the composition $\mathrm{Ba}_{44} \mathrm{Cu}_{48}\left(\mathrm{CO}_{3}\right)_{6} \mathrm{O}_{81+x}$, (with $x=6.9(3)$ for their sample) instead of $\mathrm{BaCuO}_{2+x}$. Using iodometric titration, Eriksson et al. [64] found the oxygen content to be between 1.8 and 2.05. Therefore the oxygen content of $\mathrm{BaCuO}_{2+x}$ appears to be variable [65].

Specific heat measurements have been reported by Eckert et al. [67]. The results vary from sample to sample below $\sim 15 \mathrm{~K}$. For a sample prepared in air, a (magnetic) phase transition was found at about $13 \mathrm{~K}$. 

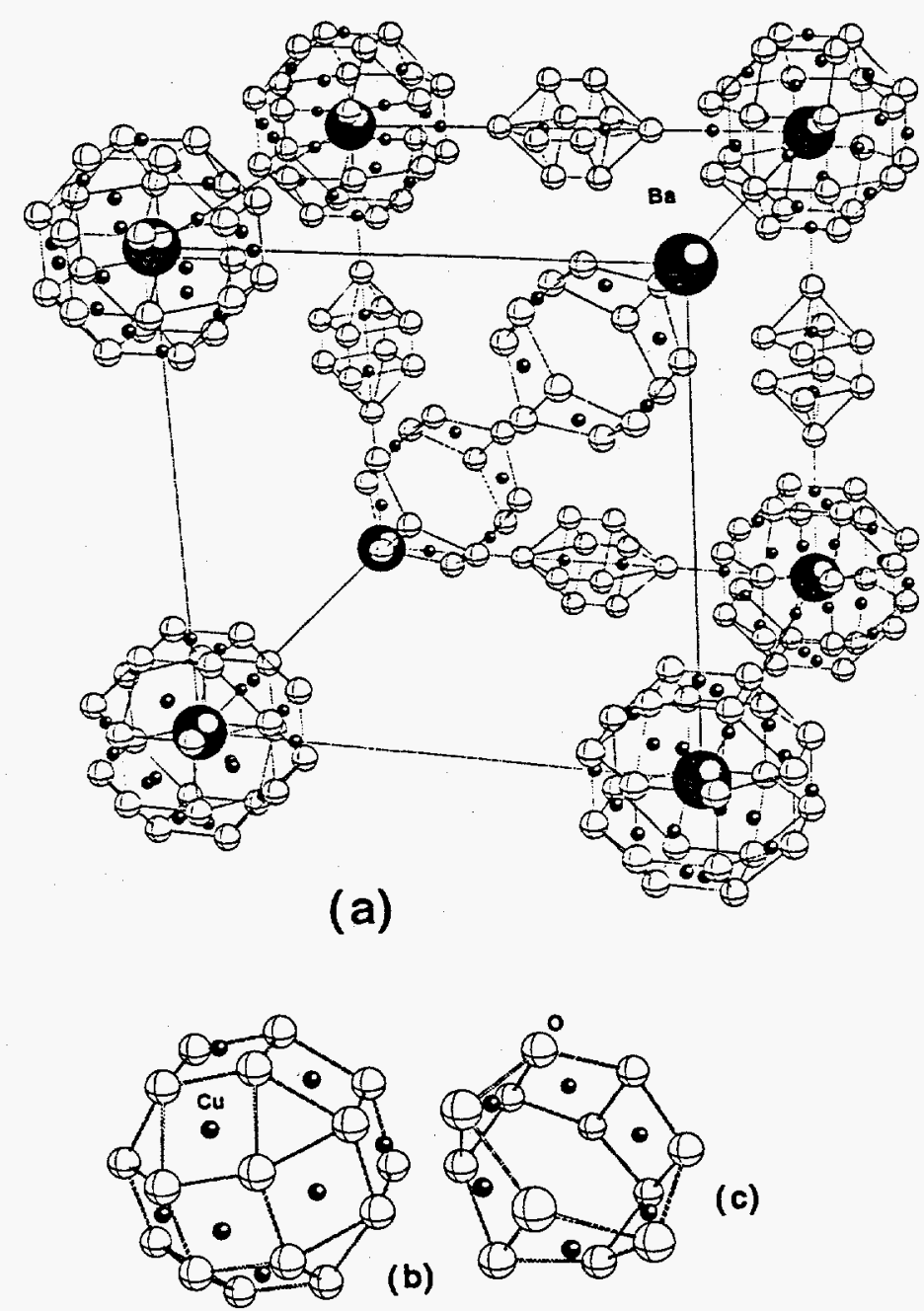

(c)

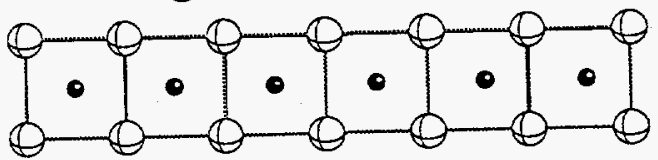

(d)

Figure 4.1: Perspective representation of the two types of $\mathrm{Cu}-\mathrm{O}$ clusters in the bcc unit cell of $\mathrm{BaCuO}_{2+x}$. The sphere-like clusters are located at the $(000)$ and $(1 / 21 / 21 / 2)$ (not shown) the ring-like clusters are located at the $(1 / 41 / 41 / 4)$ and the remaining seven equivalent positions with their axis of highest symmetry along the corresponding body diagonal (only two rings are shown). The lone spins are located along principal directions adjacent to the spheres (partially occupied). Both clusters consist of closed one-dimensional strips of $\mathrm{Cu}_{4} \mathrm{O}$ oxygen edge-sharing squares. 
Electrical conductivity $\sigma$ measurements have been reported by Migeon et al. [68]. The conductivity was found to be activated, with an activation energy of $0.61 \mathrm{eV}$ for $\mathrm{BaCuO}_{2}$, but which decreased with increasing oxygen content. A slope change was found in $\log (\sigma)$ versus $1 / T$ at $\approx 370 \mathrm{~K}[69]$, for both $\mathrm{BaCuO}_{2}$ (cubic) and $\mathrm{BaCuO}_{2.5}$ (orthorhombic) samples.

The magnetic properties of $\mathrm{BaCuO}_{2+x}$ have been previously measured by many research groups. From electron spin resonance (ESR) measurements, Vier et al. [70] and de Mesquita et al. [71] concluded that the resonance observed at room temperature originated from $\mathrm{Cu}^{2}+\left(3 \mathrm{~d}^{9}\right)$. Vier et al. [70] found the peak-to-peak linewidth to have a minimum at about $15 \mathrm{~K}$ which can be associated with a magnetic phase transition, but the magnetic structure was unknown. They also measured the DC magnetic susceptibility and found that the susceptibility $\chi$ versus temperature $\mathrm{T}$ curve can be characterized into two parts according to temperature range. For $T \geq$ $100 \mathrm{~K}$, the $1 / \chi$ versus temperature plot shows a linear behavior which corresponds to an effective magnetic moment $\mu_{e f f}$ of $1.72 \mu_{B}$ per $\mathrm{Cu}$ ion; at lower temperatures, the $1 / \chi$ versus $T$ curve shows positive curvature and $\mu_{e f f}$ increases, reaching a value of $3.16 \mu_{B}$ below $30 \mathrm{~K}$. Recently Petricek et al. [72] reported the observation of an antiferromagnetic to diamagnetic transition at about $3 \mathrm{~K}$ from $\chi(\mathrm{T})$ measurements for their sample of $\mathrm{BaCuO}_{2.5}$ (with a non-cubic structure).

Herein, we report ac and dc magnetization measurements of $\mathrm{BaCuO}_{2}$ from $2 \mathrm{~K}$ to $400 \mathrm{~K}$ in magnetic fields up to $55 \mathrm{kG}$. Our study was initially motivated by the observation of an anomalously strong, crystallographically forbidden (111) peak in the neutron diffraction pattern of this compound at $4 \mathrm{~K}$ which was not present at $300 \mathrm{~K}$, suggesting that this reflection was magnetic in origin [95]. On the other 
hand, the intensity of this peak was comparable to those of adjacent structural peaks, and the various magnetic structures modeled could not explain the intensity of this peak and the simultaneous absence of other magnetic peaks. We successfully resolved the nature and provided a model for the magnetic structure of the magnetic phase below $15 \mathrm{~K}$ via analysis of the present measurements. This model has been verified from analyses of subsequent polarized and unpolarized magnetic neutron diffraction measurements [74] [66]. Following the experimental details in the next section, we present our magnetization data, analyze them and present our model of the magnetic structure below $T_{N}$. We conclude with a discussion of the results. Some of our results were briefly presented previously [74].

\section{Experimental Details}

\section{Sample Preparation and Oxygen Content}

To synthesize $\mathrm{BaCuO}_{2+x}$, a one-to-one molar ratio mixture of $\mathrm{BaCO}_{3}(99.99$ \%) and $\mathrm{CuO}(99.99 \%)$ was ground thoroughly and heated in air to $800^{\circ} \mathrm{C}$ for 24 hours. The sample was then reground and fired five times in air at $925^{\circ} \mathrm{C}$ for 24 hours. The sample weight was recorded before and after each heat treatment. The final total weight loss of the batch was $13.663 \%$, giving a final product composition of $\mathrm{BaCuO}_{2.39}$, assuming that all the $\mathrm{CO}_{2}$ gas in the $\mathrm{BaCO}_{3}$ was released. The oxygen content of the batch was also measured using TGA by heating to $950^{\circ} \mathrm{C}$ in $5 \% \mathrm{H}_{2} / \mathrm{He}$ at a rate of $2{ }^{\circ} \mathrm{C} / \mathrm{min}$ and holding at $950^{\circ}$ for $180 \mathrm{~min}$. The weight did not change during the last $100 \mathrm{~min}$ of this $180 \mathrm{~min}$ period. The oxygen content from this measurement was found to be $2.56 \pm 0.03$. Portions of the batch were annealed in $\mathrm{He}$ or $\mathrm{O}_{2}$ at $900^{\circ} \mathrm{C}$ for 10 hours and cooled to room temperature at a rate of $10^{\circ} \mathrm{C} / \mathrm{hr}$. 
The oxygen content for the $\mathrm{O}_{2}$-annealed sample was then measured using TGA by heating the sample in $5 \% \mathrm{H}_{2} / \mathrm{He}$ to $950^{\circ} \mathrm{C}$ at a rate of $0.5^{\circ} \mathrm{C} / \mathrm{min}$, as shown in Fig. 4.2 (top), where the weight does not change above $820^{\circ} \mathrm{C}$. From the weight loss, the oxygen content of the $\mathrm{O}_{2}$-annealed sample was found to be $2.6 \pm 0.1$. The oxygen content for the He-annealed sample was measured using TGA in $5 \% \mathrm{H}_{2} / \mathrm{He}$ at a rate of $5.0^{\circ} \mathrm{C} / \mathrm{min}$ and kept at $950^{\circ} \mathrm{C}$ for $30 \mathrm{~min}$, as shown in Fig. 4.2 (bottom). From the weight loss, we calculated the oxygen content for the He-annealed sample to be $2.1 \pm 0.1$.

\section{X-ray Analysis}

Powder X-ray diffraction measurements were carried out using a Rigaku Geigerflex diffractometer, with a curved graphite crystal monochromator, using $\mathrm{Cu} \mathrm{K}_{\alpha}$ radiation $(\lambda=1.5418 \AA)$. The $\mathrm{X}$-ray patterns for the He-annealed sample and $\mathrm{O}_{2}$ annealed sample are shown in Fig. 4.3(top) and Fig. 4.3(bottom) respectively. The peaks were indexed according to the structural model obtained by Kipka et al. [60] and Weller et al. [61], and no impurity peaks were found, fraction of impurity phase in our samples is therefore estimated to be less than $5 \%$. The peak positions were corrected for zero point shift and nonlinearity using $\mathrm{Si}$ as an internal standard. Lattice parameters were obtained using the least squares fitting program FINAX. The lattice parameter was found to be $\mathrm{a}_{o}=18.29 \pm 0.03 \AA$ for the He-annealed sample, and $\mathrm{a}_{o}=18.34 \pm 0.03 \AA$ for the oxygen-annealed sample. The lattice parameter thus appears to increase as the oxygen content increases, as also observed by Weller (18.277 - 18.286 $\AA$ ) [61], Migeon (18.28 - 18.31 $\AA$ ) [68], and Eriksson (18.27 - 18.32 A) [64] [65]. Our oxygen- and helium-annealed samples were found [66] from neutron 

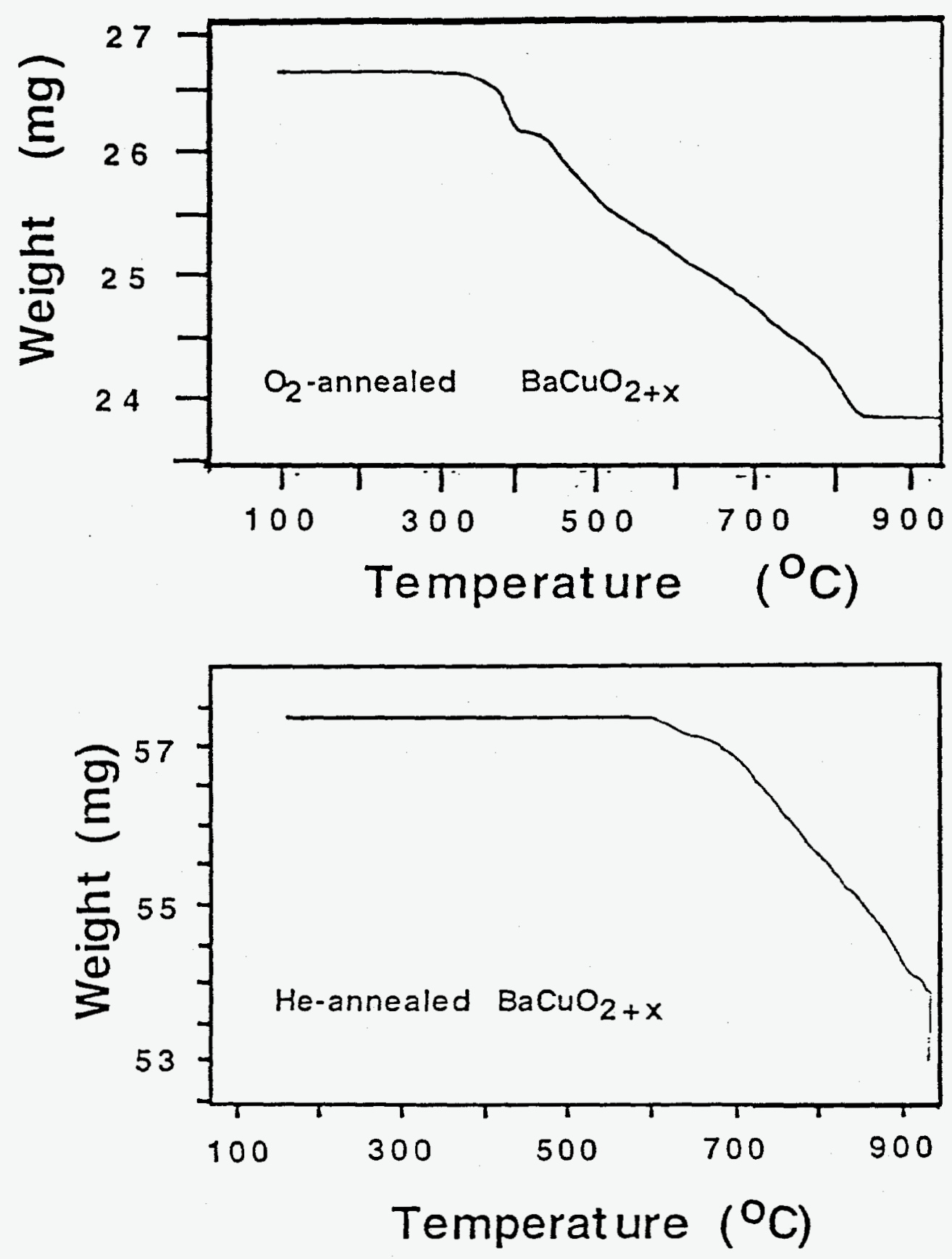

Figure 4.2: Thermogravimeteric analysis (TGA) data for $\mathrm{BaCuO}_{2+x}$ 
diffraction measurements to have essentially the same structure as proposed in [60] and [61].

\section{DC and AC Magnetization Measurements}

AC magnetic susceptibility measurements were made on a Lake Shore ACS7000 AC susceptometer. The amplitude of the AC field was kept at 100 Gauss and the frequency at $125 \mathrm{~Hz}$. The measurements versus temperature $T$ were performed under constant DC magnetic field in the range of 0 to $50 \mathrm{kG}$. DC magnetization $M$ data were obtained using a Quantum Design SQUID magnetometer. The $M(T)$ was measured at fixed applied magnetic fields of $H=500 \mathrm{G}$ and $H=10 \mathrm{kG}$, from $2 \mathrm{~K}$ to $400 \mathrm{~K}$, and $M(H)$ isotherms were measured at a series of different temperatures.

\section{Experimental Results and Preliminary Analysis}

The observed magnetic susceptibility $\chi$ of $\mathrm{BaCuO}_{2+x}$ is expected to be the sum of three terms: $\chi=\chi^{d i a}+\chi^{V V}+\chi^{\text {spin }}$, where the first term is due to the orbital diamagnetism of the closed-electron-shells of the atoms, the second is the orbital paramagnetic Van Vleck susceptibility of the $\mathrm{Cu}^{+2}$ ions and the third term is the spin susceptibility of the $\mathrm{Cu}^{+2}$ ions. From standard tables [75], one obtains $\chi^{d i a}=$ $-6.8 \times 10^{-5} \mathrm{~cm}^{3} / \mathrm{mol}$ for $\mathrm{BaCuO}_{2}$ and $\chi^{d i a}=-7.4 \times 10^{-5} \mathrm{~cm}^{3} / \mathrm{mol}$ for $\mathrm{BaCuO}_{2.5}$. The anisotropic $\chi^{V V}$ values for $\mathrm{Cu}^{+2}$ in the layered high $T_{c}$ cuprates have been estimated in, for example, Ref. [76]. For our samples, we take the powder average of those values and estimate that $\chi^{V V} \approx 7.4 \times 10^{-5} \mathrm{~cm}^{3} / \mathrm{mol}$. Thus, the sum $\chi^{d i a}+$ $\chi^{V V}$ is nearly zero and we will neglect it. Also, since $\chi \simeq \chi^{s p i n}$, we will henceforth denote $\chi^{\text {spin }}$ by $\chi$ to simplify notation. 


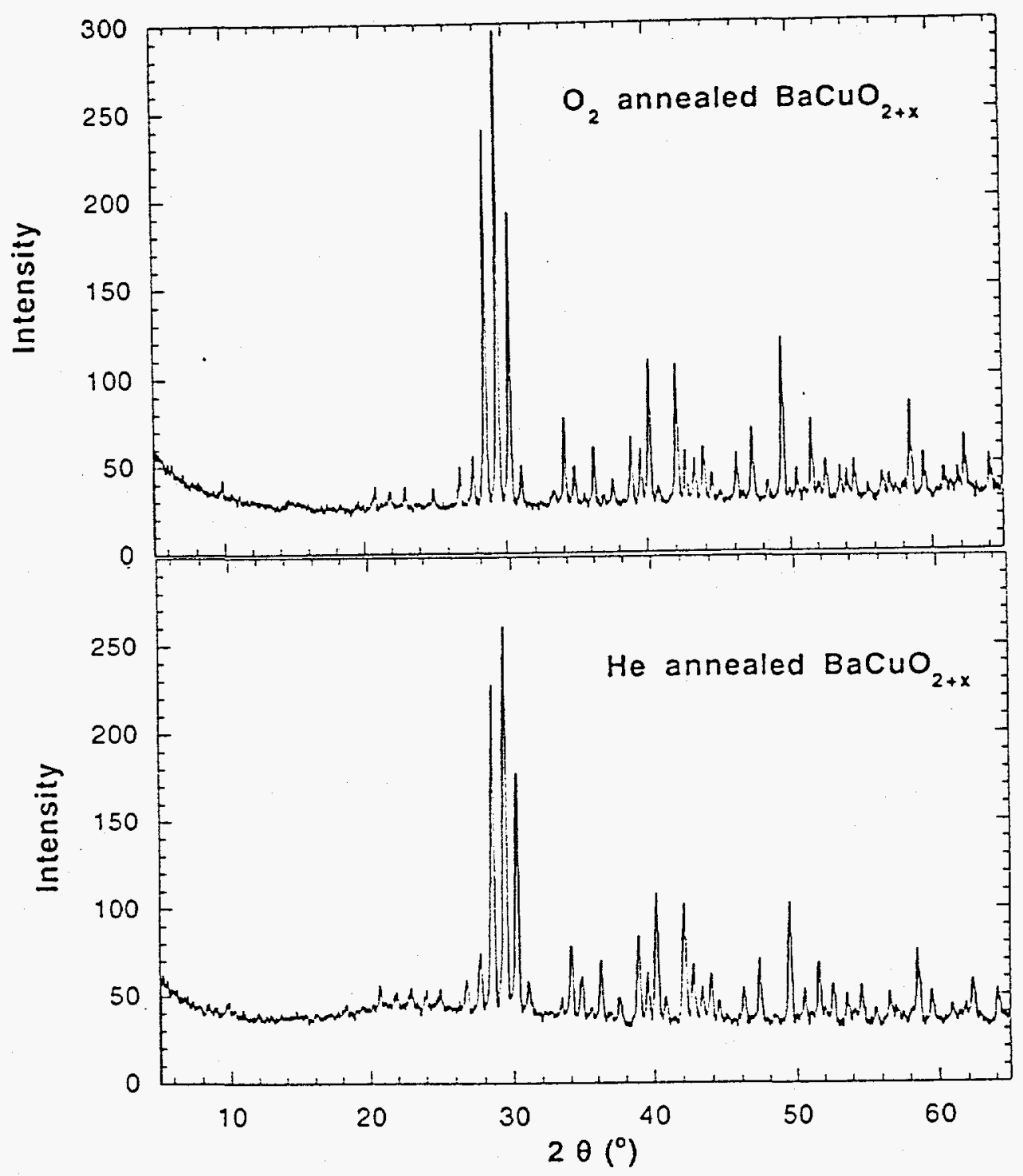

Figure 4.3: Powder $\mathrm{x}$-ray diffraction patterns for $\mathrm{BaCuO}_{2+x}$. 


\section{He-annealed $\mathrm{BaCuO}_{2+x}$}

We first present the results for the He-annealed sample of $\mathrm{BaCuO}_{2+x}$. Shown in Fig. 4.4(a) is the inverse molar magnetic susceptibility $\chi^{-1}$ versus $T$ of this sample. At high $T(\geq 300 \mathrm{~K}), \chi^{-1}(T)$ increases linearly with temperature (Curie-Weiss law):

$$
\chi^{-1}=(T-\theta) / C
$$

where

$$
C=\mathrm{N}_{\mathrm{A}} g^{2} S(S+1) \mu_{B}^{2} / 3 \mathrm{k}_{\mathrm{B}}=\mathrm{N}_{\mathrm{A}} \mu_{e f f}^{2} / 3 \mathrm{k}_{\mathrm{B}}
$$

is the molar Curie constant, $\mathrm{N}_{\mathrm{A}}$ is Avogadro's number, $g$ and $S$ are respectively the gyromagnetic factor and the spin of the paramagnetic species, $\mu_{B}$ is the Bohr magneton and $k_{B}$ is Boltzmann's constant. By fitting Eq. (4.1) to the data in the temperature range between 300 and $400 \mathrm{~K}$, we get $C=0.39 \mathrm{~cm}^{3} \mathrm{~K} / \mathrm{mole} \mathrm{Cu}, g=$ $2.10 \pm 0.04$ (assuming $S=1 / 2$ for $\mathrm{Cu}^{+2}$ ), $\theta=81 \mathrm{~K}$, and $\mu_{e f f}=1.82 \mu_{B} / \mathrm{Cu}$ atom, as indicated by the solid line in Fig. 4.4(a). The $g$ value obtained here is the same, within the error of measurement, as the powder average $\left(g=\left[\left(g_{x}^{2}+g_{y}^{2}+\right.\right.\right.$ $\left.\left.\left.g_{z}^{2}\right) / 3\right]^{1 / 2}=2.15\right)$ of the values $\left(g_{x}=2.27, g_{y}=2.12, g_{z}=2.05\right)$ obtained from the ESR measurements [70]. Thus, at high $T$, He-annealed $\mathrm{BaCuO}_{2+x}$ behaves as if all $\mathrm{Cu}^{+2}$ spins are equivalent and interacting ferromagnetically.

However, in contrast to the prediction of molecular field theory, (long range) FM order is not observed at (or below) $T=\theta=81 \mathrm{~K}$. Rather, $\chi^{-1}$ exhibits positive curvature with decreasing $T$ and then exhibits linear Curie-Weiss behavior again between $2 \mathrm{~K}$ and $6 \mathrm{~K}$ [Fig. $4.4(\mathrm{~b})$ ], with $C=1.13 \mathrm{~cm}^{3} \mathrm{~K} / \mathrm{mol} \mathrm{Cu}, \theta=0.4 \mathrm{~K}$, and $\mu_{e f f}=3.01 \mu_{B}$. The FM sign of $\theta$ and the large increase of $C$ with decreasing $T$ suggests that the $\mathrm{Cu}_{18}$ and $\mathrm{Cu}_{6}$ clusters in $\mathrm{BaCuO}_{2+x}$ have maximal spin 9 and 

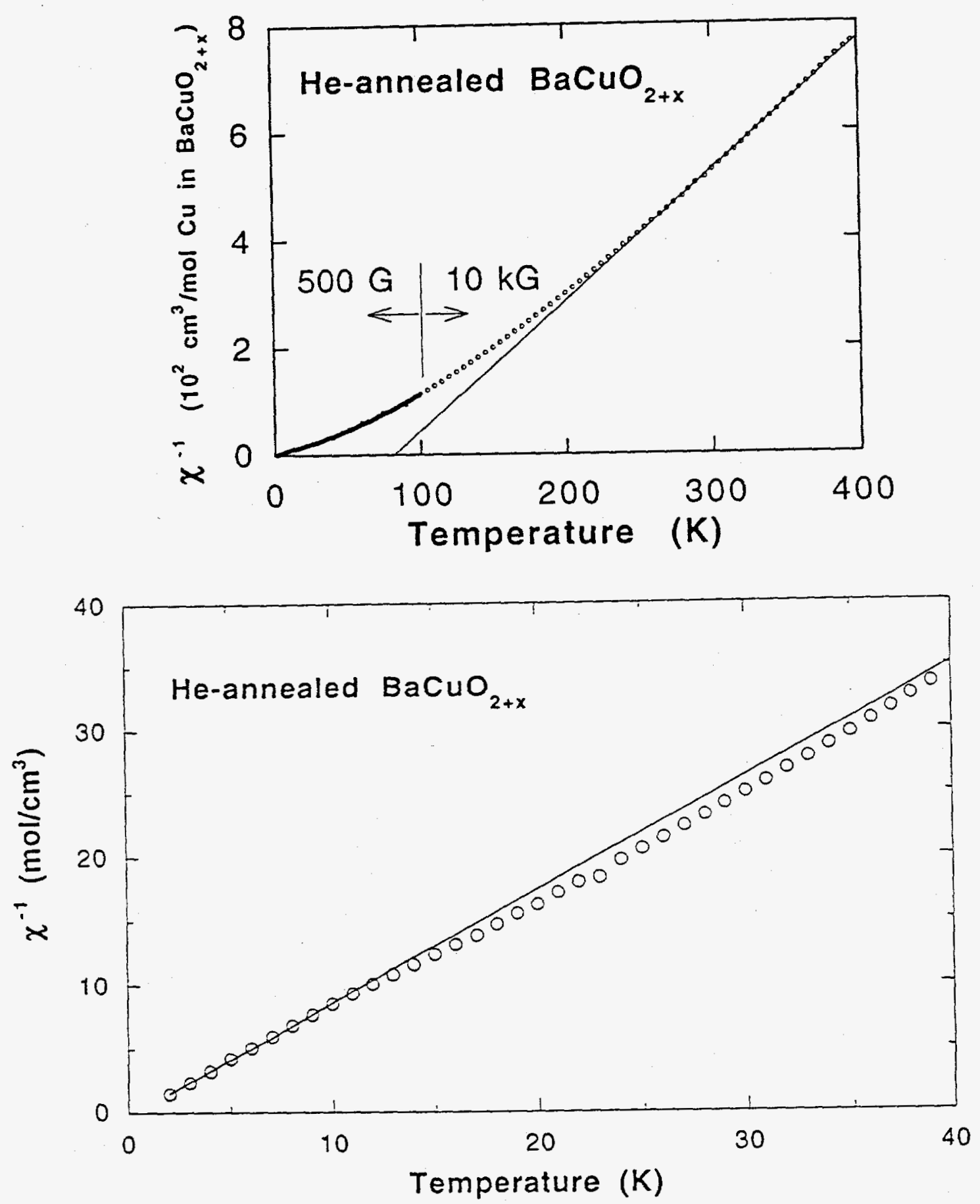

Figure 4.4: Inverse magnetic susceptibility $\chi^{-1}$ versus temperature for He-annealed $\mathrm{BaCuO}_{2+x}$ between $2 \mathrm{~K}$ to $400 \mathrm{~K}$. 
spin 3 ground states and spin $8,7, \ldots, 0$ and spin $2,1,0$ excited states, respectively. Indeed, the shape of $\chi^{-1}(T)$ in Fig. $4.4(\mathrm{a})$ is very similar to that of a $\left(\mathrm{Cr}^{+3}\right)_{4}$ cluster with a maximal spin 6 ground state and spin 5, 4, .., 0 excited states [77].

In order to further clarify the magnetic character of $\mathrm{BaCuO}_{2+x}, M(H)$ isotherms were obtained at various temperatures between $2 \mathrm{~K}$ and $300 \mathrm{~K}$, as shown in Fig. 4.5(a). At low temperature ( $T \leq 15 \mathrm{~K})$, the $M(H)$ data exhibit negative curvature. The slope of the data $\chi_{o}$ at the highest fields ( $30 \mathrm{kG}$ to $55 \mathrm{kG}$ ) exhibits a peak at a temperature between 15 to $25 \mathrm{~K}$, as shown in Fig. 4.5(b), suggesting the onset of long-range $\mathrm{AF}$ order below $T_{N} \sim 15$ to $25 \mathrm{~K}$. A higher resolution measurement of $M(T)$ at fixed $H=55 \mathrm{kG}$ is shown in Fig. 4.6. The data show a clear slope change at $T_{N} \approx 12 \pm 2 \mathrm{~K}$.

The AC susceptibility for the He-annealed sample was measured at various DC magnetic fields $H$, as shown in Fig. 4.7. The antiferromagnetic transition postulated above becomes very clear for $H \geq 40 \mathrm{kG}$. The data in Figs. 4.5(b), 6, and 7 together suggest that some of the $\mathrm{Cu}$ spins in $\mathrm{BaCuO}_{2+x}$ order antiferromagnetically at $T_{N}$ $\simeq 15 \mathrm{~K}$, and the remainder remain paramagnetic down to $2 \mathrm{~K}$.

\section{Oxygen-annealed $\mathrm{BaCuO}_{2+x}$}

Now we present the $\mathrm{DC}$ magnetic results for the $\mathrm{O}_{2}$-annealed sample of $\mathrm{BaCuO}_{2+x}$. Shown in Fig. 4.8 is $\chi^{-1}$ versus $T$ for this sample. At high temperature ( $\geq 300 \mathrm{~K}$ ), $\chi^{-1}(T)$ follows the Curie-Weiss law, Eq. 4.1 , with $C=0.35 \mathrm{~cm}^{3} \mathrm{~K} / \mathrm{mol} \mathrm{Cu}, g=$ 1.93, $\theta=65 \mathrm{~K}$, and $\mu_{\text {eff }}=1.67 \mu_{B} / \mathrm{Cu}$ atom, as shown by the fitted straight line in Fig. 4.8. By comparism with the above corresponding values for the He-annealed sample, one sees that the effective magnetic moment decreases as the oxygen content 

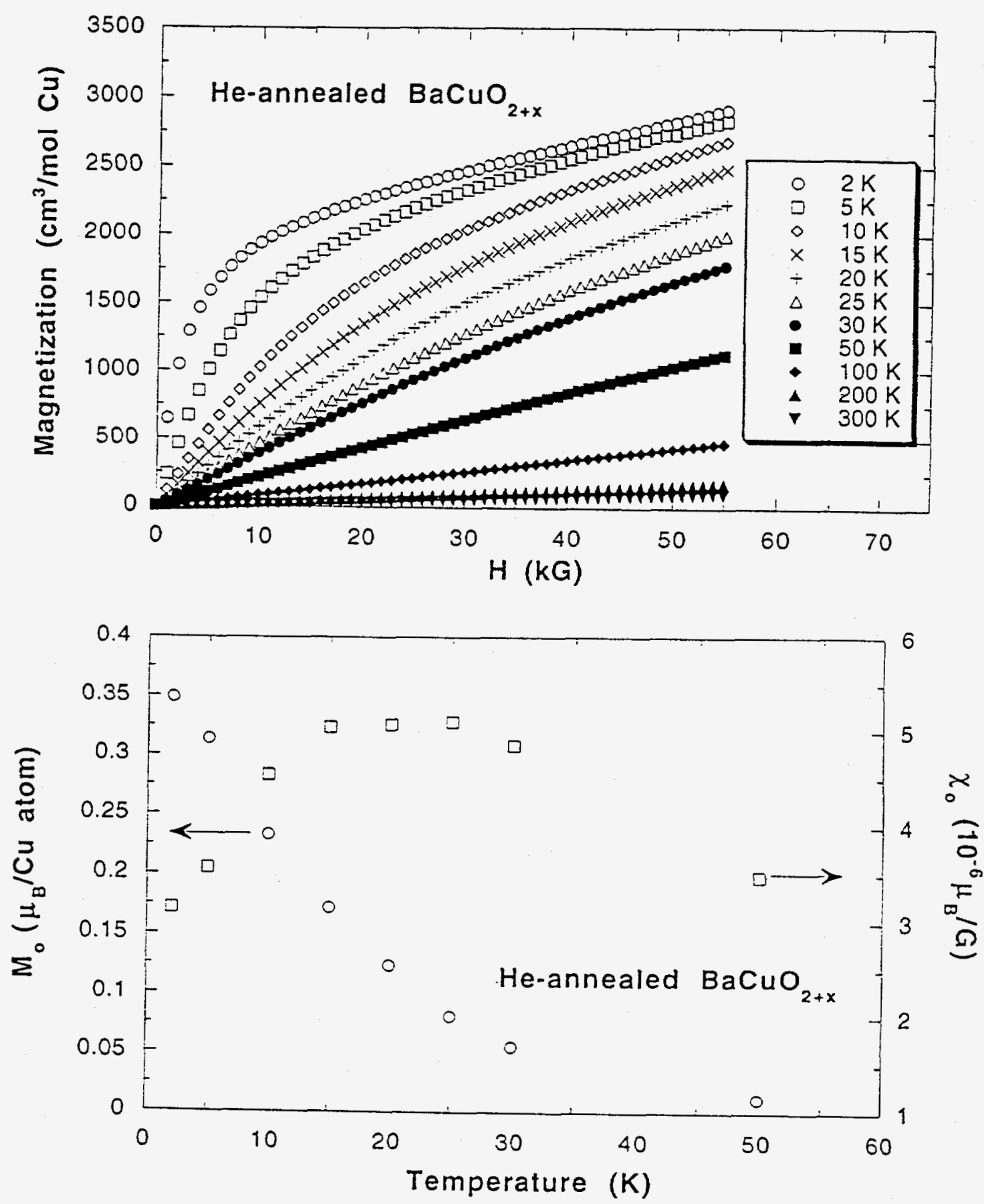

Figure 4.5: Top. Magnetization vs. applied magnetic field $H$ at various temperatures for $\mathrm{He}$-annealed $\mathrm{BaCuO}_{2+x}$. Bottom. Slope $\chi_{o}$ (right-hand-scale) and y-intercept $M_{O}$ (left-hand scale) of straight-line fits to the data in the top panel between $30 \mathrm{kG}$ and $55 \mathrm{kG}$. 


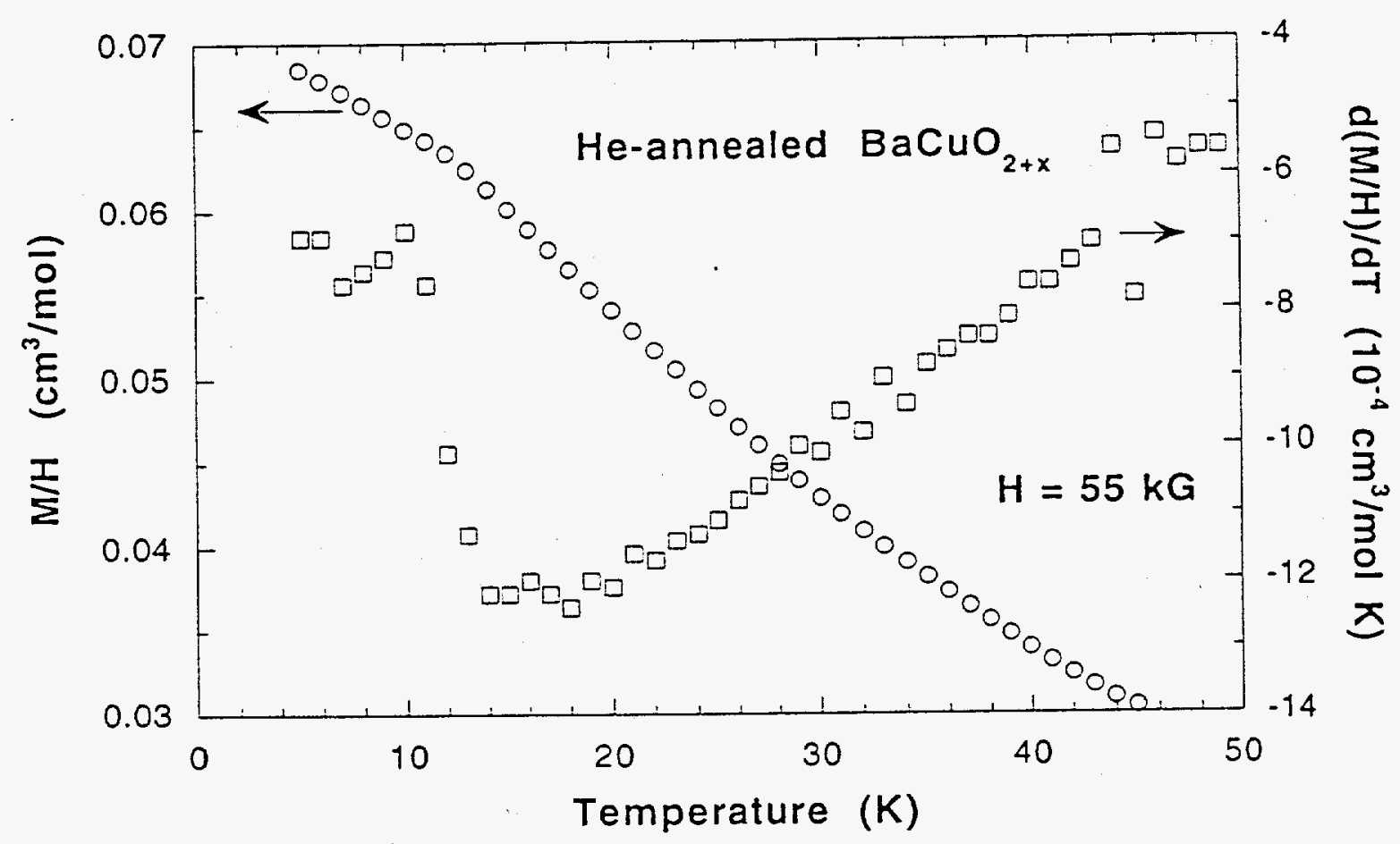

Figure 4.6: High field ( $H=55 \mathrm{kG}$ ) DC magnetization $M$ divided by $H$ versus temperature for $\mathrm{He}$-annealed $\mathrm{BaCuO}_{2+x}$ (open circles). The temperature derivative of these data versus temperature (open squares) is also shown. 


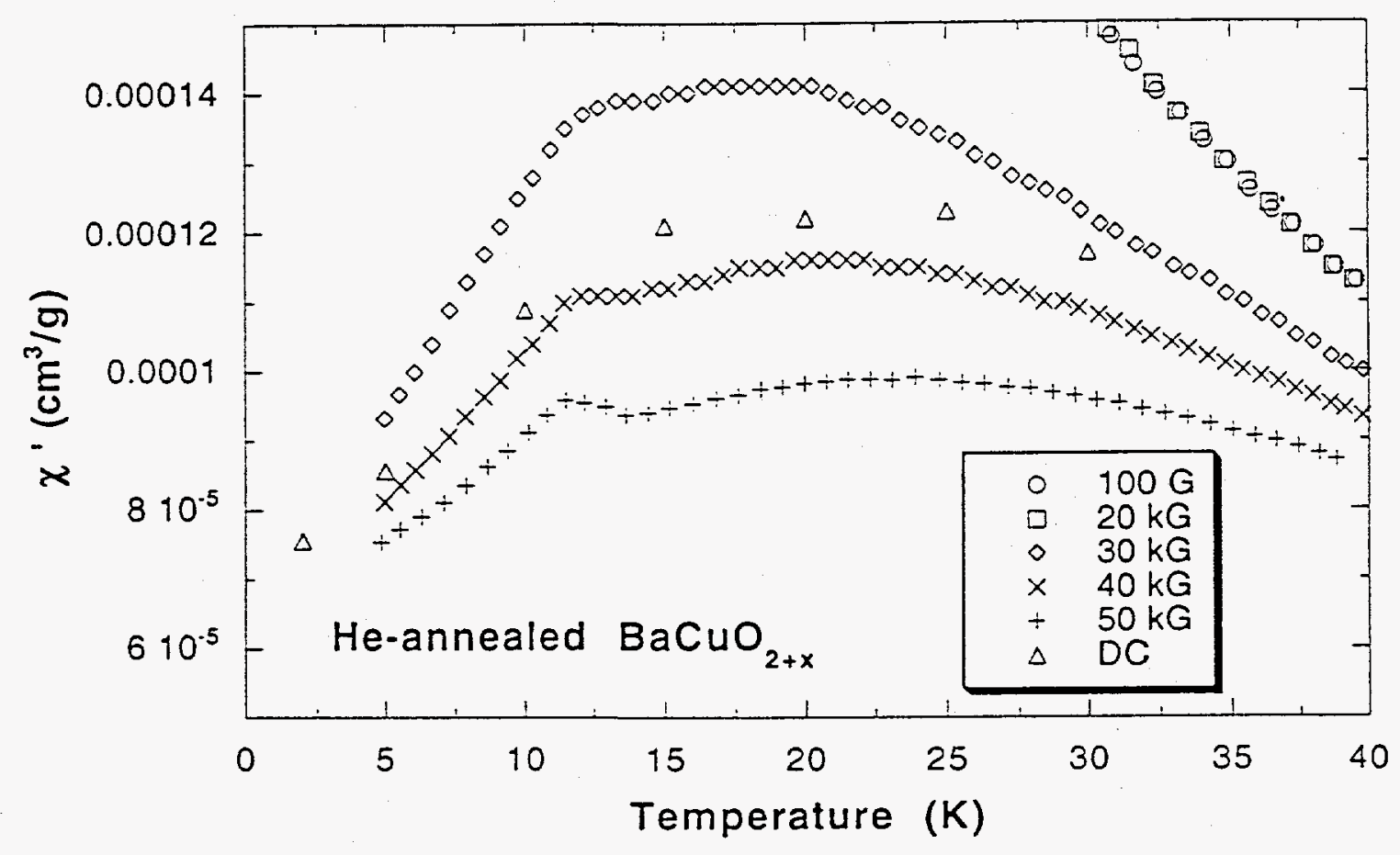

Figure 4.7: AC magnetic susceptibility $\chi^{\prime}$ in various DC fields versus temperature. The open triangles are the high-field differential susceptibility data $\chi_{o}(T)$ reproduced from Fig. 4.5(Bottom). 
increases, as first observed by Migeon [68].

The $M(H)$ isotherms measured at various temperatures between $2 \mathrm{~K}$ and $300 \mathrm{~K}$ are shown in Fig. 4.9(a). The high-field (30-50 kG) slope $\left(\chi_{o}\right)$ and corresponding y-intercept $\left(M_{O}\right)$ are plotted versus temperature in Fig. 4.9(b).

\section{Detailed Analysis and Modeling for Helium-annealed Sample}

\section{Magnetization versus Magnetic Field and Temperature for $T \ll T_{N}$}

From Fig. 4.5(a), the $M(H)$ data at the lowest temperatures contain a component which saturates by $H \sim 20 \mathrm{kG}$, and a component with magnetization proportional to $H$. In this high field linear region, we parametrize this behavior as follows:

$$
M(H)=M_{o}+\chi_{o} H
$$

By subtracting the fitted $\chi_{o} H$ from the observed $M(H)$, we can examine the behavior of the saturating contribution, normalized to the highest-field value, as shown in Fig. 4.10 for the data in Fig. $4.5(\mathrm{a})$ at $2 \mathrm{~K}$, where $M(2 \mathrm{~K})=31 \mu_{B} /$ unit cell. Also plotted are Brillouin functions $B_{S}(H, T)$ for $g=2, T=2 \mathrm{~K}$, and spins $S=6$, 9 and 15. A comparison of the shapes of these Brillouin functions with the data shows that the saturation arises from a large spin $S \sim 9$. The only candidate in the structure which could have this spin value is the $\mathrm{Cu}_{18}$ sphere cluster which we therefore conclude remains paramagnetic to $2 \mathrm{~K}$ with a ground state with maximal spin $S_{s}=18(1 / 2)=9$. The $\mathrm{Cu}_{6}$ rings thus apparently do not contribute to the saturation and their contributions to $M(H)$ must therefore be contained in the $\chi_{0} H$ term in Eq. (4.3). The Curie constant expected from Eq. (4.2) for the low-field 


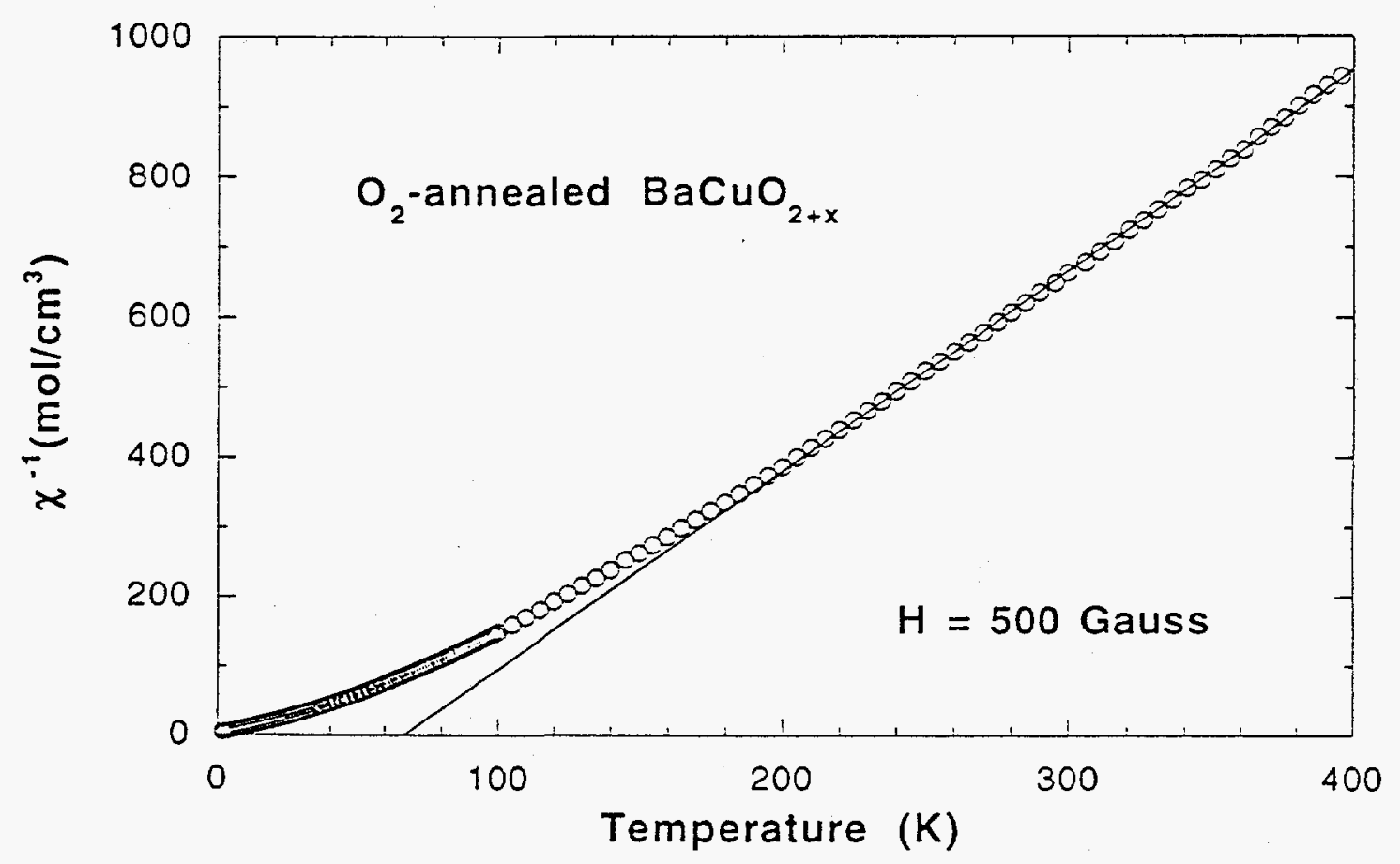

Figure 4.8: Inverse magnetic susceptibility $\chi^{-1}$ versus temperature for $\mathrm{O}_{2}$-annealed $\mathrm{BaCuO}_{2+x}$ between $2 \mathrm{~K}$ and $400 \mathrm{~K}$. The solid line is a fit of Eq. (4.1) to the data above $300 \mathrm{~K}$. 

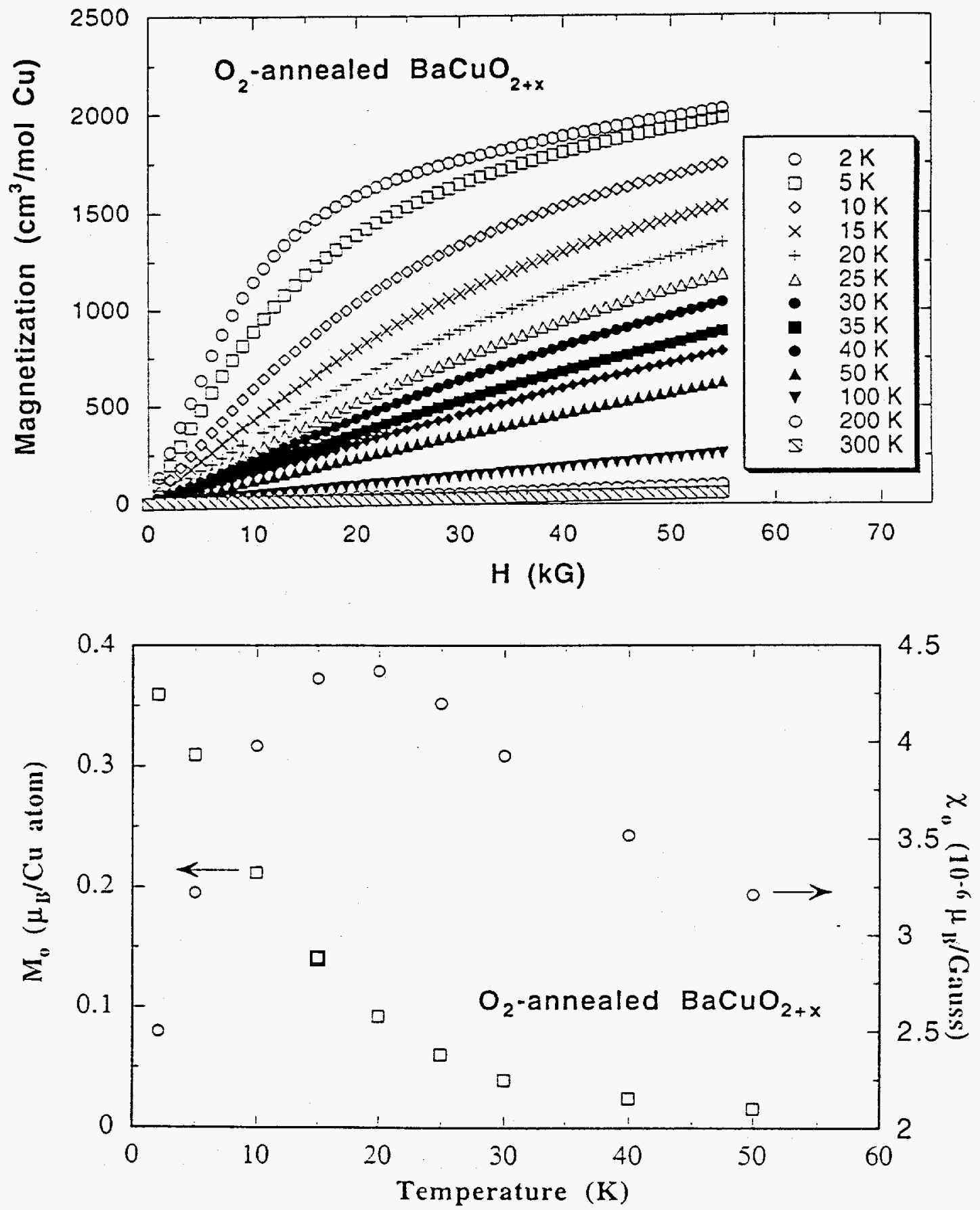

Figure 4.9: Top. Magnetization versus applied magnetic field $H$ at various temperatures for $\mathrm{O}_{2}$-annealed $\mathrm{BaCuO}_{2+x}$. Bottom. Slope $\chi_{0}$ (right-hand-scale) and y-intercept $M_{o}$ (left-hand-scale) of straight-line fits to the data in top panel between $30 \mathrm{kG}$ and $55 \mathrm{kG}$. 
Curie-Weiss-law susceptibility of the $\mathrm{Cu}_{18}$ clusters with $g=2.1$ (from above) is $C$ $=49.6 \mathrm{~cm}^{3} \mathrm{~K} / \mathrm{mol} \mathrm{Cu} 18$ clusters $=1.10 \mathrm{~cm}^{3} \mathrm{~K} /\left(\mathrm{mol} \mathrm{Cu}\right.$ in $\left.\mathrm{BaCuO}_{2+x}\right)$. This value is in close agreement with the value $\left(1.13 \mathrm{~cm}^{3} \mathrm{~K} / \mathrm{mol} \mathrm{Cu}\right.$ in $\left.\mathrm{BaCuO}_{2+x}\right)$ observed between $2 \mathrm{~K}$ and $6 \mathrm{~K}$ in Fig. 4.4(bottom), confirming that the $\mathrm{Cu}_{18}$ sphere clusters are primarily responsible for the Curie-Weiss susceptibility observed in Fig. 4.4(bottom) between $2 \mathrm{~K}$ and $6 \mathrm{~K}$, and that the $\mathrm{Cu}_{6}$ clusters do not contribute to the Curie-Weiss susceptibility in this $T$ range. The Weiss temperature $\theta=+0.4 \mathrm{~K}$ found from Fig. 4.4(bottom) suggests that the $\mathrm{Cu}_{18}$ sphere clusters should undergo long-range ferromagnetic intercluster order at roughly this temperature.

On the other hand, the above value of $M_{o}(2 K)=31 \mu_{B} /$ unit is about $6 \mu_{B}$ less than the value $M_{o}=2 g S_{s} \mu_{B} /$ unit cell $=37 \mu_{B} /$ unit cell expected for the two $\mathrm{Cu}_{18}$ clusters per unit cell, again using $g=2.10$. Since the six lone $\mathrm{Cu}$ ions in the unit cell would have a saturation moment of $6 g S_{C u} \mu_{B} /$ unit cell $\approx 6 \mu_{B} /$ unit cell, three lone $\mathrm{Cu}$ ions are apparently antiferromagnetically coupled to each $\mathrm{Cu}_{18}$ cluster in the unit cell (see Fig. 4.1), and their moments oppose that of the $\mathrm{Cu}_{18}$ cluster at low $T$ and $H$. Since the magnetic behaviors of the $\mathrm{Cu}_{18}$ clusters and the lone $\mathrm{Cu}$ ions are now accounted for, the AF ordering inferred to occur below $\sim 15 \mathrm{~K}$ from Fig. 4.5 (b) above must be due to the $\mathrm{Cu}_{6}$ ring clusters, which are the only remaining magnetic species in the unit cell.

To summarize the above discussion, our model thus far for the magnetic properties of $\mathrm{BaCuO}_{2+x}$ consists of the following. All of the $\mathrm{Cu}$ ions have an oxidation state of +2 and have spin $S_{C u}=1 / 2$. Each $\mathrm{Cu}_{18}$ sphere cluster has a maximal spin ground state with spin $S_{s}=9$, with lower spin excited states. This ground state is consistent with the ferromagnetic nature of the nearest-neighbor $\mathrm{Cu}-\mathrm{Cu}$ ex- 
change coupling within each cluster expected from the nearly $90^{\circ} \mathrm{Cu}-\mathrm{O}-\mathrm{Cu}$ bond angle, as noted in the Introduction. For the same reason, we also expect each $\mathrm{Cu}_{6}$ ring cluster to have a maximal spin ground state with spin $S_{r}=3$. From Fig. 4.4, both the $\mathrm{Cu}_{6}$ and $\mathrm{Cu}_{18}$ clusters are in their ground states below $\sim 40 \mathrm{~K}$. Below $T_{N}$ $\sim 15 \mathrm{~K}$, the magnetic moments of the $\mathrm{Cu}_{6}$ ring clusters with spins $S_{r}=3$ order antiferromagnetically with respect to each other. The magnetic moments of the lone copper ions with spins $S_{I}=1 / 2$ and of the $\mathrm{Cu}_{18}$ sphere clusters with spins $S_{s}=$ 9 do not participate in this AF ordering; these species remain paramagnetic down to our low temperature measurement limit of $2 \mathrm{~K}$. The spin of each $\mathrm{Cu}_{18}$ sphere cluster is antiferromagnetically coupled to those of three lone $\mathrm{Cu}$ ions; the spin of each lone $\mathrm{Cu}$ ion is antiferromagnetically coupled to that of one $\mathrm{Cu}_{18}$ sphere cluster. The gyromagnetic factor $\mathrm{g}$ of the $\mathrm{Cu}_{18}$ sphere clusters and the lone $\mathrm{Cu}$ ions are both close to 2 , and a similar value is expected for the $\mathrm{Cu}_{6}$ clusters. For $T \ll T_{N}$, the magnetization of the antiferromagnetically ordered $\mathrm{Cu}_{6}$ ring clusters is proportional to $H$, and we denote the magnetization of a single ring by $M_{r}=\chi_{r} H$.

We now proceed to calculate the magnetization versus magnetic field and temperature expected at $T \ll T_{N}$ for $\mathrm{BaCuO}_{2+x}$. On the basis of the above model, the measured magnetization $M(H, T)$ of a unit cell of $\mathrm{BaCuO}_{2+x}$ at $T \ll T_{N}$ is the sum of the respective contributions from the 6 lone $\mathrm{Cu}$ ions, $2 \mathrm{Cu}_{18}$ sphere clusters and $8 \mathrm{Cu}_{6}$ ring clusters:

$$
M(H, T)=6 M_{I}(H, T)+2 M_{s}(H, T)+8 \chi_{r}(T) H
$$

where $M_{I}(H, T)$ and $M_{s}(H, T)$ are respectively the magnetizations of a single lone $\mathrm{Cu}$ ion and $\mathrm{Cu}_{18}$ sphere cluster, respectively. The AF coupling between $M_{s}$ and $M_{I}$ is taken into account using molecular field theory. The effective magnetic fields seen 


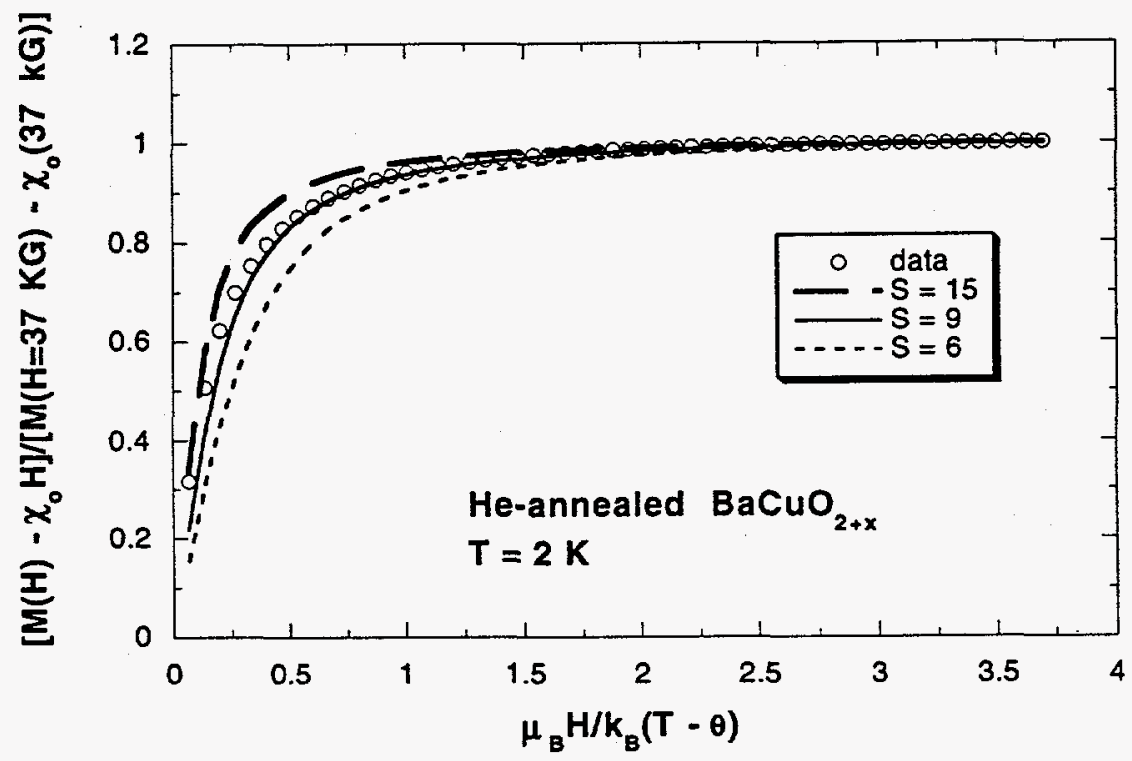

Figure 4.10: The saturating part $M(H)-\chi_{o} H$ of the magnetization $M(H)$ at $2 \mathrm{~K}$ for $\mathrm{He}$-annealed $\mathrm{BaCuO}_{2+x}$, from Fig. 4.5(Top), normalized to $M(H$ $=37 \mathrm{kG})-\chi_{0}(37 \mathrm{kG})$, open circles, plotted versus reduced magnetic field $\mu_{B} H / k_{B}(T-\theta)$, where $\theta=0.3 \mathrm{~K}$. Brillouin functions with $T$ replaced by $T-\theta, g=2$ and spins $S=6,9$ and 15 are also plotted versus reduced field. 
by $M_{I}(H, T)$ and $M_{s}(H, T)$ are then respectively

$$
\begin{aligned}
& H_{e f f, I}=H-\lambda M_{s} \\
& \text { and } \\
& H_{e f f, s}=H-3 \lambda M_{I},
\end{aligned}
$$

where $H$ is the applied magnetic field and $\lambda>0$ is the antiferromagnetic molecular field coupling constant between $M_{I}$ and $M_{s}$. In the molecular field approximation, these magnetizations are given by

$$
\begin{aligned}
& M_{I}(H, T)=g S_{I}{ }_{B} B_{S_{I}}\left(H_{e f f, I}, T\right) \\
& \quad \text { and } \\
& M_{s}(H, T)=g S_{s} \mu_{B} B_{S_{s}}\left(H_{e f f, s}, T\right),
\end{aligned}
$$

where the $g$ values of the $\mathrm{Cu}_{6}$ and $\mathrm{Cu}_{18}$ cluster ground state spins are assumed to be the same, and $B_{J}(H, T)$ is the Brillouin function for spin $J$.

Equations (4.5) and (4.6) constitute a set of coupled nonlinear equations. We solve them using an iterative procedure with ascending orders of approximation. To zeroth order,

$$
\begin{gathered}
H_{\text {eff,I }}^{(0)}=H_{\text {eff,s }}^{(0)}=H, \\
M_{I}^{(0)}(H, T)=g S_{I^{\mu}} B^{B} S_{I}\left(H_{e f f, I}^{(0)}, T\right), \\
\quad \text { and } \\
M_{s}^{(0)}(H, T)=g S_{s} \mu_{B} B_{S_{s}}\left(H_{e f f, s}^{(0)}, T\right) .
\end{gathered}
$$

To first order,

$$
H_{e f f, I}^{(1)}=\left[H-\lambda g S_{s} \mu_{B} B_{S_{s}}\left(H_{e f f, s}^{(0)}, T\right)\right],
$$


and

$$
\begin{gathered}
H_{e f f, s}^{(1)}=\left[H-3 \lambda g S_{I^{\mu}} B^{{ }^{B}} B_{S_{I}}\left(H_{e f f, I}^{(0)}, T\right)\right] \\
M_{I}^{(1)}(H, T)=g S_{I}^{\mu} B^{B} S_{I}\left(H_{e f f, I}^{(1)}, T\right) \\
\quad \text { and } \\
M_{s}^{(1)}(H, T)=g S_{s} \mu_{B} B_{S_{s}}\left(H_{e f f, s}^{(1)}, T\right)
\end{gathered}
$$

By induction to nth order,

$$
\begin{aligned}
& H_{e f f, I}^{(n)}=\left[H-\lambda g S_{s} \mu_{B} B_{S_{s}}\left(H_{e f f, s}^{(n-1)}, T\right)\right] \\
& \text { and } \\
& H_{e f f, s}^{(n)}=\left[H-3 \lambda g S_{I} \mu_{B} B_{S_{I}}\left(H_{e f f, I}^{(n-1)}, T\right)\right]
\end{aligned}
$$

and

$$
\begin{aligned}
& M_{I}^{(n)}(H, T)=g S_{I}^{\mu_{B}} B_{S_{I}}\left(H_{e f f, I}^{(n)}, T\right) \\
& \quad \text { and } \\
& M_{s}^{(n)}(H, T)=g S_{s} \mu_{B} B_{S_{s}}\left(H_{e f f, s}^{(n)}, T\right) .
\end{aligned}
$$

The zeroth order magnetizations correspond to no coupling between them. The influence of the coupling $(\lambda)$ is taken into account with increasing accuracy as the order of iteration increases. The accuracy of the calculated magnetization in second order is estimated to be $5 \%$ and in 20 th order to be $0.01 \%$. By solving Eqs. (4.12) numerically for $M_{I}(H)$ and $M_{s}(H)$ at $T=2 \mathrm{~K}$ using $g=2.10$ (above) for various $\lambda$ and $\chi_{r}(2 \mathrm{~K})$ and inserting these into Eq. (4.4), we obtained a reasonably good fit to the $M(H)$ data at $2 \mathrm{~K}$ in Fig. 4.9 (top) using $\lambda=0.22 \mathrm{~N}_{A} \frac{G^{2}}{e r g}(=390$ [dimensionless]) 
(2nd order iteration) and $\lambda=0.21 \mathrm{~N}_{A} \frac{\mathrm{G}^{2}}{\mathrm{erg}}$ (20th order iteration) and $\chi_{r}(2 \mathrm{~K})=8.1$ $\times 10^{-2} \mathrm{~cm}^{3} / \mathrm{mol} \mathrm{Cu}$ in $\mathrm{BaCuO}_{2+x}$ for both order approximations. The fit is shown as the solid curve in Fig. 4.11, where the separate contributions of the $\mathrm{Cu}_{6}$ clusters, the $\mathrm{Cu}_{18}$ clusters and the $\mathrm{Cu}$ ions are also shown.

The magnetizations at $2 \mathrm{~K}$ of a lone $\mathrm{Cu}$ ion $M_{I}$ and a $\mathrm{Cu}_{18}$ sphere cluster $M_{s}$ versus applied magnetic field $H$ in Fig. 4.11 can be understood as follows. The effective magnetic fields experienced by a $\mathrm{Cu}_{18}$ sphere cluster and a lone $\mathrm{Cu}$ ion are different. Below $H \sim 50 \mathrm{kG}$, the effective field $H_{e f f, s}=H-3 \lambda M_{I}$ seen by a sphere cluster is on the order of $H$ because the opposing exchange field due to AF coupling with the three lone $\mathrm{Cu}$ ions is relatively small compared with $H$. Therefore, the effective field seen by the $\mathrm{Cu}_{18}$ clusters is always in the direction of $H$. On the other hand, for $H$ below $\sim 20 \mathrm{kG}$, the effective field $H_{e f f, I}=H-\lambda M_{s}$ experienced by a lone $\mathrm{Cu}$ ion is dominated by the negative AF exchange field due to the large $M_{s}$ of the sphere cluster to which the lone ion is coupled. This is the reason that $M_{I}$ is initially diamagnetic in Fig. (4.11). When $M_{s}$ becomes saturated, the exchange field $-\lambda M_{s}$ seen by a lone $\mathrm{Cu}$ ion no longer depends on $H$, and $H$ can become larger than the opposing exchange field, resulting in a paramagnetic $M_{I}$, as seen in Fig. (4.11) above $\approx 20 \mathrm{kG}$. We note that to obtain the diamagnetic behavior of the lone $\mathrm{Cu}$ ions below $20 \mathrm{kG}$ in Fig. 4.11, Eqs. (4.11) and (4.12) must be solved to at least second order.

The relation of the molecular field coupling constant $\lambda$ and conventional coupling constant $J$ in the spin Hamiltonian can be estimated by going to the high field limit, where the Brillouin function saturates to 1 . Then the maximum exchange field 


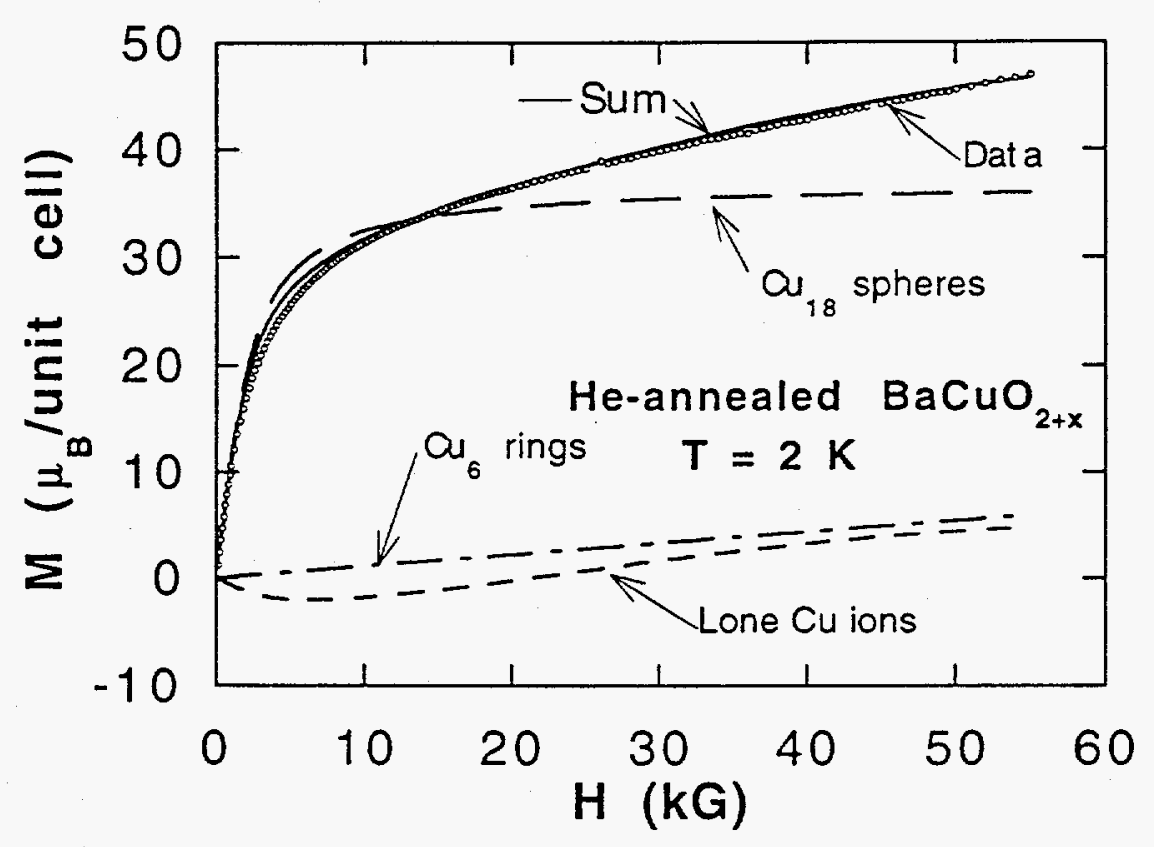

Figure 4.11: Magnetization $(M)$ versus applied magnetic field $(H)$ for He-annealed $\mathrm{BaCuO}_{2+x}$ (open circles). The solid curve labeled Sum is a theoretical fit to the data [Eq. (4.12)]. The contributions to the Sum from the lone $\mathrm{Cu}$ ions, the $\mathrm{Cu}_{6}$ clusters, and the $\mathrm{Cu}_{18}$ sphere clusters are as indicated. 
experienced by a lone ion is

$$
H_{e x, \max }=\lambda g S_{I} \mu_{B}=2.2 \times 10^{4} \text { Gauss. }
$$

This corresponds to an exchange energy

$$
E=g S_{s} \mu_{B} H_{e x, \max }=\lambda g^{2} S_{I} S_{s} \mu_{B}^{2}=1.5 \mathrm{~K} .
$$

If we convert this into the Heisenberg type interaction

$$
E=2 J \mathbf{S}_{s}^{i} \mathbf{S}_{I}^{i}
$$

we get

$$
2 J=2 \lambda g^{2} \mu_{B}^{2}=0.33 \mathrm{~K}=0.028 \mathrm{meV}
$$

\section{Low Temperature $\left(T \ll T_{N} \approx 15 \mathrm{~K}\right)$ Magnetic Susceptibility}

We write the magnetic susceptibility $\chi$ of a unit cell in a manner analogous to Eq. (4.4):

$$
\chi(T)=6 \chi_{I}(T)+2 \chi_{s}(T)+8 \chi_{r}(T)
$$

where $\chi_{I}, \chi_{s}$, and $\chi_{r}$ are respectively the susceptibilities of a lone $\mathrm{Cu}$ ion, a $\mathrm{Cu}_{18}$ sphere cluster and a $\mathrm{Cu}_{6}$ ring cluster. In the low temperature range $T \ll T_{N} \approx$ $15 \mathrm{~K}, \chi_{r}$ of the antiferromagnetically ordered $\mathrm{Cu}_{6}$ ring clusters should be nearly independent of $T$, and the $\mathrm{Cu}_{18}$ sphere clusters are in their ground states with spin $S_{s}=9$. The magnetic susceptibility $\chi$ is defined as $\chi=\lim _{H \rightarrow 0} M(H) / H$. In this low-field limit with $\mu_{B} H / \mathrm{k}_{\mathrm{B}} T \ll 1$, Eqs. (4.5) and (4.6) can be solved analytically [78] to yield the first two terms on the right-hand-side of Eq. (4.17):

$$
6 \chi_{I}(T)+2 \chi_{s}(T)=2 \frac{\left(C_{s}+3 C_{I}\right) T-6 \lambda C_{s} C_{I}}{T^{2}-3 \lambda^{2} C_{s} C_{I}}
$$


where $C_{s}$ and $C_{I}$ are respectively the Curie constants for a $\mathrm{Cu}_{18}$ sphere cluster ( $S_{s}$ $=9)$ and a lone $\mathrm{Cu}$ ion $\left(S_{I}=1 / 2\right)$. The right hand side of Eq. (4.18) has the same form as for a two-sublattice ferrimagnet [78].

Using the above values of $g=2.10$ and $\lambda=0.22 \mathrm{~N}_{A} \frac{G^{2}}{e r g}$, subtraction of $3 \chi_{I}(T)$ $+\chi_{s}(T)$ in the units appropriate to Fig. 4.12 from the observed $\chi$ yields, according to Eq. (4.17), the $\mathrm{Cu}_{6}$ ring susceptibility $\chi_{r}(T)$ which is shown in Fig. 4.12, along with $\left[6 \chi_{I}(T)+2 \chi_{s}(T)\right]^{-1}$. As independently deduced above in Fig. 4.5(bottom), $\chi_{r}(T)$ shows a maximum at $T_{N} \approx 13 \mathrm{~K}$ in Fig. 4.12 , indicative of long-range AF ordering of the $\mathrm{Cu}_{6}$ ring cluster magnetic moments below $T_{N}$. The extrapolated observed $\chi^{-1}$ data and predicted $\left[3 \chi_{I}(T)+\chi_{s}(T)\right]^{-1}$ data in Fig. 4.12 indicate that the $\mathrm{Cu}_{18}$ sphere clusters will order ferromagnetically below $\sim 1 \mathrm{~K}$.

High Temperature ( $\geq 40 \mathrm{~K}$ ) Susceptibility and Intracluster Coupling Constants

The Heisenberg model has been widely used to interpret the magnetic properties of the layered high $T_{c}$ cuprates. Similar to the $\mathrm{Cu}^{+2}$ ions in high $T_{c}$ cuprates, the $\mathrm{Cu}^{+2}$ ions in $\mathrm{BaCuO}_{2+x}$ are spin $1 / 2$ ions, as shown above. Therefore the exchange interaction between adjacent $\mathrm{Cu}$ ions should be nearly isotropic, and $\mathrm{BaCuO}_{2+x}$ should also be well described by the Heisenberg model. The magnetic susceptibility can be obtained in principle by diagonalizing the Heisenberg Hamiltonian

$$
\mathcal{H}=-2 J \sum_{<i, j>} \mathrm{s}_{i} \cdot \mathrm{s}_{j},
$$

as in the Bonner-Fisher [79] type of calculation, where $J>0$ is the nearest-neighbor $\mathrm{Cu}-\mathrm{Cu}$ FM exchange coupling constant and the sum is over nearest-neighbor $\mathrm{Cu}$ $\mathrm{Cu}$ pairs. However, due to limitations of computer memory we can only obtain the 


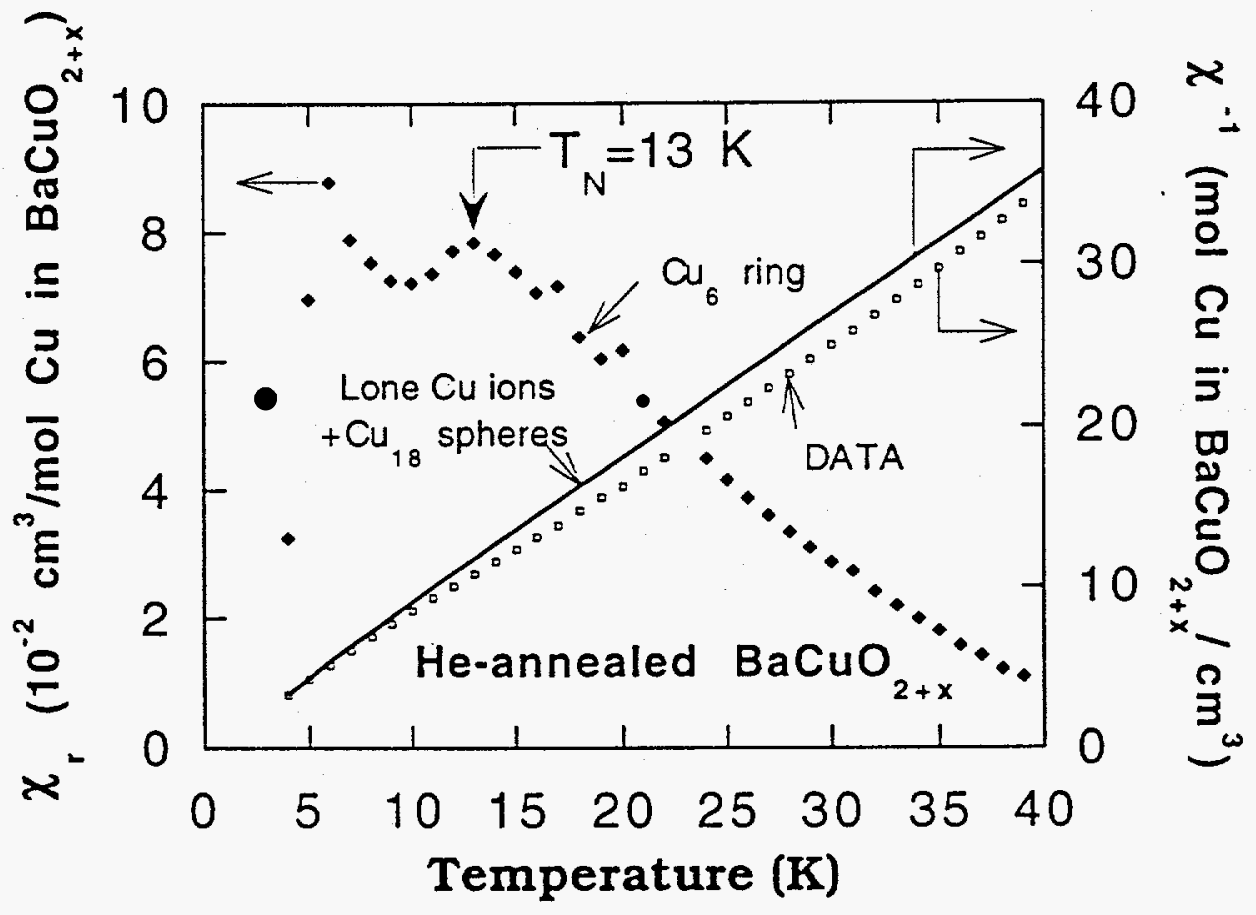

Figure 4.12: Inverse magnetic susceptibility $\chi^{-1}$ versus temperature $T$ for He-annealed $\mathrm{BaCuO}_{2+x}$ (open squares), reproduced from Fig. 4.4(Bottom). The solid curve is the predication of $\chi^{-1}$ for the lone $\mathrm{Cu}$ ions and $\mathrm{Cu}_{18}$ sphere clusters. The filled diamonds are the derived $\chi_{r}(T)$ data for the $\mathrm{Cu}_{6}$ rings. 
solution for up to an 11 spin cluster. Therefore we use Van Vleck's approximation [80] for the energy levels of a cluster:

$$
E_{S, \alpha}=-\frac{J_{\alpha} z_{\alpha}}{n_{\alpha}-1}\left[S(S+1)-n_{\alpha}{ }_{C u}\left(s_{C u}+1\right)\right],
$$

where $n_{\alpha}$ is the (even) number of $\mathrm{Cu}$ spins in the cluster, $S=0,1,2, \ldots, n_{\alpha} s_{C u}=$ $S_{\alpha, \max }$ is the spin of the cluster, $z_{\alpha}$ is the (average) number of nearest neighbors, ${ }^{s} C u=1 / 2$ is the spin of a copper ion, and $\alpha=s$ (sphere cluster) or $r$ (ring cluster). Here we assume that $J$ can be different within a sphere $\left(J_{s}\right)$ and within a ring $\left(J_{r}\right)$ cluster because of the different geometries of these two clusters. When the Zeeman energy $\mu_{B} H$ is much smaller than the thermal energy $\mathrm{k}_{\mathrm{B}} T$, the susceptibility of a cluster can be written as [77]

$$
\chi_{\alpha}(T)=\frac{\mathrm{N}_{\mathrm{A}} g^{2} \mu_{B}^{2}}{3 \mathrm{k}_{\mathrm{B}} T} \times \frac{\sum_{S=0}^{S=S_{\alpha, \max }} G_{S, \alpha} S(S+1)(2 S+1) e^{-E_{S, \alpha} / \mathrm{k}_{\mathrm{B}} T}}{\sum_{S=0}^{S=S_{\alpha, \max }} G_{S, \alpha}(2 S+1) e^{-E_{S, \alpha} / \mathrm{k}_{\mathrm{B}} T}},
$$

where $G_{S, \alpha}$ is the degeneracy of energy level $E_{S, \alpha}$, not counting the Zeeman degeneracy, and we assume $g_{s}=g_{r}=g$.

For the sphere cluster, $n_{s}=18, z_{s}=2.667$, and $G_{S, \alpha}=4862,11934,13260$, $9996,5508,2244,663,135,17$, and 1 for $S=0,1,2,3,4,5,6,7,8,9=S_{\text {sphere,max }}$, respectively. For the ring cluster, $n_{r}=6, z_{r}=2$, and $G_{S, r}=5,9,5$, and 1 for $S=$ $0,1,2,3=S_{\text {ring,max }}$, respectively. For a lone Cu ion, Eqs. (4.20) and (4.21) yield a Curie Law denoted by $\chi_{I}(T)$.

The total susceptibility $\chi(T)$ of a unit cell is given again by Eq. (4.17). Using Eqs. (4.20) and (4.21), the $1 / \chi(T)$ from Eq. (4.17) was fitted to our experimental data, using $g=2.10 J_{r}=148 \mathrm{~K}$, and $J_{s}=38 \mathrm{~K}$ as shown in Fig. 4.13(top).

The $\mathrm{Cu}_{6}$ ring cluster susceptibility $\chi_{r}$ can be calculated exactly using a BonnerFisher [79] type calculation. By comparing this susceptibility with that calculated 
using Eq. (4.21) and the approximate energy levels in Eq. (4.20), we may estimate the error in our $J_{r}$ and $J_{s}$ coupling constants derived above for the ring cluster, see Fig. 4.13(bottom), from which we conclude that our $J_{s}$ value has an error of less than $30 \mathrm{~K}$, which leads to $J_{r}=148 \pm 30 \mathrm{~K}$. If we assume that $J_{s}$ has a similar percentage error, one obtains $J_{s}=38 \pm 8 \mathrm{~K}$.

\section{Summary and Conclusions}

The motivation for studying the properties of magnetic clusters is interdisciplinary, in chemistry, biology, and physics [81]. The unique and complex structure of $\mathrm{BaCuO}_{2+x}$ makes this study very challenging but also very interesting. First the model of the magnetic structure and magnetic phase transition gives us insight about the magnetic interactions inside the clusters and between clusters, which is not only relevant to the mechanism of high $T_{c}$ superconductivity but also helps us to understand the quantum mechanics on a nanometer scale.

Our results show that the ring and the sphere clusters in $\mathrm{BaCuO}_{2+x}$ have ferromagnetic ground states with spin 3,9 respectively, where the FM intracluster interaction strengths are $148 \mathrm{~K}$ and $38 \mathrm{~K}$, respectively. This indicates that for nearly $90^{\circ} \mathrm{Cu}^{+2}-\mathrm{O}^{-2}-\mathrm{Cu}^{+2}$ bonds the superexchange interaction is ferromagnetic; this is relevant to many cuprate superconductors which show buckling of the $\mathrm{CuO}_{2}$ planes and significant deviations of the $\mathrm{Cu}^{+2}-\mathrm{O}^{-2}-\mathrm{Cu}^{+2}$ bond angle from $180^{\circ}$.

R. Gottschall and Robert Schöllhorn [82] have sucessfully made the barium nickel oxide with $\mathrm{BaCuO}_{2}$ type structure. Therefore substituting $\mathrm{Cu}$ by $\mathrm{Ni}$ and other metallic ions and studying how the magnetic properties change with the substitution will be very interesting. 

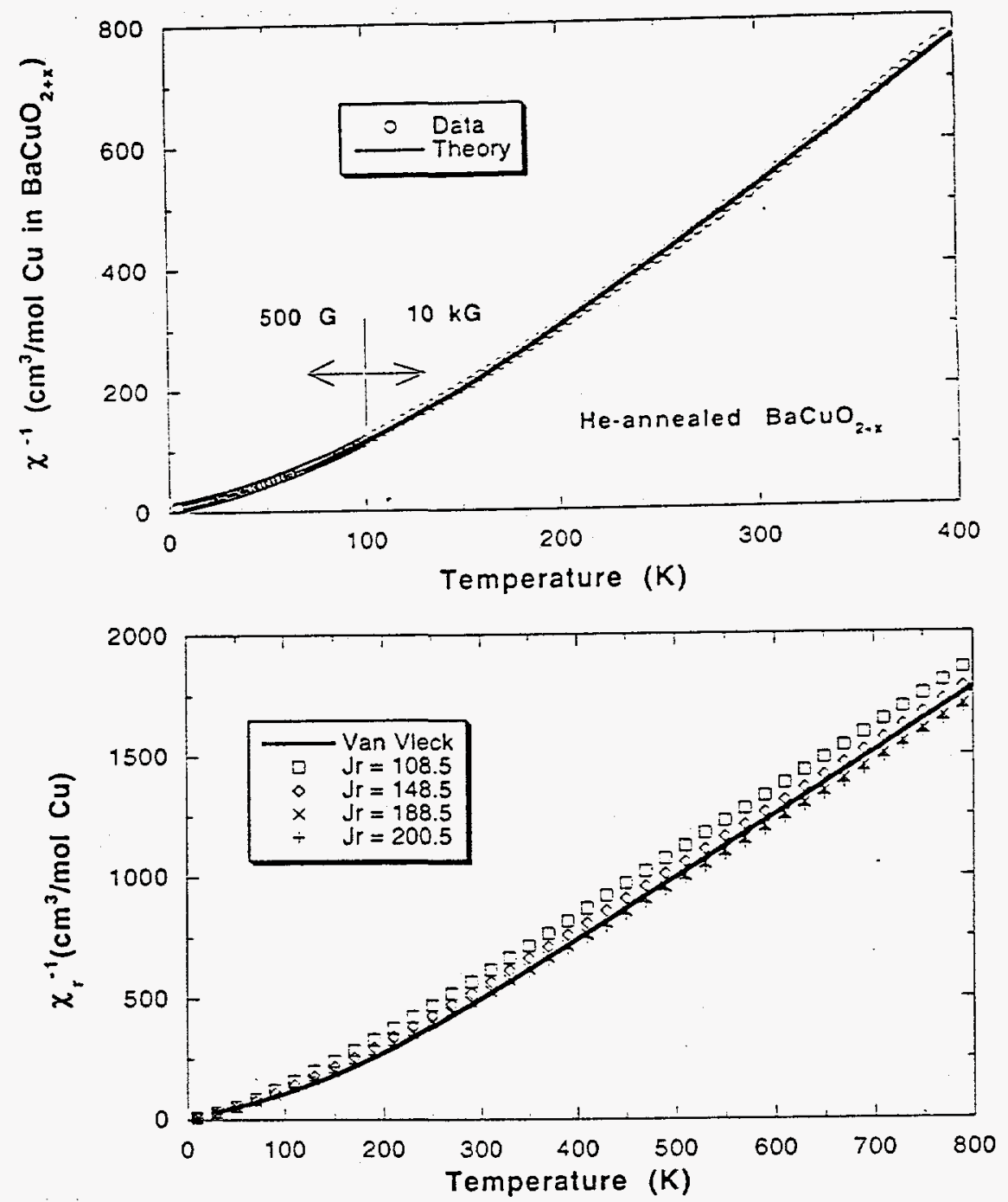

Figure 4.13: Top. Inverse magnetic susceptibility $\chi^{-1}$ versus temperature for He-annealed $\mathrm{BaCuO}_{2+x}$, reproduced from Fig. 4.4(Top). The solid curve is a theoretical fit [Eq. (4.16)] to the data above $70 \mathrm{~K}$. Bottom. Theoretical predictions for the inverse susceptibility $\chi^{-1}$ of the $\mathrm{Cu}_{6}$ rings versus temperature. The solid curve is the prediction using Eq. (4.21) and the approximate cluster magnetic energy levels in Eq. (4.20). The results of exact calculations using the Heisenberg model are shown by the various symbols for the corresponding values of exchange constant $J_{r}$. 


\section{Neutron Diffraction Study of Magnetic Ordering of Helium-annealed Sample}

Neutron diffraction measurements were carried out by D. Vaknin, J. FernandezBaca and X. L. Wang on the powder diffractometer HB-4, the triple axis spectrometer, HB1A and on the spin polarized triple-axis spectrometer HB-1, all at the High Flux Isotope Reactor (HFIR) at Oak Ridge National Laboratory. The 10g pellet-like polycrystalline sample was wrapped in a thin $\mathrm{Al}$ foil and loaded into an $\mathrm{Al}$ can under He atmosphere that was then mounted in various cryostats for different measurements.

The reflections allowed by the bcc crystal structure of $\mathrm{BaCuO}_{2+x}$ are of the type $h+k+1=$ even. At temperatures below $15 \mathrm{~K}$, extra reflections at the $\left(\begin{array}{ll}1 & 1\end{array}\right.$ 1) and at the $\left(\begin{array}{lll}2 & 2 & 1\end{array}\right)$ position are observed. Figure 4.14 shows a $2 \theta$ scan of the (1 $\left.\begin{array}{ll}1 & 1\end{array}\right)$ reflection at two different temperatures. The inset of Fig. 4.14 shows the temperature dependence of the integrated intensity of this reflection, with a clear indication that a phase transition occurs at $T=15.0 \pm 0.5 \mathrm{~K}$. Scans around the $\left(\begin{array}{lll}1 & 0 & 0\end{array}\right)$ and $\left(\begin{array}{lll}2 & 0 & 1\end{array}\right)$ reflections, with better statistics, did not show any appreciable intensity at $T=4.2 \mathrm{~K}$, and high order magnetic reflections were not identified due to the extensive overlap and dominance of nuclear reflections. High resolution neutron powder diffraction measurements above and below the transition temperature were performed and confirmed that no structural distortion occurs in the temperature range 4 - $16 \mathrm{~K}$, suggesting that the (1 111 ) reflection is associated with an AF long range order. Polarized beam measurements on the same sample proved unequivocally that the (llll) reflection is magnetic in origin [74], as shown in Fig. 4.15.

To model the magnetic neutron reflections we assumed that the long range order 


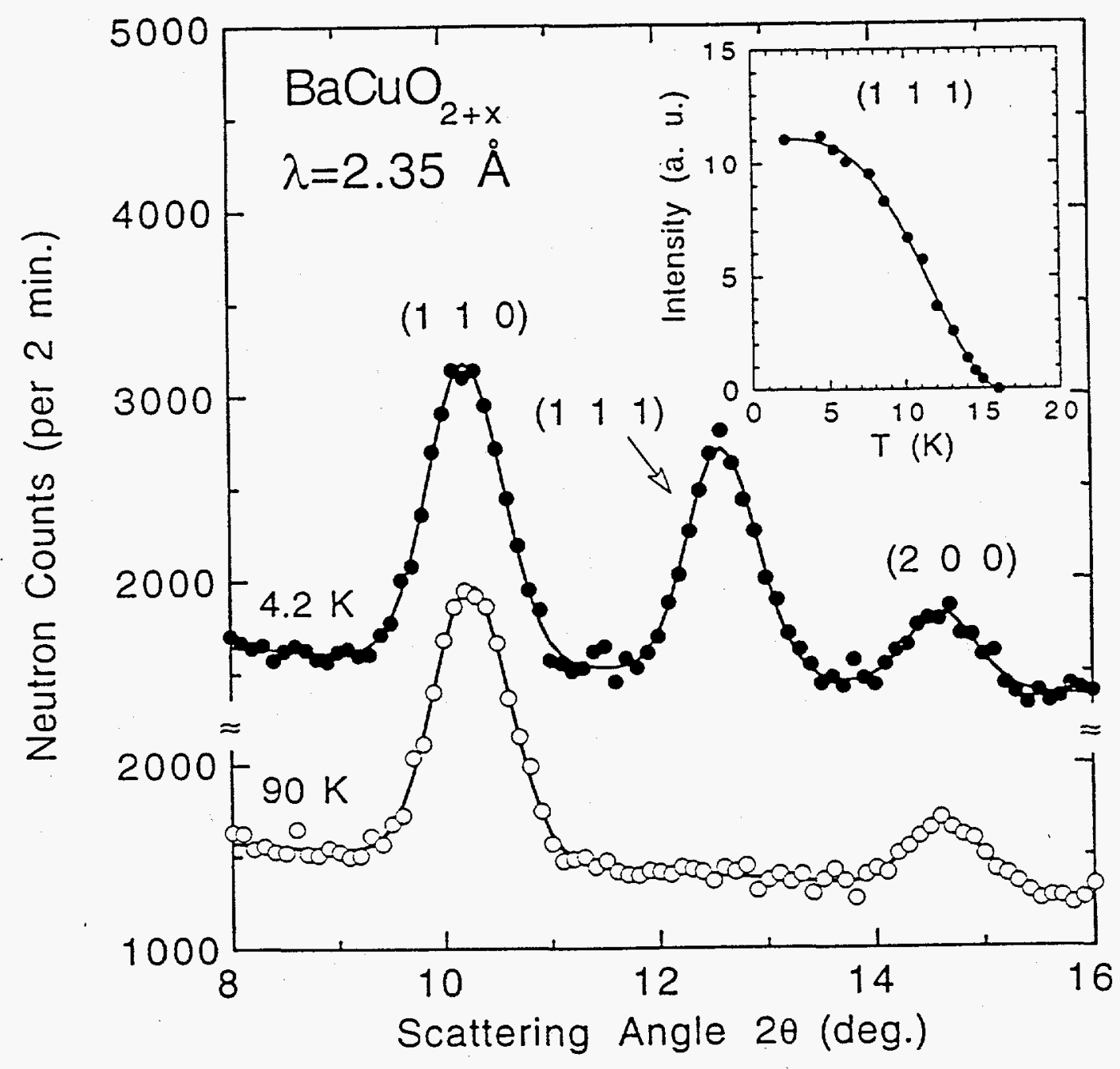

Figure 4.14: Unpolarized-neutron scans for the $(1,1,1)$ reflection at temperatures of $4.2 \mathrm{~K}$ and $90 \mathrm{~K}$. The solid curve for each scan is a multiple Gaussian fit to the experimental data. Inset: temperature dependence of the integrated intensity of the $(1,1,1)$ reflection (the solid curve is a guide to the eye). 
is primarily associated with the magnetic ring system, as discussed above. That is due to the general observation in model calculations that the $(1 / 41 / 41 / 4)$ position

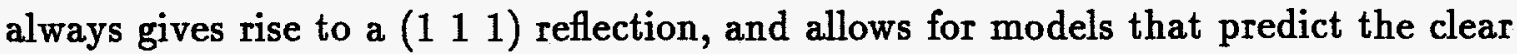
absence of the $\left(\begin{array}{lll}1 & 0 & 0\end{array}\right)$ reflection, whereas all AF model, incorporating the spheres predict prominent $\left(\begin{array}{lll}1 & 0 & 0\end{array}\right)$ and $\left(\begin{array}{lll}2 & 0 & 1\end{array}\right)$ reflections in contrast to the experimetal observations. The model that we proposed above is that the spins on each ring are internally ferromagnetically oriented and the spin on nearest neighbor $\mathrm{Cu}_{6}$ rings are antiparallel (powder diffraction measurements are insufficient for the determination of the actual direction of the moment [83]). The general form factor in this case is given by

$$
F=p_{0} \mu f(\mathbf{Q}) \sum_{i=1}^{8} F_{i} \sigma_{i} \exp \left(i \mathbf{r}_{i}^{0} \cdot \mathbf{Q}\right)
$$

where, $p_{0}=0.27 \times 10^{-12} \mathrm{~cm}, \mu$ and $f(\mathbf{Q})$ are, respectively, the magnetic moment and the magnetic form factor of an individual spin on the ring, $\sigma_{i}= \pm 1$, and $F_{i}$ is the structure factor of each ring given by

$$
F_{i}=\sum_{j=1}^{6} \exp \left(i \mathbf{r}_{i, j} \cdot \mathbf{Q}\right)
$$

where $\mathbf{r}_{i, j}$ are the relative positions of each spin with respect to the center point of the ring $\mathbf{r}_{i}^{0}$, so that the absolute position of spin $j$ in ring $i$ from the origin of the unit cell is given by $\mathbf{r}_{i}^{0}+\mathbf{r}_{i, j}$. The $\mathbf{r}_{i, j}$ 's are of three types $( \pm \epsilon, \pm \epsilon, 0),(0, \pm \epsilon, \pm \epsilon)$ and $( \pm \epsilon, 0, \pm \epsilon)$ where $\epsilon \approx 0.1008$. The plus or minus sign in the brackets should be chosen so that each $\mathbf{r}_{i, j}$ is orthogonal to the axis of highest symmetry of a ring. For instance, for the ring whose center is located at the $(1 / 41 / 4-1 / 4)$ position, the $\mathbf{r}_{i, j}$ 's are $(\epsilon,-\epsilon, 0),(\epsilon, \epsilon, 0),(0, \epsilon, \epsilon),(0,-\epsilon,-\epsilon),(\epsilon, 0,-\epsilon)$ and $(-\epsilon, 0, \epsilon)$. Each pair of $\mathrm{Cu}^{2+}$ rings sharing the same body diagonal are related by inversion symmetry 
Table 4.1: Observed and calculated integrated intensities of the the magnetic reflections at $\mathrm{T}=4.2 \mathrm{~K}$. The intensities of the magnetic reflections were normalized to a nuclear diffraction pattern of the angular $2 \theta$ range 8 60 degrees, excluding the magnetic reflections. The magnetic moment determined from the model described in the text is $\mu=0.89 \pm 0.05 \mu_{B}$.

\begin{tabular}{|c|c|c|c|}
\hline reflection & $\overline{\sin \theta / \lambda \AA^{-1}}$ & Calculated intensity & Integrated Intensity \\
\hline$\left(\begin{array}{lll}1 & 0 & 0\end{array}\right)$ & 0.0274 & forbidden & $*$ \\
\hline$\left(\begin{array}{lll}1 & 1 & 1\end{array}\right)$ & 0.0475 & 938 & $930 \pm 36$ \\
\hline$\left(\begin{array}{lll}2 & 0 & 1\end{array}\right)$ & 0.0613 & forbidden & $*$ \\
\hline$\left(\begin{array}{lll}2 & 2 & 1\end{array}\right)$ & 0.0823 & 148 & $137 \pm 16$ \\
\hline$\left(\begin{array}{lll}3 & 1 & 1\end{array}\right)$ & 0.0910 & 22 & $*$ \\
\hline$\left(\begin{array}{lll}3 & 2 & 2\end{array}\right)$ & 0.1131 & 72 & $*$ \\
\hline$\left(\begin{array}{lll}4 & 0 & 1\end{array}\right)$ & & forbidden & * \\
\hline$\left(\begin{array}{lll}4 & 2 & 1\end{array}\right)$ & 0.1257 & 40 & $*$ \\
\hline
\end{tabular}

and therfore have the same $F_{i}$. The general structure factor of any reflection can be readily calculated,

$$
\begin{aligned}
F= & 2 p_{0} \mu f(\mathbf{Q}) \cdot i \cdot \sin \left[\frac{\pi}{2}(h+k+l)\right] \\
& \cdot\left[F_{1}-F_{2} \exp (i \pi h)-F_{3} \exp (i \pi k)-F_{4} \exp (i \pi l)\right]
\end{aligned}
$$

Due to the $\sin \left[\frac{\pi}{2}(h+k+l)\right]$ factor, only (odd, odd, odd) and (even, even, odd) type of reflections are allowed. For the former case we get

$$
F=16 p_{0} \mu f(\mathbf{Q}) \cdot\{\cos [2 \pi h \epsilon] \cos [2 \pi k \epsilon]+\cos [2 \pi k \epsilon] \cos [2 \pi l \epsilon]+\cos [2 \pi l \epsilon] \cos [2 \pi h \epsilon]\}
$$

so that when $\epsilon=0, F$ reaches the maximum value of $48 p_{0} \mu f(\mathbf{Q})$. In Table 4.1 we list the calculated integrated intensities, $I=A \cdot M \cdot L \cdot F^{2}\left\langle\sin ^{2} \eta\right\rangle$, where $A$ is a scale factor obtained from the refinement of the nuclear reflections, $M$ the mutiplicity, and $\eta$ the angle between the magnetic moment and $\mathbf{Q}$. The average magnetic moment obtained from fitting our model [Eq. (4.24)] to the observed intensity yields a moment 


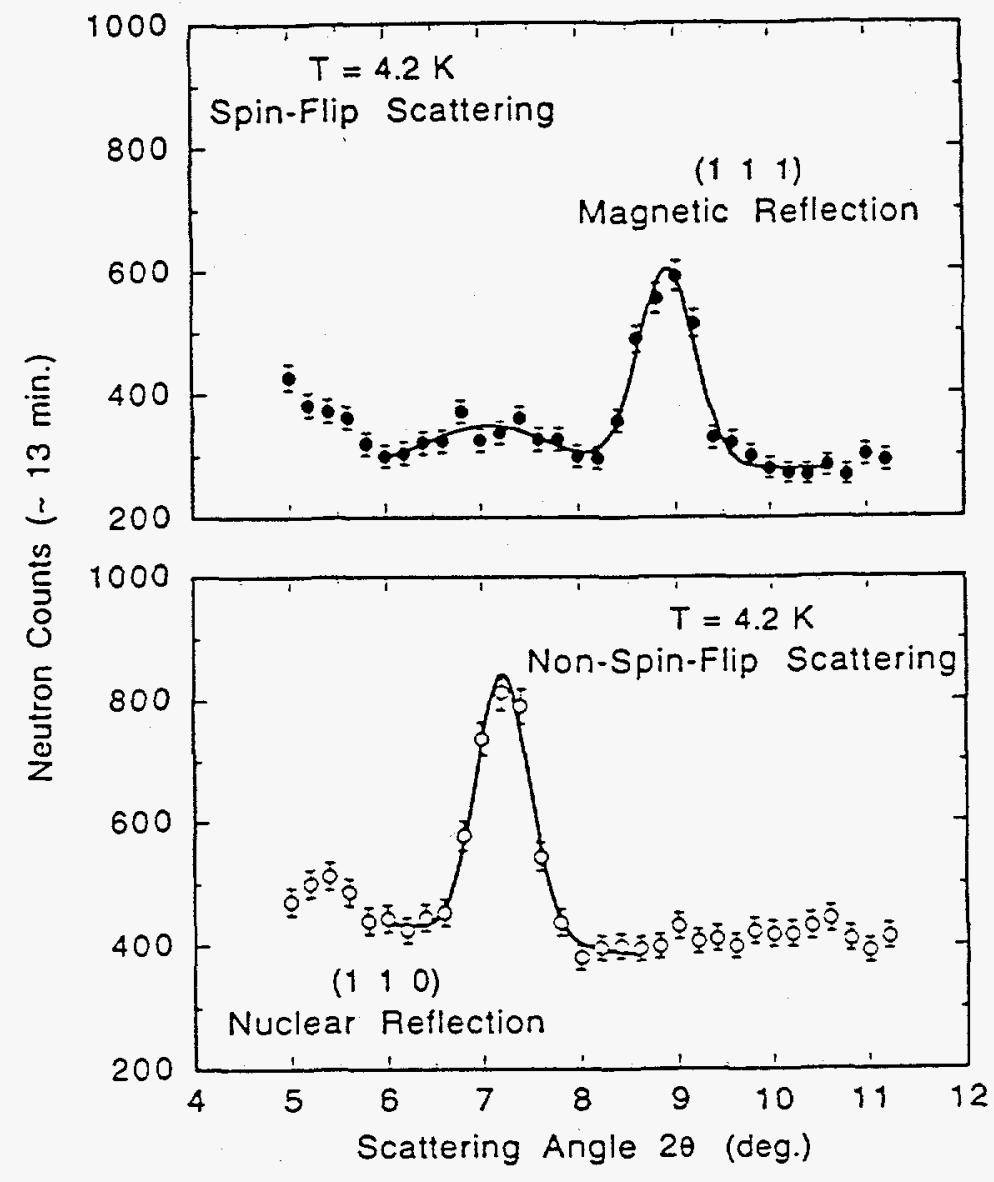

Figure 4.15: Polarized-neutron scans at $T=4.2 \mathrm{~K}$. The top and the bottom scans show the spin-flip (magnetic) and the non-spin-flip (nuclear) scattering, respectively. The solid curves are guides to the eye. 
$\mu=0.89 \pm 0.05 \mu_{B}$ using the form factor of localized $\mathrm{Cu}^{2+}$, Ref. [66]. The slightly reduced value of the magnetic moment from that expected $\left(1.1 \mu_{B}\right)$ for a free $\mathrm{Cu}^{2+}$ spin reinforces our belief that the sole contributors to the magnetic neutron scattering observations are the $\mathrm{Cu}_{6}$ ring clusters.

\section{Heat Capacity Study of Magnetic Ordering of Helium-annealed Sample}

The heat capacity $C$ for the He-annealed sample was measured by R. E. Fisher and coworkers at U. C. Berkeley at two different magnetic fields $H=0 G$ and $H=7$ $T$, as shown in Fig. 4.16. There is a clear heat capacity jump at the antiferromagnetic transition temperature $T_{N}=13 \mathrm{~K}$ for both $H=0 \mathrm{~T}$ and $H=7 \mathrm{~T}$. The value of the heat capacity jump at $T_{N}$ according to mean field theory is [84]

$$
\Delta C=5 K \frac{S(S+1)}{S^{2}+(S+1)^{2}}
$$

Where $\mathrm{S}$ is the total spin of the cluster. In our experimental $\Delta C$ is $1.2 \mathrm{~J} / \mathrm{K}$ mole, which is the same within $40 \%$ as the mean field value for the ring cluster assuming the spin on the cluster is $3 \mathrm{In}$ the low temperature range a plot of $\mathrm{C}$ versus $T$ (Fig. 4.17) shows is a maximum at about $0.8 \mathrm{~K}$, presumably from ferromagnetic ordering between the sphere clusters (above). 
92
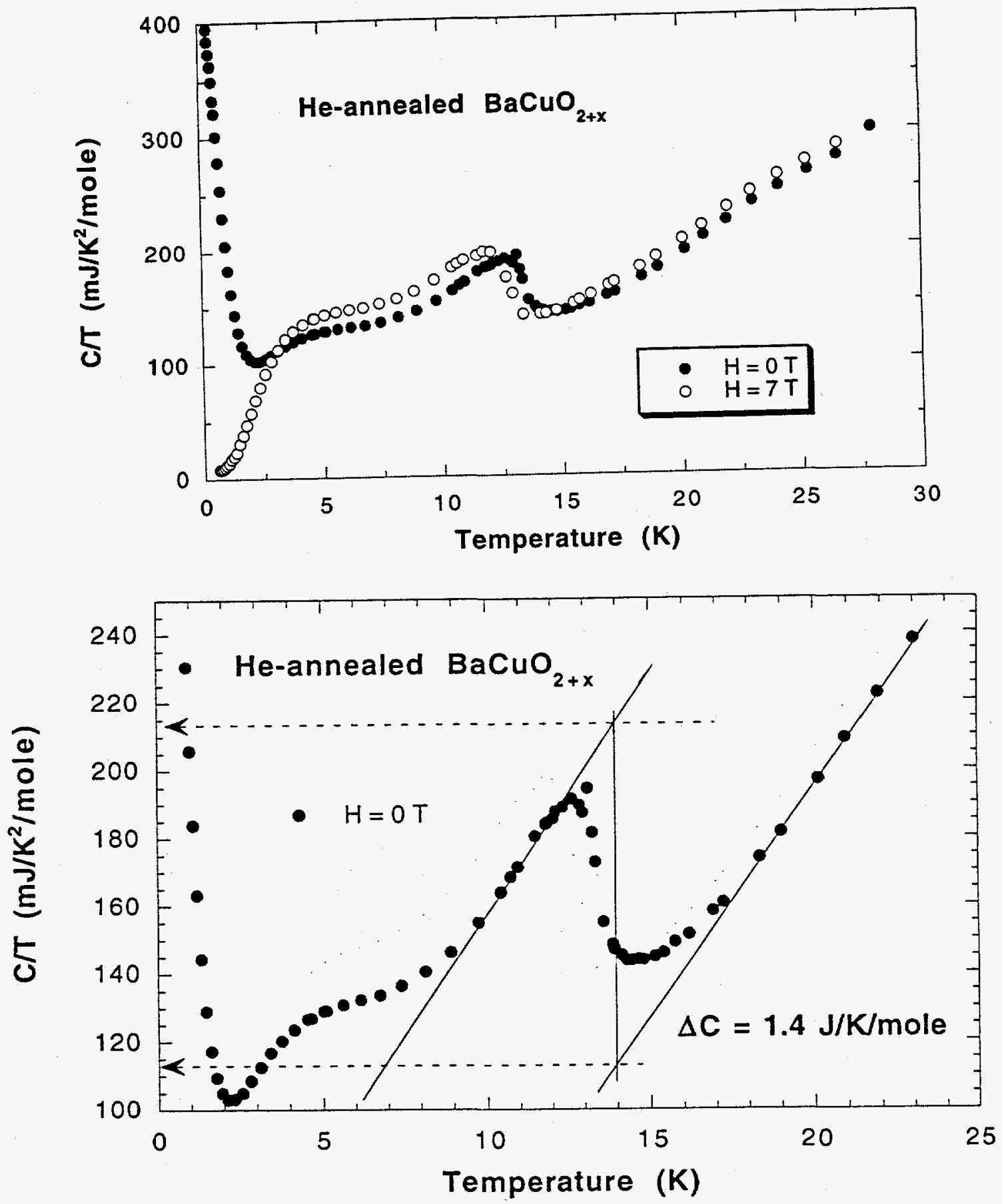

Figure 4.16: Heat capacity divided by temperature $C / T$, versus temperature. In the top panel, data were obtained in magnetic fields $H=0$ (open circles) and $70 \mathrm{kG}$ (filled circles). 

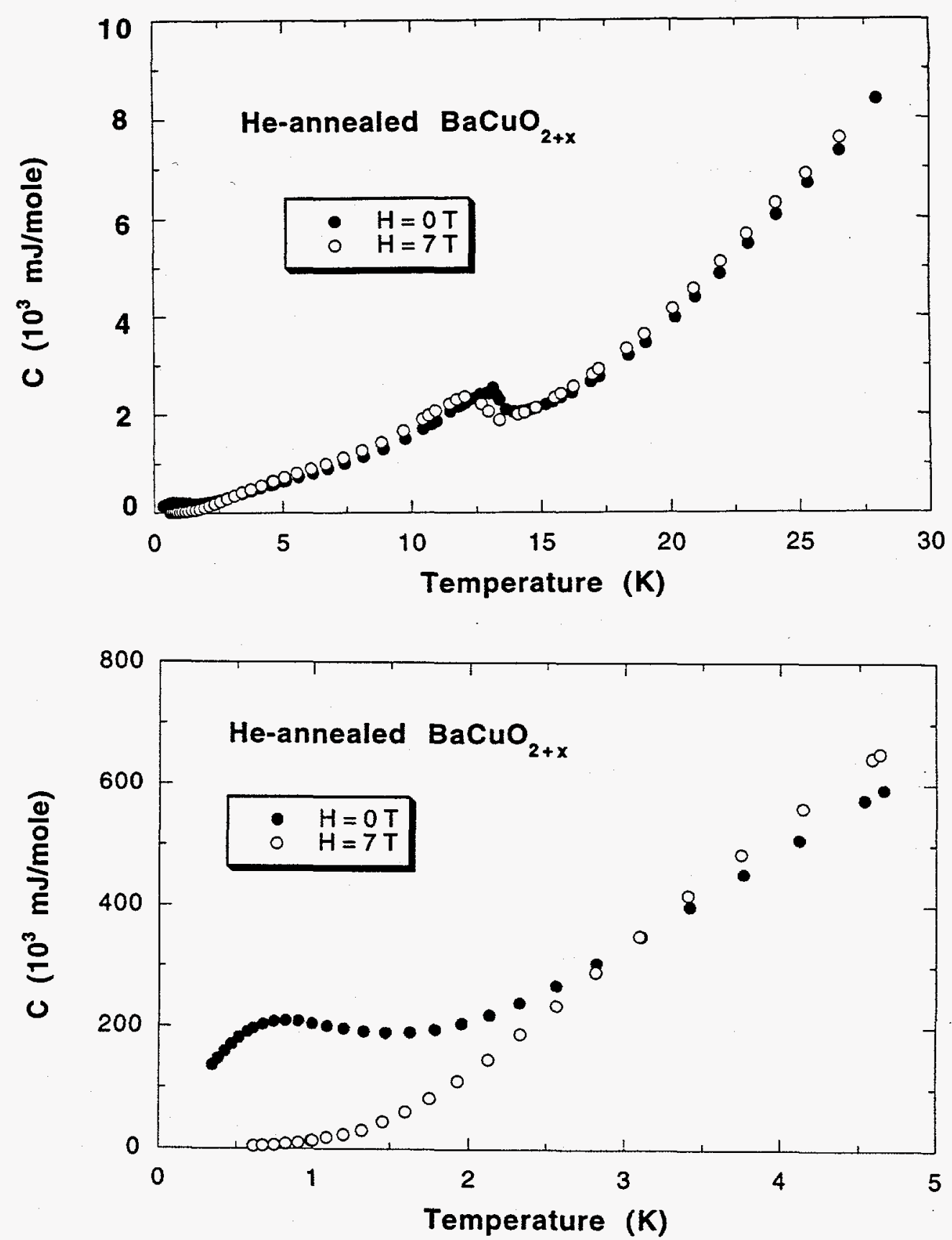

Figure 4.17: Heat capacity $C$ versus temperature for applied field $H=0$ (filled circles) and $H=7 \mathrm{~T}$ (open circles). 


\section{CHAPTER 5. MAGNETIC SUSCEPTIBILITY OF $\mathrm{Sr}_{2} \mathrm{CuO}_{3}$}

\section{Introduction}

Since the discovery of high temperature superconductivity, many studies have been performed both to search for new superconductors and elucidate the mechanism underlying the high transition temperature $\left(T_{c}\right)$. At this time, all known systems with $T_{c}>40 \mathrm{~K}$ possess 2 -dimensional $\mathrm{CuO}_{2}$ sheets which are generally agreed to play the active role in superconductivity. On the other hand, it has recently been reported [85] that $\mathrm{Sr}_{2} \mathrm{CuO}_{3+\delta}$ exhibits high temperature superconductivity for appropriate values of $\delta$. The parent insulating phase for these superconductors, $\mathrm{Sr}_{2} \mathrm{CuO}_{3}$, has a crystal structure [86] in which one dimensional $\mathrm{Cu}-\mathrm{O}$ chains, similar to those in $\mathrm{YBa}_{2} \mathrm{Cu}_{3} \mathrm{O}_{7}$, lie parallel to the a axis (Figure 5.1). The location(s) of the excess oxygen in $\mathrm{Sr}_{2} \mathrm{CuO}_{3+\delta}$ are not yet known, so it is not known whether this doped compound has an essentially quasi-one-dimensional or -two-dimensional structure. Although no one has reported superconductivity in materials which only have $\mathrm{Cu}$ $O$ one dimensional chains, the observation of superconductivity in Oxygen-doped $\mathrm{Sr}_{2} \mathrm{CuO}_{3+\delta}$ raises important questions concerning the minimum magnetic and electronic dimensionalities which will support high $T_{c}$. Furthermore, since the insulating parents of all other known high $T_{c}$ cuprate superconductors are two dimensional spin $1 / 2$ Heisenberg antiferromagnets [11], it is important to understand the magnetism 
in this one dimensional system as a prerequisite to determining its evolution as the system is doped into the metallic and superconducting state. Toward that end, we have studied the static magnetic properties of this material and observe direct evidence of Bonner-Fisher type behavior [79], indicating that $\mathrm{Sr}_{2} \mathrm{CuO}_{3}$ is a nearly ideal one-dimensional (1-D) spin 1/2 Heisenberg antiferromagnet.

\section{Experimental Details}

$\mathrm{Sr}_{2} \mathrm{CuO}_{3}$ was prepared by M. K. Crawford and Coworkers at DuPont using conventional solid state reaction. A one to one molar ratio of $\mathrm{SrCO}_{3}$ and $\mathrm{CuO}$ was mixed and fired at $950^{\circ}$ under flowing oxygen $(\sim 11 / \mathrm{min})$ for a total of 24 hours with one intermediate grinding. The calcined powders were pulverized, pelletized and sintered under the same conditions. Some samples were subjected to nitrogen treatment. Pellets were placed in an alumina boat and heated to between $400^{\circ} \mathrm{C}$ and $1000{ }^{\circ} \mathrm{C}$ under flowing nitrogen $(\sim 1 \mathrm{l} / \mathrm{min})$ for 12 hours. The decomposition temperature for this system is about $900{ }^{\circ} \mathrm{C}$ under the nitrogen atmosphere we employed. This nitrogen gas is obtained from liquid nitrogen boiloff and has a small residual oxygen partial pressure. We expect that the decomposition temperature of $\mathrm{Sr}_{2} \mathrm{CuO}_{3}$ would decrease below $900{ }^{\circ} \mathrm{C}$ if the oxygen partial pressure were further reduced and, in fact, samples heated above about $520{ }^{\circ} \mathrm{C}$ in 6 torr He in the Faraday magnetometer do decompose as shown in Fig. 5.2. The oxygen contents of the samples were determined iodometrically. Powder x-ray diffraction data for initial characterization were obtained with a Scintag diffractometer using $\mathrm{CuK} \alpha$ radiation at ambient temperature.

Magnetic susceptibilities below $300 \mathrm{~K}$ were measured using a SQUID magne- 


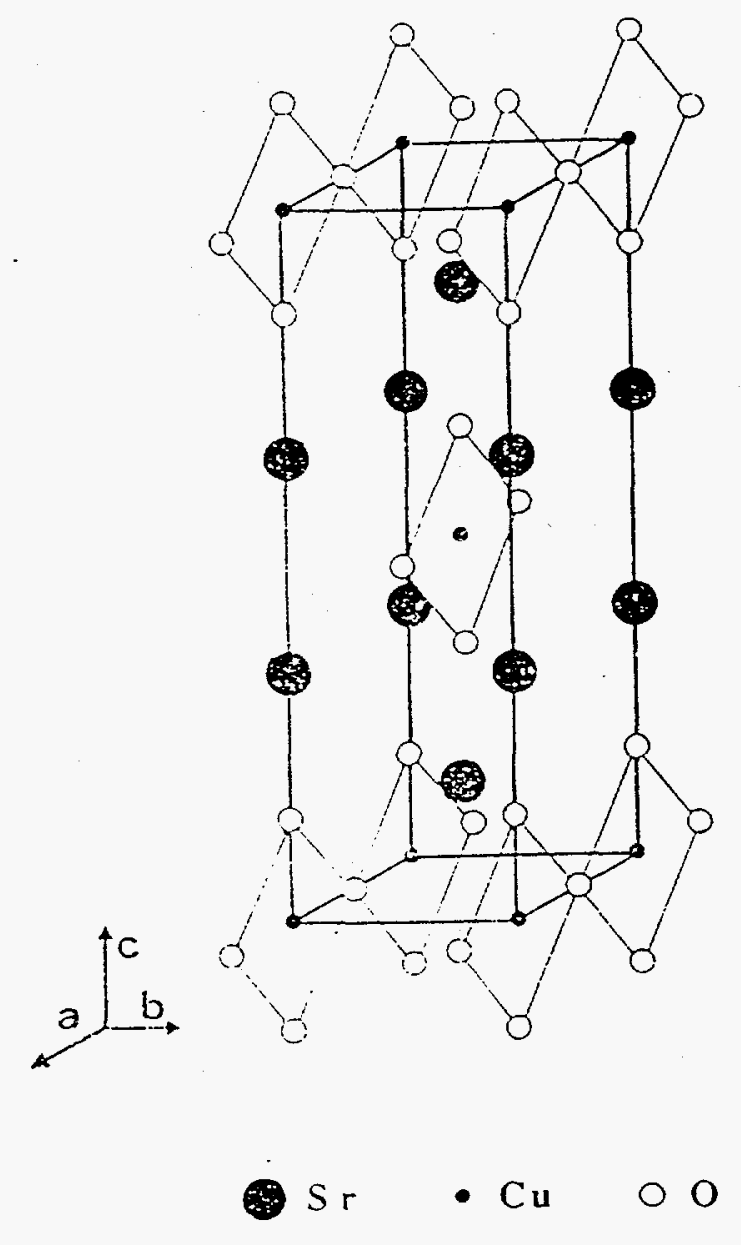

Figure 5.1: The crystal structure of $\mathrm{Sr}_{2} \mathrm{CuO}_{3}$. The $\mathrm{Cu}-\mathrm{O}$ chains are parallel to the a axis. 
Reversible $\mathrm{O}_{2}$ loss transition in Faraday Magnetometer

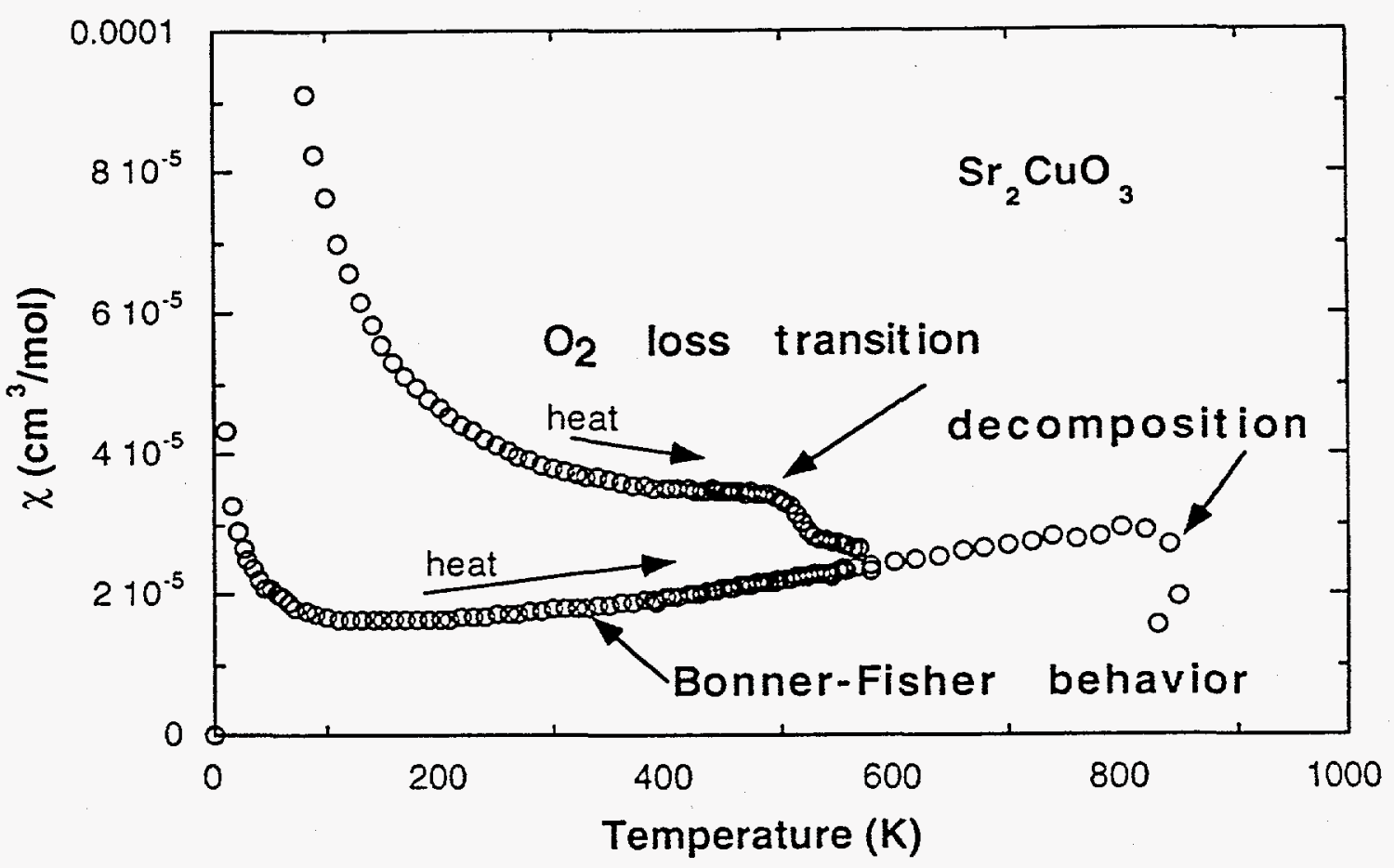

Figure 5.2: Magnetic susceptibility $\chi$ versus temperature for the first time running (upper curve) and second time running (lower curve). 
tometer (Quantum Design) at Dupont, whereas the high temperature susceptibility (to $800 \mathrm{~K}$ ) was measured using our Oxford Instruments Faraday balance. The sample holder was $99.999 \%$ pure gold wire. Its magnetic susceptibility was independently measured and substracted from the raw data for the $\mathrm{Sr}_{2} \mathrm{CuO}_{3 \pm \delta}$ samples. The contribution of ferromagnetic impurities to the measured magnetization was determined from magnetization-field isotherms between $25 \mathrm{~K}$ and $750 \mathrm{~K}$ (Fig. 5.3) and was found to correspond to that of 2 at. $\mathrm{ppm}$ or less, with respect to $\mathrm{Cu}$, of ferromagnetic iron metal impurities; this contribution is corrected for in Fig. 5.7 below. The magnetic measurements from the SQUID and the Faraday magnetometers were in good agreement below $300 \mathrm{~K}$.

Low temperature neutron powder diffraction studies were carried out at $11 \mathrm{~K}$ by the Dupont group using the BT-4 triple axis spectrometer at the reactor facility of the National Institute of Standard and Technology. The collimation was 20'-40'-10', and the neutron wavelength $(1.540 \AA)$ was chosen by a $\mathrm{Cu}(220)$ monochromator. The sample was mounted in an Al sample container containing He exchange gas. Low temperature synchrotron $\mathrm{x}$-ray powder diffraction [119] data were collected at $12 \mathrm{~K}$ by the Dupont group at beamline X-7A at the National Synchrotron Light Source at Brookhaven National Laboratory.

\section{Results}

\section{Synchrotron and Neutron Diffraction Studies}

The crystal structure of $\mathrm{Sr}_{2} \mathrm{CuO}_{3}$ at room temperature was first determined by single crystal $x$-ray [86] diffraction. Powder neutron [91] and $x$-ray diffraction [92] data at room temperature have also been reported. All of these studies assigned the 


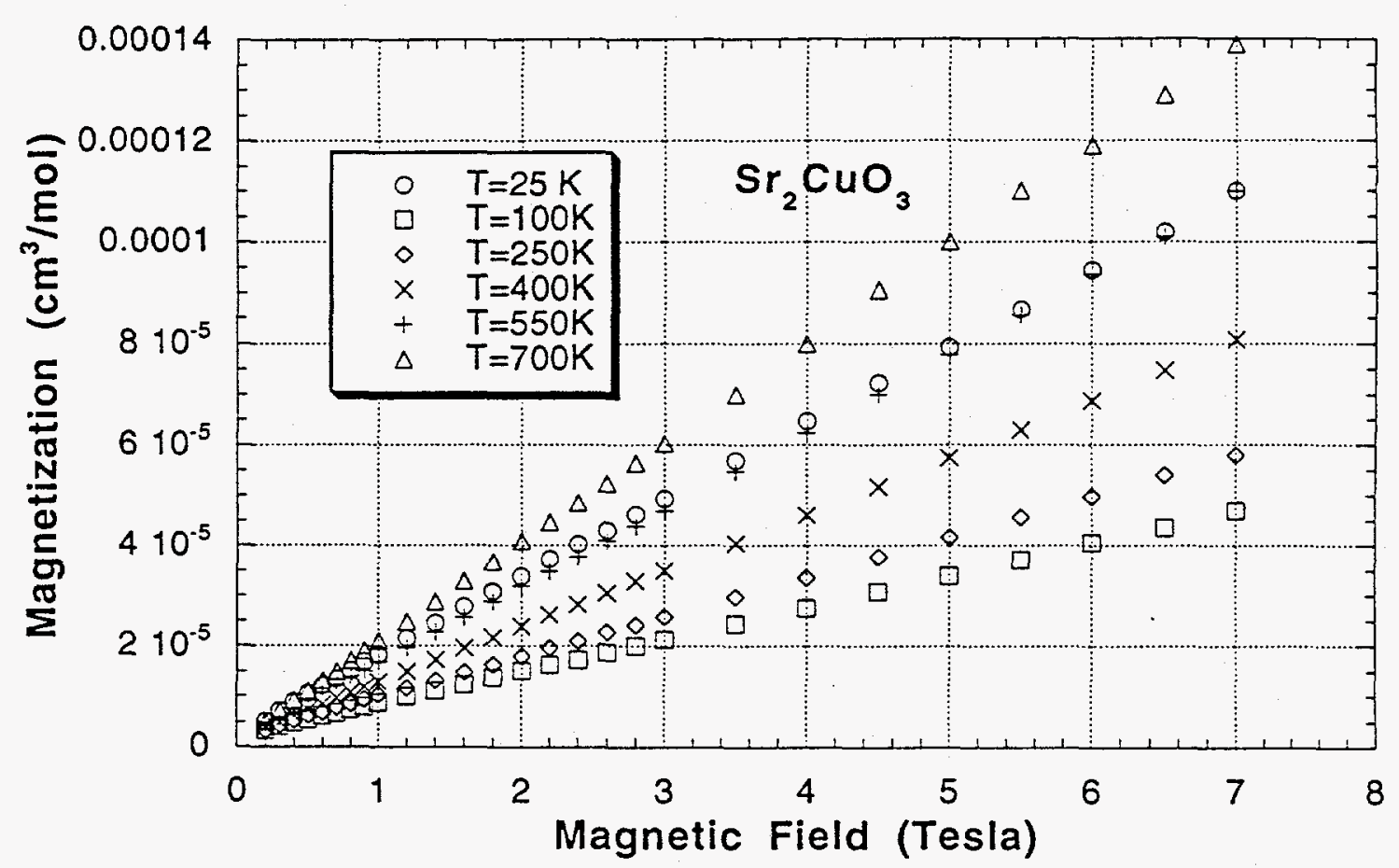

Figure 5.3: Magnetization versus magnetic field isotherms at different temperatures T. 
orthorhombic Immm space group to $\mathrm{Sr}_{2} \mathrm{CuO}_{3}$. We utilized neutron and synchrotron $\mathrm{x}$-ray powder diffraction at low temperature in order to search for any evidence of structural phase transitions or distortions which would further lower the symmetry and, for example, contribute to the magnetic anisotropy by allowing a DzyaloshinskyMoriya (D-M) interaction [93] to occur. It is known that the leading order magnetic anisotropy in several of the cuprates, for example $\mathrm{La}_{2} \mathrm{CuO}_{4}$, arises from the $\mathrm{D}-\mathrm{M}$ interaction.

Structural information determined by Rietveld refinement [94] of neutron diffraction data (Figure 5.4) obtained at $11 \mathrm{~K}$ are shown in Table 5.1. Aluminum reflections from the sample container used were excluded from the refinement. No superlattice peaks which would indicate lattice distortion were observed. High resolution synchrotron $\mathrm{X}$-ray diffraction data (Fig. 5.5 ) at $12 \mathrm{~K}$ yielded a similar conclusion. These results show that there are no structural transformations in $\mathrm{Sr}_{2} \mathrm{CuO}_{3}$ between room temperature and 11-12 $\mathrm{K}$, and thus suggest that the leading order magnetic anisotropy arises from magnetic dipole interactions and/or from anisotropy in the exchange coupling tensor.

\section{Magnetic Susceptibility}

There have been several previous studies [88]-[90] of the magnetic susceptibility of $\mathrm{Sr}_{2} \mathrm{CuO}_{3}$, but none of these studies observed clear evidence for Bonner-Fisher

[79] behavior, and therefore accurate values of $J$, the $\mathrm{Cu}^{+2}-\mathrm{Cu}^{+2}$ nearest-neighbor superexchange constant, were not determined. Furthermore, no systematic attempt was made in these earlier studies to account for the possible effect of oxygen nonstoichiometry on the magnetic susceptibilities. To address these problems, we have made 


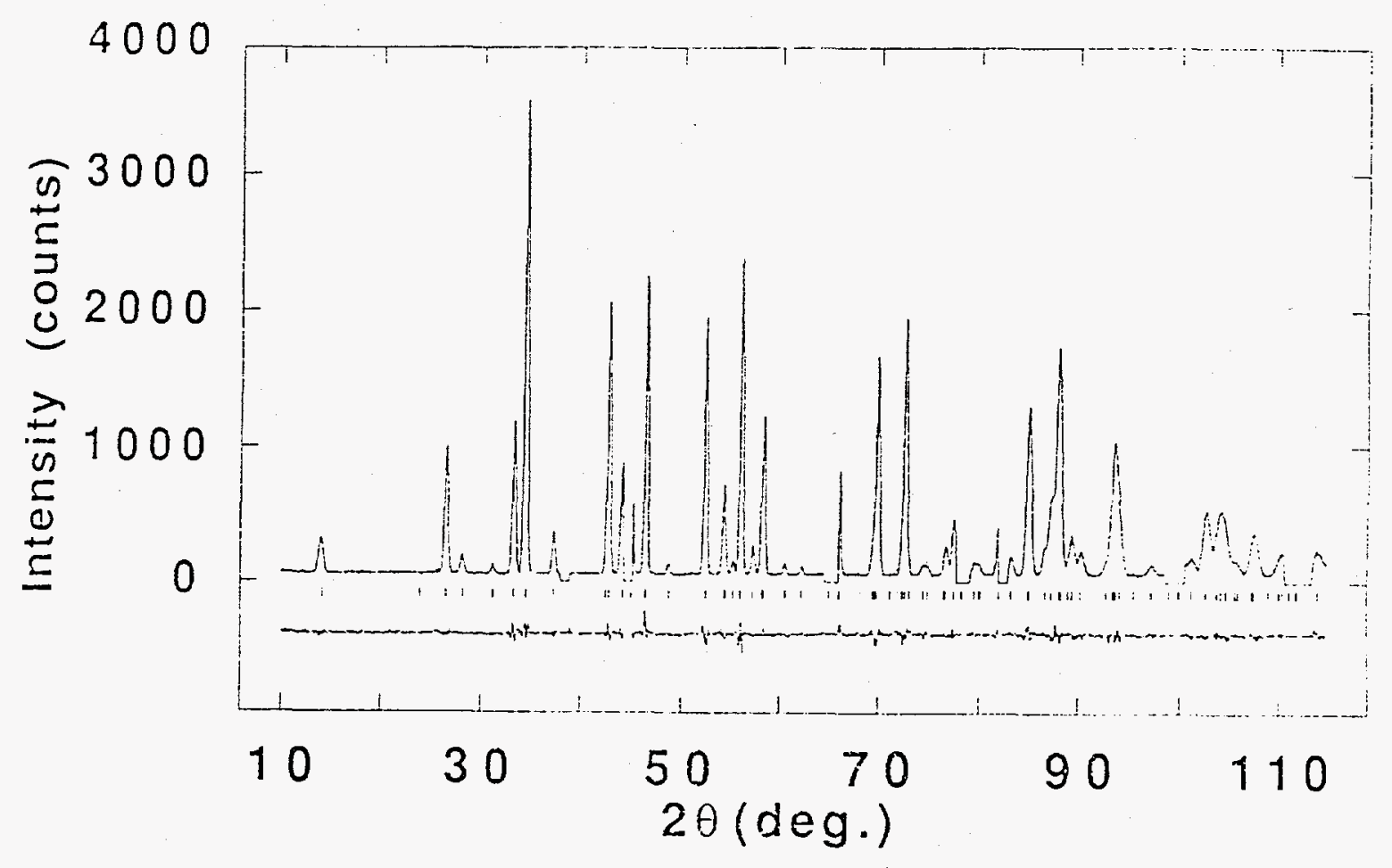

Figure 5.4: Powder neutron diffraction data collected at $11 \mathrm{~K}$. The results of Rietveld refinement are superimposed on the raw data. The vertical tick marks indicate the expected location of diffraction peaks. The fit residuals are plotted at the bottom of the figure. The neutron wavelength was $1.5401 \AA$. 


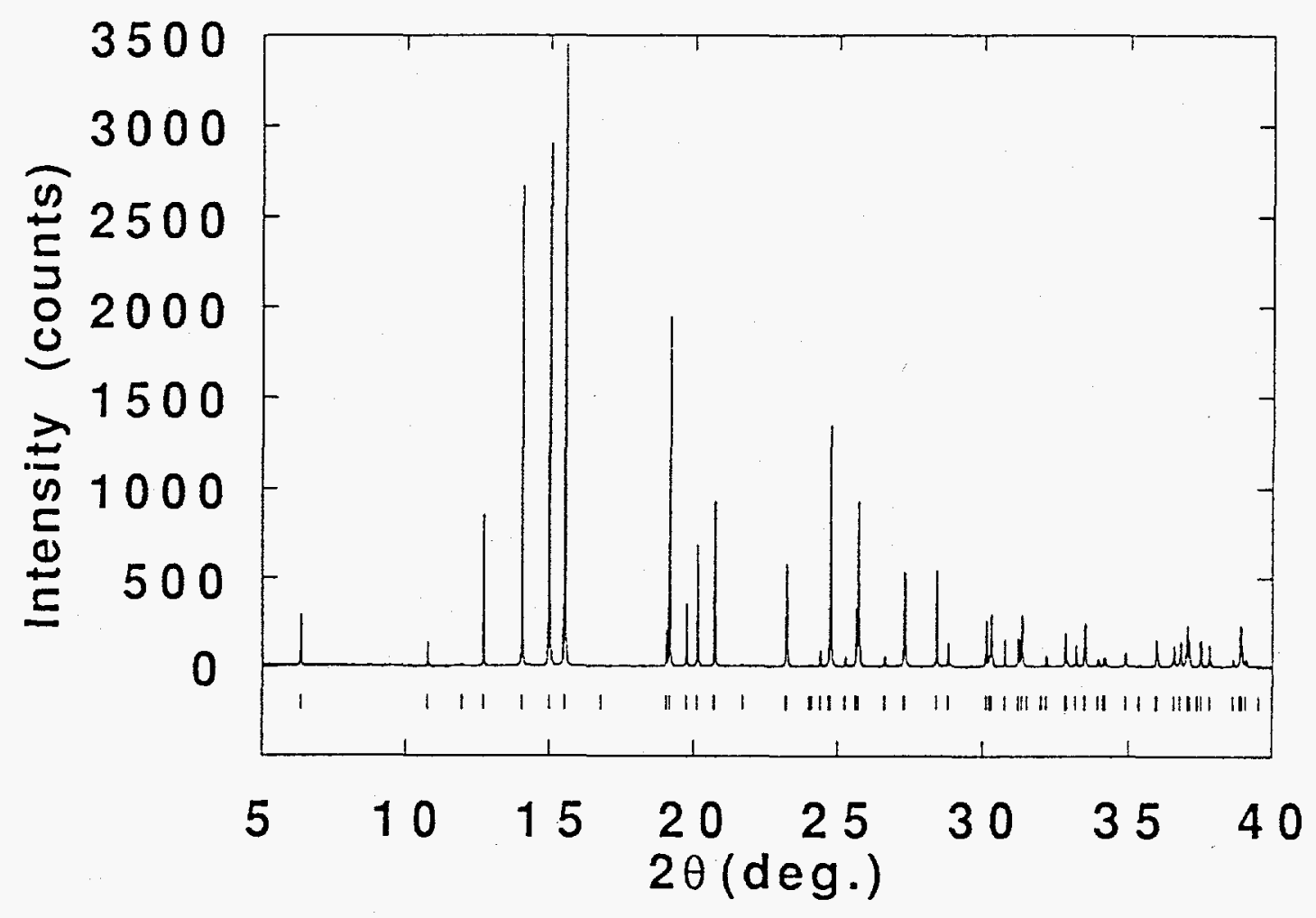

Figure 5.5: Synchrotron $\mathrm{x}$-ray diffraction data collected at $12 \mathrm{~K}$. The vertical tick marks indicate the expected location of diffraction peaks. The $\mathrm{x}$-ray wavelength was $0.70377 \AA$. 
Table 5.1: Low temperature crystallographic data for $\mathrm{Sr}_{2} \mathrm{CuO}_{3}$ determined by $\mathrm{Ri}$ etveld refinement of neutron diffraction data obtained at $11 \mathrm{~K}$.

\begin{tabular}{llllll}
\hline \hline Atom & $\mathrm{x}$ & $\mathrm{y}$ & $\mathrm{z}$ & $\mathrm{U}_{i s o}$ & Occupancy \\
$\mathrm{Sr}$ & 0 & 0 & $0.35195(9)$ & $0.006(4)$ & $0.980(4)$ \\
$\mathrm{Cu}$ & 0 & 0 & 0 & $0.001(5)$ & $0.990(6)$ \\
$\mathrm{O}(1)$ & 0 & 0 & $0.15445(11)$ & $0.0027(5)$ & $1.000(5)$ \\
$\mathrm{O}(2)$ & 0.5 & 0 & 0 & $0.0021(8)$ & $0.993(8)$ \\
\hline \hline
\end{tabular}

magnetic susceptibility measurements over a wide range of temperature on samples which have undergone various annealing treatments and therefore have slightly different oxygen contents (Table 5.2). The susceptibility is strongly affected by such treatments. Figure 5.6 shows the temperature dependencies below $300 \mathrm{~K}$ of the magnetic susceptibilities of samples before and after annealing under a $\mathrm{N}_{2}$ or a 6 torr He atmosphere. The diamagnetic terms originating in the closed shells of the ions have been subtracted $\left(\chi_{d i a}=-77 \times 10^{-6} \mathrm{~cm}^{3} /\right.$ mole). Plot (a) shows the susceptibility for an as-made sample before nitrogen treatment. As can be seen from this figure, treatment under a reducing atomosphere decreasing the susceptibility, especially the Curie-type component. Thus it is natural to associate the magnitude of this Curie-type term with the presence of excess oxygen in the lattice (this point will be discussed further below). In this picture, as-made sample (a) contains some excess oxygen ion defects, whereas nitrogen annealing reduces the number of such defects and consequently suppresses the Curie-Weiss-like behavior at low temperatures. Futhermore, Figure 5.6 shows the susceptibility for a sample kept at $600 \mathrm{~K}$ 
Table 5.2: $\quad$ Parameters for $\mathrm{Sr}_{2} \mathrm{CuO}_{3+\delta}$ derived from iodometric titrations and magnetic refinement assuming that $g=2.1,|J| / \mathrm{k}_{\mathrm{B}}=1307 \mathrm{~K}$.

\begin{tabular}{llllll}
\hline \hline (conditions) & O content $\delta$ & impurity level \% & $\theta(\mathrm{K})$ & $\chi_{V V}\left(\mathrm{~cm}^{3} / \mathrm{mole}\right)$ \\
(a) $950^{\circ} \mathrm{C}$ in $\mathrm{O}_{2}$ & $0.02(2)$ & 0.363 & -1.09 & 2.87 & $10^{-5}$ \\
(b) $600^{\circ} \mathrm{C}$ in $\mathrm{N}_{2}$ & $0.01(1)$ & 0.287 & -0.83 & 2.91 & $10^{-5}$ \\
(c) $800^{\circ} \mathrm{C}$ in $\mathrm{N} 2$ & $-0.01(1)$ & 0.227 & -0.86 & 2.63 & $10^{-5}$ \\
(d) $327^{\circ} \mathrm{C}$ in $6 \tau$ He no data & 0.114 & -7.81 & 2.28 & $10^{-5}$ \\
\hline \hline
\end{tabular}

under 6 torr He overnight and then measured without being removed from the $\mathrm{He}$ atmosphere. This sample shows a much smaller Curie-Weiss-type term compared with the other samples, presumably due to loss of almost all the excess oxygen after the overnight anneal. Since samples annealed in $\mathrm{N}_{2}$, but then exposed to air before the magnetic measurements were made, have large Curie-Weiss-type terms, this result also suggests that this material absorbs oxygen from air at room temperature fairly quickly, and that considerable care must be taken if one wishes to measure properties for the stoichiometric system.

Magnetic susceptibility $\chi$ measurements for $\mathrm{Sr}_{2} \mathrm{CuO}_{3 \pm \delta}$ made using the Faraday magnetometer in the temperature range of $2 \mathrm{~K}$ to $800 \mathrm{~K}$ also indicated a strong dependence upon oxygen content $\delta$, as shown above in Fig. 5.2. The as-made sample was first measured from $10 \mathrm{~K}$ to $600 \mathrm{~K}$. There is a clear decrease in $\chi$ at $540 \mathrm{~K}$, which indicates the loss of oxygen (oxygen loss at $540 \mathrm{~K}$ is confirmed by TGA in He at a flow rate of $40 \mathrm{cc} / \mathrm{min}$ ). Then the same sample was again measured from $10 \mathrm{~K}$ to 


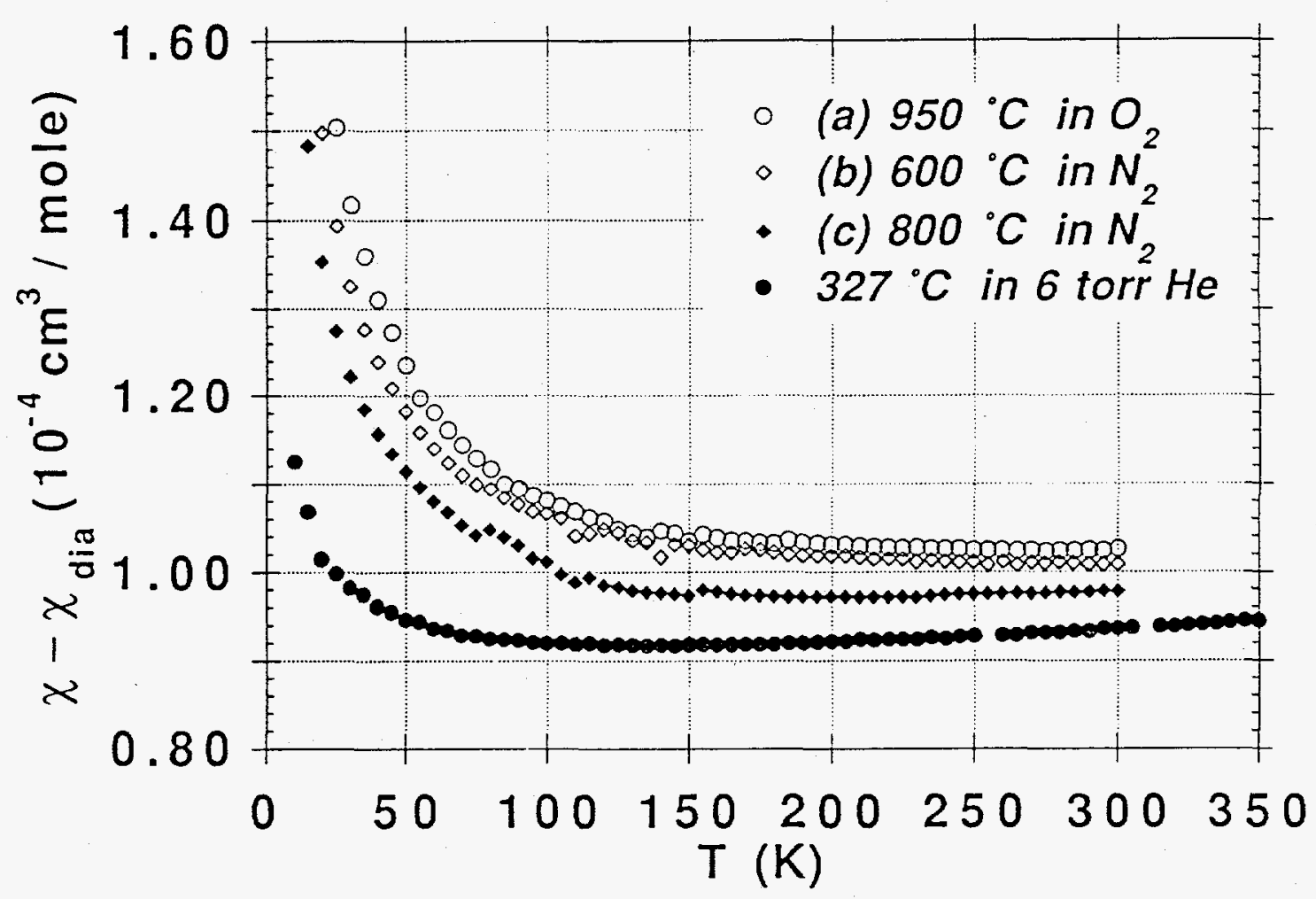

Figure 5.6: The temperature $(T)$ dependencies of the magnetic susceptibilities $(\chi)$ for the samples annealed under different conditions. The diamagnetic core contributions $\left(\chi_{d i a}\right)$ have been subtracted. The measurement field was $1.0 \mathrm{~T}$ in each case. 
$800 \mathrm{~K}$. Figure 5.2 shows a clear difference between these two curves.

Figure 5.7 shows the susceptibility versus temperature from 10 to $800 \mathrm{~K}$ for the sample annealed at $327{ }^{\circ} \mathrm{C}(600 \mathrm{~K})$ under 6 torr He then measured in the same atmosphere. $M(H)$ isotherms were measured between $25 \mathrm{~K}$ and $750 \mathrm{~K}$, and the susceptibility was found to be independent of applied magnetic field up to $H=7.0$ $\mathrm{T}$ (after correction for the influence of ferromagnetic impurities, see above). The enhancement of the susceptibility at low temperature presumably originates from a residual Curie-Weiss-like component, as mentioned above, but the gradual increase of the susceptibility with increasing temperature in the high temperature region is not Curie-Weiss like, but rather is similar to a Bonner-Fisher type susceptibility [79], as our fit to the data described below demonstrates.

\section{Data Analysis}

The magnetic Hamiltonian which describes a spin 1/2 Heisenberg chain,

$$
\mathcal{H}=2|J| \sum_{i} \overrightarrow{\mathrm{S}}_{i} \cdot \overrightarrow{\mathrm{s}}_{i+1}
$$

has been described previously [79]. In this expression, $J$ is the antiferromagnetic $\mathrm{Cu}^{+2}-\mathrm{Cu}^{+2}$ superexchange interaction coupling constant, and the summation extends over pairs of nearest neighbor spins. It is worthwhile spending a moment to justify the use of such a model to describe the magnetic susceptibility of $\mathrm{Sr}_{2} \mathrm{CuO}_{3}$. The assumption that $\mathrm{Sr}_{2} \mathrm{CuO}_{3}$ is a one dimensional magnetic system follows directly from the structure of this material (Fig. 5.1). Since the $\mathrm{Cu}^{+2}$ ions are only bonded to oxygen ions along the a direction with 180 degree $\mathrm{Cu}-\mathrm{O}-\mathrm{Cu}$ bonds, there will be strong $\mathrm{Cu}^{+2}-\mathrm{Cu}^{+2}$ antiferromagnetic superexchange interactions only in this direc- 
tion. The additional assumption that this is an Heisenberg system is based upon the fact that the $\mathrm{Cu}^{+2}$ ion is spin $1 / 2$ and thus has no single ion anisotropy to first order. Furthermore, the structural studies reported here and previously [91],[92] for $\mathrm{Sr}_{2} \mathrm{CuO}_{3}$ demonstrate that there is an inversion center located midway between the $\mathrm{Cu}^{+2}$ ions in the $\mathrm{Cu}-\mathrm{O}$ chains, which precludes the existence of a DzyaloshinskyMoriya (D-M) $\vec{S}_{i} \times \vec{S}_{j}$ interaction [93]. In contrast, $\mathrm{La}_{2} \mathrm{CuO}_{4}$, an antiferromagnetic insulator which is structurally similar to $\mathrm{Sr}_{2} \mathrm{CuO}_{3}$, has a leading order magnetic anisotropy arising from a D-M interaction which is allowed by the rotational distortion of the $\mathrm{CuO}_{6}$ octahedra in the $\mathrm{Bmab}$ (orthorhombic) phase [100]. We also expect that the interchain magnetic coupling will be rather weak in $\mathrm{Sr}_{2} \mathrm{CuO}_{3}$, partly as a result of magnetic frustration. For example, the interlayer interchain superexchange in $\mathrm{Sr}_{2} \mathrm{CuO}_{3}$ is fully frustrated despite the orthorhombic symmetry, again in contrast to the situation in orthorhombic $\mathrm{La}_{2} \mathrm{CuO}_{4}$ where the interlayer superexchange is not frustrated. This is true because, considering a given $\mathrm{Cu}^{+2}$ ion, the four nearestneighbor $\mathrm{Cu}^{+2}$ ions in the nearest neighbor chains in adjacent layers are equidistant in $\mathrm{Sr}_{2} \mathrm{CuO}_{3}$, whereas in $\mathrm{La}_{2} \mathrm{CuO}_{4}$ there are two such distances. In $\mathrm{Sr}_{2} \mathrm{CuO}_{3}$ the intralayer interchain interaction is, on the other hand, not frustrated, but is still expected to be rather weak due to the absence of intervening oxygen ions between adjacent $\mathrm{Cu}+2$ ions. Thus in general one expects $\mathrm{Sr}_{2} \mathrm{CuO}_{3}$ to be more Heisenberglike than $\mathrm{La}_{2} \mathrm{CuO}_{4}$, the latter nevertheless being considered one of the best examples of a spin 1/2 2-D Heisenberg antiferromagnet [101]. Therefore, we should expect $\mathrm{Sr}_{2} \mathrm{CuO}_{3}$ to be an excellent realization of a 1-D Heisenberg antiferromagnet and it seems very reasonable to attempt to describe its magnetic susceptibility with the Bonner-Fisher model for a spin 1/2 Heisenberg antiferromagnetic chain. 
In order to fit the susceptibility data shown in Fig. 5.7 over a wide temperature range, we assume that the observed temperature dependent magnetic susceptibility, $\chi(T)$, consist of several terms:

$$
\chi(T)=\chi_{d i a}+\chi_{V V}+\chi_{s p i n}(T)+\chi_{P a u l i}+\chi_{L a n d a u}
$$

In the absence of conduction electrons, the Pauli paramagnetism and Landau diamagnetism should be negligible. $\chi_{d i a}$ is the core diamagnetic susceptibility, $\chi_{V V}$ is the Van Vleck paramagnetic susceptibility, and $\chi_{s p i n}(T)$ is the spin susceptibility. The values of $\chi_{d i a}$ and $\chi_{V V}$ are assumed to be temperature-independent. The $\chi_{d i a}$ values used were found in standard tables [102]. Furthermore the spin susceptibility was separated into two parts:

$$
\chi_{\text {spin }}(T)=\rho \cdot\left(\chi_{C W}\right)+(1-\rho) \cdot\left(\chi_{B F}\right)
$$

where $\chi_{C W}$ is a Curie-Weiss-like component arising from finite length chains which consist of an odd number of $\mathrm{Cu}^{+2}$ spins [79], isolated $\mathrm{Cu}^{+2}$ spins, and/or other paramagnetic impurities, and $\chi_{B F}$ is the susceptibility of an $S=1 / 2$ infinite isotropic Heisenberg chain (the Bonner-Fisher susceptibility [79],[103]). The coefficient $\rho$ represents the defect/impurity level. There are no analytical solutions for the magnetic susceptibility of Heisenberg chains [79]. However, a useful closed-form approximation to $\chi_{B F}$ has been suggested [104] to be

$$
\chi_{B F}=\frac{N g^{2} \mu_{B}^{2}}{\mathrm{k}_{B} T} \frac{0.25+0.14995 X+0.30094 X^{2}}{1+1.9862 X+0.68854 X^{2}+6.0626 X^{3}}
$$

with $X=\frac{|J|}{k_{B} T}$. The Curie-Weiss-like term is

$$
\chi_{C W}=\frac{N g^{2} S(S+1) \mu_{B}^{2}}{3 \mathrm{k}_{B}(T-\theta)}
$$


Assuming that $g=2.1$, a typical value for cuprates [11],[105], and $S=1 / 2$, we determine the following parameters by fitting Eq. (5.3) to the magnetic susceptibility data in Fig. 5.7 after subtracting the core diamagnetism:

$$
\begin{gathered}
\left.\rho=1.14 \times 10^{-3} \text { (i.é, impurity level }=0.11 \%\right) \\
\theta=-7.81 \mathrm{~K} ; \\
|J| / \mathrm{k}_{B}=1307 \mathrm{~K} ; \\
\chi_{V V}=2.28 \times 10^{-5} \frac{\mathrm{cm}^{3}}{\mathrm{~mole}}
\end{gathered}
$$

The value of $\chi_{V V}$ is comparable to that of other single $\mathrm{Cu}-\mathrm{O}$ layer cuprates [106]. The fit using these parameter values is shown superimposed on the data in Fig. 5.7. The fit function has been extended to temperatures high enough to include the broad peak in the susceptibility arising from the appearance of short range 1-D order with decreasing temperature. Futhermore, several curves calculated assuming different values of $J$ are plotted to give a sense of the sensitivity of the fit to this important parameter. From these curves we estimate the value of $|J| / k_{B}$ to be $(1300 \pm 200) \mathrm{K}$.

As mentioned previously, a Curie-Weiss-type term can originate from several sources including nearly isolated $\mathrm{Cu}^{2+}$ ions associated with oxygen defects (oxygen vacancies or excess oxygen), some other kinds of lattice defects, or magnetic impurites. However, an equally plausible explanation is that the Curie-Weiss-like behavior comes from the random termination of $\mathrm{Cu}-\mathrm{O}$ chains. As Bonner and Fisher have discussed [79], if the resulting chains have an even number of $\mathrm{Cu}^{2+}$ spins, then the total spin on the chain in the antiferromagnetically ordered state is zero at $T=0$. If, however, the chains have an odd number of $\mathrm{Cu}^{2+}$ spins, then in the antiferromagnetically ordered state the net spin on the chain is $1 / 2$. Such chains of odd length 


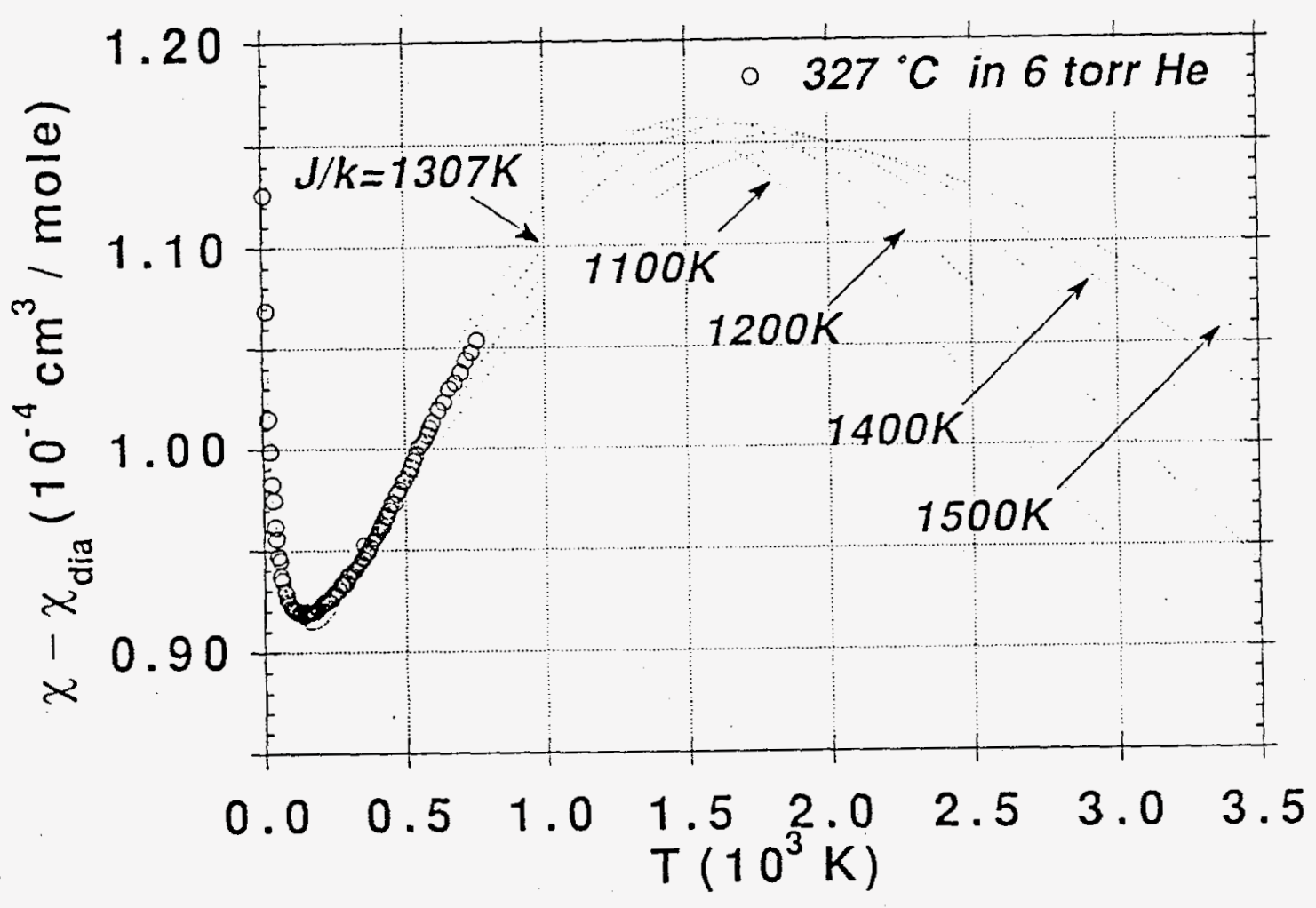

Figure 5.7: The magnetic susceptibility $\left(\chi-\chi_{d i a}\right)$ versus temperature up to $800 \mathrm{~K}$ for the sample annealed under 6 torr helium. The experimental data are represented by open circles, while the theoretical curves described in the text are shown by dotted lines. 
will make contributions to the magnetic susceptibility which are Curie-Weiss-like, although both the amplitude of the susceptibility divergence and the temperature at which the divergence occurs are depressed with increasing chain length by $\mathrm{Cu}^{2}+$ $\mathrm{Cu}^{2+}$ antiferromagnetic interactions. In the opposite limit, "chains" which have a length of only one $\mathrm{Cu}^{2+}$ ion will precisely exhibit Curie-Weiss behavior. To avoid the additional complications necessary to model contributions to the magnetic susceptibility arising from chains with a random distribution of lengths, we have chosen to represent the impurity spin susceptibility for $\mathrm{Sr}_{2} \mathrm{CuO}_{3}$ simply as a Curie-Weiss term which adequately represents the contributions from odd length finite chains, as well as additional contributions due to isolated $\mathrm{Cu}^{+2}$ ions and/or impurites. This simplification minimizes the number of refinement parameters, and the quality of the fit indicates it to be a reasonable approximation.

The results of these measurements thus indicate that $\mathrm{Sr}_{2} \mathrm{CuO}_{3}$ is an excellent realization of a 1-D spin 1/2 Heisenberg antiferromagnet. In fact, it may be the best realization of such a system reported to date. In order to place $\mathrm{Sr}_{2} \mathrm{CuO}_{3}$ in the context of other 1-D antiferromagnets, Table 5.3 shows parameters for several such systems which have appeared in the literature [99]. $J_{1}$ and $J_{2}$ are the two interchain magnetic coupling constants in orthogonal directions (in the $b$ and $c$ directions in $\mathrm{Sr}_{2} \mathrm{CuO}_{3}$ ). For all materials other than $\mathrm{Sr}_{2} \mathrm{CuO}_{3}$ in Table 5.3 we have assumed that $J_{1}=J_{2}$. We have also calculated independent values of $J_{1}$ and $J_{2}$ for $\mathrm{Sr}_{2} \mathrm{CuO}_{3}$, assuming only dipolar interaction coupling, using the expression

$$
J=-\sum_{i} \frac{\mu \cdot \mu_{\mathrm{i}}\left(3 \cos ^{2} \phi_{i}-1\right)}{r_{i}^{3}}
$$

where the sum is over the $\mathrm{Cu}$ moments $\mu_{\mathrm{i}}$ in the same $\left(J=J_{1}\right)$ or different $(J=$ 
Table 5.3: $\quad \mathrm{J}, \mathrm{J}_{1}, \mathrm{~J}_{2}$ and $\mathrm{T}_{N}$ values for several linear chain antiferromagnets compared with the values for $\mathrm{Sr}_{2} \mathrm{CuO}_{3}$. Note that $J_{1}$ and $\mathrm{J}_{2}$ for $\mathrm{Sr}_{2} \mathrm{CuO}_{3}$ are the values estimated assuming only dipolar coupling between the $\mathrm{Cu}^{2+}$ spins.

\begin{tabular}{lclllll}
\hline \hline & $\mathrm{T}_{N}(\mathrm{~K})$ & $\mathrm{J}(\mathrm{K})$ & $\mathrm{S}(\mathrm{spin})$ & $\mathrm{I}\left(\eta_{1}, \eta_{2}\right)$ & $\eta_{1}, \eta_{2}$ & $\mathrm{~J}_{1}(\mathrm{~K}), \mathrm{J}_{2}(\mathrm{~K})$ \\
$\mathrm{Sr}_{2} \mathrm{CuO}_{3}$ & 0.028 & 1307 & $1 / 2$ & $4.7 \times 10^{4}$ & $2.8 \times 10^{-5}$, & 0.036, \\
& & & & & $5.1 \times 10^{-7}$ & $6.6 \times 10^{-4}$ \\
$\mathrm{Sr}_{2} \mathrm{CuO}_{3}$ & 5 & 1307 & $1 / 2$ & 260 & $6.06 \times 10^{-6}$ & $7.88 \times 10^{-3}$ \\
$\mathrm{TMMC}$ & 0.84 & 6.5 & $5 / 2$ & 90.3 & $5.03 \times 10^{-5}$ & $3.27 \times 10^{-4}$ \\
$\mathrm{BrMnBr}_{3}$ & 8.8 & 12 & $5 / 2$ & 15.9 & $1.62 \times 10^{-3}$ & $1.94 \times 10^{-2}$ \\
$\mathrm{CsMnBr}_{3}$ & 8.3 & 9.6 & $5 / 2$ & 13.5 & $2.25 \times 10^{-3}$ & $2.16 \times 10^{-2}$ \\
$\mathrm{CuCl}_{2} \cdot 2 \mathrm{NC}_{5} \mathrm{H}_{5} 1.7$ & 13 & $1 / 2$ & 7.65 & $7.00 \times 10^{-3}$ & $9.10 \times 10^{-2}$ \\
$\mathrm{CsNiCl}_{3}$ & 4.5 & 11 & 1.0 & 6.52 & $9.64 \times 10^{-3}$ & $1.06 \times 10^{-1}$ \\
$\mathrm{KCuF}_{3}$ & 39.8 & 203 & $1 / 2$ & 6.40 & $1.42 \times 10^{-2}$ & 3.2 \\
$\mathrm{KCuF}_{3}$ & & & & & $1.0 \times 10^{-2}$ & 1.9 \\
$\mathrm{RbNiCl}_{3}$ & 11 & 11 & 1.0 & 2.67 & $5.67 \times 10^{-2}$ & 0.634 \\
\hline \hline
\end{tabular}


$J_{2}$ ) layers, $\mathrm{r}_{i}$ is the distance between the moment $\mu$ and the moment $\mu_{i}$, and $\phi_{i}$ is the angle between $r_{i}$ and the $a$ axis. For this calculation the magnetic structure was assumed to be the same as that reported for $\mathrm{Sr}_{2} \mathrm{CuO}_{2} \mathrm{Cl}_{2}$ [109]. The summation in Eq. (5.10) included $\mathrm{Cu}^{+2}$ moments within a distance $\mathrm{r}_{i}$ sufficient to achieve convergence.

Values of $J_{1}\left(=J_{2}\right)$ in Table 5.3 were calculated from the following expressions [110]-[112]

$$
\frac{k T_{N}}{|J|}=\frac{4 S(S+1) / 3}{I\left(\eta_{1}, \eta_{2}\right)}
$$

where $\eta_{1}=\mathrm{J}_{1} / \mathrm{J}, \eta_{2}=\mathrm{J}_{2} / \mathrm{J}$, and

$$
I\left(\eta_{1}, \eta_{2}\right)=\frac{1}{\pi^{3}} \iiint_{0}^{\pi} \frac{d q_{x} d q_{y} d q_{z}}{\eta_{1}\left(1-\cos q_{x}\right)+\eta_{2}\left(1-\cos q_{y}\right)+\left(1-\cos q_{z}\right)}
$$

For the case where $J / J_{1} \gg 1$, and $J_{1} / J_{2} \geq 1$, Eq. 5.12 can be approximated by $[112]$

$$
I\left(\eta_{1}, \eta_{2}\right)=\frac{0.64}{\sqrt{\eta_{1}}}\left[1+0.253 \ln \left(\frac{\eta_{1}}{\eta_{2}}\right)\right] .
$$

For $J / J_{1} \geq 10$, and $J_{1} / J_{2} \geq 10$, we find that Eq. (5.13) is accurate to better than $5 \%$. Thus the interchain coupling energies can be estimated using the experimental values of $T_{N}$. and $J$ in Eqs. (5.11) and (5.13).

The fact that we observe no evidence for 3-D magnetic order in our samples above $T=2 \mathrm{~K}$ would be consistent with the value of $T_{N}=0.028 \mathrm{~K}$ from Eq. (5.11), calculated assuming only interchain dipolar magnetic coupling (Table 5.4). The much larger value of $T_{N}=5 \mathrm{~K}$ reported in Ref. [90] would thus imply that there is interchain coupling, in addition to that due to the magnetic dipole interaction, in $\mathrm{Sr}_{2} \mathrm{CuO}_{3}$ (see Table 5.3). Additional measurements, including magnetic susceptibility, specific heat and neutron diffraction, to temperatures lower than $2 \mathrm{~K}$ 
would be of great interest in the study of 3-D magnetic order in this system.

We note here that any long range three dimensional antiferromagentic structure adopted by $\mathrm{Sr}_{2} \mathrm{CuO}_{3}$ must be one in which the $\mathrm{Cu}^{+2}$ moments order antiferromagnetically within each chain; that is, a ferromagnetic intrachain interaction with an antiferromagnetic interchain interaction would not be consistent with our magnetic susceptibility data. Our low temperature structural data provide conclusive evidence that the magnetic properties of this material are not influenced by complications such as structural phase transformation or stablization of a spin-Peierls state, as occurs in $\mathrm{CuGeO}_{3}$ [113]. A number of other spin $1 / 2$ Heisenberg chains based upon $\mathrm{Cu}^{+2}$ have also been reported [98],[99],[106], but all of these compounds are not oxides and have much smaller values of $J$. The value measure here, $|J| / \mathrm{k}_{B} \approx 1300 \mathrm{~K}$, is quite large and comparable to that measured in the 2-D cuprate systems. It should be pointed out here that in the layered cuprate literature the prefactor of the sum over nearest neighbor pairs in the Hamiltonian of Eq. (5.1) has the factor of two missing. Thus one should compare $2 J(2600 \mathrm{~K})$ for $\mathrm{Sr}_{2} \mathrm{CuO}_{3}$ with $J(\sim 1500 \mathrm{~K})$ in the layered cuprate literature. It is an open question why the nearest neighbor $\mathrm{Cu}$-Cu superexchange interaction in $\mathrm{Sr}_{2} \mathrm{CuO}_{3}$ is significantly larger than in the antiferromagnetic layered cuprate systems.

Finally, the evolution of the magnetic and electronic dimensionalities in $\mathrm{Sr}_{2} \mathrm{CuO}_{3+\delta}$ as a function of $\delta$ is a very important subject for future study. Although there are apparently several superconducting phases $[85],[114],[115]$ in this system, their crystal structures, particularly the precise oxygen ordering arrangements, have yet to be determined. Thus the intriguing question of how a 1-D antiferromagnet evolves into a high temperature superconductor in this system upon doping remains to be 
answered.

\section{Conclusion}

Magnetic susceptibility measurements for $\mathrm{Sr}_{2} \mathrm{CuO}_{3 \pm \delta}$ were made from $2 \mathrm{~K}$ to $800 \mathrm{~K}$, and a strong dependence upon oxygen content $(\delta)$ was observed. Samples synthesized under oxygen, followed by various nitrogen treatments, exhibited markedly different Curie-Weiss-type terms, and we discuss possible origins for this behavior. High temperature magnetic susceptibility measurements for the sample with the smallest Curie-Weiss-type term clearly show the increase with temperature expected from the Bonner-Fisher model for a spin-1/2 one dimensional Heisenberg antiferromagnet. This is the first direct experimental observation of 1-D magnetic behavior in this system. The in-chain superexchange coupling constant, as determined by a fit to the Bonner-Fisher model, is $|J| / \mathrm{k}_{\mathrm{B}} \approx 1300 \pm 200 \mathrm{~K}$, comparable to the values observed in the two dimensional layered cuprates. Estimates of the interchain magnetic interaction indicate that this material may be the best realization of a 1-D spin 1/2 Heisenberg antiferromagnet reported to date. Low temperature neutron and synchrotron $\mathrm{x}$-ray powder diffraction studies of $\mathrm{Sr}_{2} \mathrm{CuO}_{3}$ indicate that the low temperature structure of this system has Immm space group symmetry, the same structure reported at room temperature, indicating that this material, in contrast to $\mathrm{La}_{2} \mathrm{CuO}_{4}$, does not undergo any structural transformations upon cooling. The absence of crystallographic distortions precludes a magnetic anisotropy contribution from a Dzyaloshinsky-Moriya interaction, implying that $\mathrm{Sr}_{2} \mathrm{CuO}_{3}$ should be a nearly ideal spin $1 / 2$ antiferromagnetic Heisenberg chain compound, in agreement with the magnetic susceptibility results. A search for the presence of long range three 
dimensional antiferromagnetic order by magnetic neutron powder diffraction at temperatures as low as $1.5 \mathrm{~K}$ was not successful, although we estimate an upper limit for the size of the ordered moment which could have been detected to be $\sim 0.1 \mu_{B}$ per $\mathrm{Cu}^{+2}$ ion. 


\section{CHAPTER 6. MAGNETIC PROPERTIES OF $\mathrm{Sr}_{2} \mathrm{IrO}_{4}$}

Interest in spin- $-\frac{1}{2}$ two-dimensional Heisenberg antiferromagnets has greatly increased in recent years due to the discovery of high-temperature superconductivity in copper oxides which, in their insulating (undoped) phases, fall into this magnetic category. Although a number of oxides are known with the $\mathrm{La}_{2} \mathrm{CuO}_{4}\left(\mathrm{~K}_{2} \mathrm{NiF}_{4}\right)$ structure, no spin- $\frac{1}{2}$ two-dimensional antiferromagnetic oxides other than the cuprates have been thoroughly studied [101]. In order to deepen our understanding of the magnetic properties of the cuprates, and to expand the range of materials which might exhibit high-temperature superconductivity, we are investigating other metal oxides which would be expected to have one unpaired $d$ electron per metal ion. Here we describe our results for $\mathrm{Sr}_{2} \mathrm{IrO}_{4}$.

$\mathrm{Sr}_{2} \mathrm{IrO}_{4}$, which has the $\mathrm{K}_{2} \mathrm{NiF}_{4}$ structure, [116] has octahedrally coordinated $\mathrm{Ir}^{4+}$ ions. The electronic configuration of the $\operatorname{Ir}^{4+}$ ions is $[\mathrm{Xe}] 4 \mathrm{f}^{14} 5 \mathrm{~d}^{5}$. Due to the large crystal field and very large spin-orbit coupling, [117] the $\mathrm{Ir}^{4+}$ ion should be in the low-spin configuration $t_{2 g}^{5}$, with the ground state being ${ }^{2} T_{2}$. This state is split by spin-orbit coupling and the tetragonal crystal field (see below) into three Kramers doublets. Thus, the ground state for the $\mathrm{Ir}^{4+}$ ion in $\mathrm{Sr}_{2} \mathrm{IrO}_{4}$ is expected to be a spin- $\frac{1}{2}$ Kramers doublet, where the unpaired spin occupies a half-filled $d_{x y}$ band, similar to the situation found for $\mathrm{Cu}^{2+}$ in $\mathrm{La}_{2} \mathrm{CuO}_{4}$. [118] 
The sample was prepared by the Dupont group using standard solid-state synthetic techniques. [116] Magnetic susceptibility measurements were made with a Quantum Design SQUID magnetometer at Dupont and our Oxford Instruments Faraday balance. We note that preliminary electrical resistivity measurements indicate that $\mathrm{Sr}_{2} \mathrm{IrO}_{4}$ is an insulator, although further measurements on single crystals are highly desirable.

In Fig. 6.1(top) we show the temperature dependence of the magnetic susceptibility. There is a distinct magnetic transition at approximately $250 \mathrm{~K}$. In order to clarify the nature of this magnetic transition, in Fig. 6.1(bottom) we show magnetic hysteresis measurements for this sample at a temperature of $5 \mathrm{~K}$. There is a significant magnetic hysteresis, demonstrating that the magnetic structure has a ferromagnetic component. The magnitude of the ferromagnetic moment estimated from these data is far too small $\left(10^{-2}\right.$ Bohr magnetons per Ir ion) to attribute to full ferromagnetically aligned spin- $1 / 2 \mathrm{Ir}^{4+}$ ions, which should have a magnetic moment of 1.1 Bohr magnetons, perhaps renormalized downward by quantum fluctuations as in $\mathrm{La}_{2} \mathrm{CuO}_{4}$. Instead it seems more reasonable to attribute the ferromagnetic behavior to canted antiferromagnetism. The magnetic transition we observe is thus probably due to the appearance of long-range three-dimensional antiferromagnetic order of the $\mathrm{Ir}^{4+}$ moments. Furthermore, since $\operatorname{Ir}^{4+}$ should be a spin- $1 / 2$ ion, it has no first order single-ion magnetic anisotropy, and therefore the magnetic properties of $\mathrm{Sr}_{2} \mathrm{IrO}_{4}$ are expected to be Heisenberg like. If this is so, $\mathrm{Sr}_{2} \mathrm{IrO}_{4}$ would be, to our knowledge, the second spin-1/2 two-dimensional Heisenberg antiferromagnetic oxide reported (in addition to the layered copper oxides), although the layered vanadates (for example, $\mathrm{Sr}_{2} \mathrm{VO}_{4}$ ) may also belong to this category. Moun spin rotation measurements should 
be made to verify the magnetic ordering temperature.

Without single-ion anisotropy to cause moment canting, a different origin must be found for the appearance of weak ferromagnetism in this system. The most likely cause is a Dzyloshinsky-Moriya (DM) interaction which arises as a result of the structural distortion. The rotational distortion destroys the inversion center which exists midway between the $\mathrm{Ir}^{4+}$ ions along the (100) and (010) directions for I4/mmm symmetry and thereby permits an antisymmetric superexchange term to appear in the spin Hamiltonian due to the DM interaction. A DM interaction also generates canted $\mathrm{Cu}^{2+}$ moments as a direct result of the tilting distortion of the $\mathrm{CuO}_{6}$ octahedra in $\mathrm{La}_{2} \mathrm{CuO}_{4}$.

The strength of the Dzyaloshinsky-Moriya interaction is proportional to the amount of anisotropy in the Landé $\mathrm{g}$ factor, $|D| \sim(\delta g / g) J$, where $\delta g=g-2$. Since anisotropy of $g$ is a consequence of spin-orbit coupling, and the spin-orbit coupling in $\operatorname{Ir}^{4+}$ is large $\left(\sim 2000 \mathrm{~cm}^{-1}\right)$, it is expected that the Dzyaloshinsky-Moriya interaction in this $5 \mathrm{~d}$ system is comparable to or larger than that for $3 \mathrm{~d} \mathrm{Cu}^{2+}$ in $\mathrm{La}_{2} \mathrm{CuO}_{4}$ (spin-orbit coupling $\sim 800 \mathrm{~cm}^{-1}$ ). Furthermore, the relative magnitudes of the rotational distortions of the $\mathrm{IrO}_{6}$ octahedra in $\mathrm{Sr}_{2} \mathrm{IrO}_{4}\left(\sim 11^{\circ}\right)$ and the $\mathrm{CuO}_{6}$ octahedra in $\mathrm{La}_{2} \mathrm{CuO}_{4}\left(\sim 3^{\circ}\right)$ also lead one to expect a larger DM interaction in the former material. These facts are consistent with our observation of a canted moment in $\mathrm{Sr}_{2} \mathrm{IrO}_{4}$ one order of magnitude larger than that observed in $\mathrm{La}_{2} \mathrm{CuO}_{4}\left(\sim 10^{-3}\right.$ Bohr magnetons).

Fig. 6.1(top) also shows the high-temperature magnetic susceptibility of $\mathrm{Sr}_{2} \mathrm{IrO}_{4}$. The susceptibility decreases with temperature above the Néel temperature, but not in a fashion consistent with Curie-Weiss behavior. Similar data above the Néel tem- 

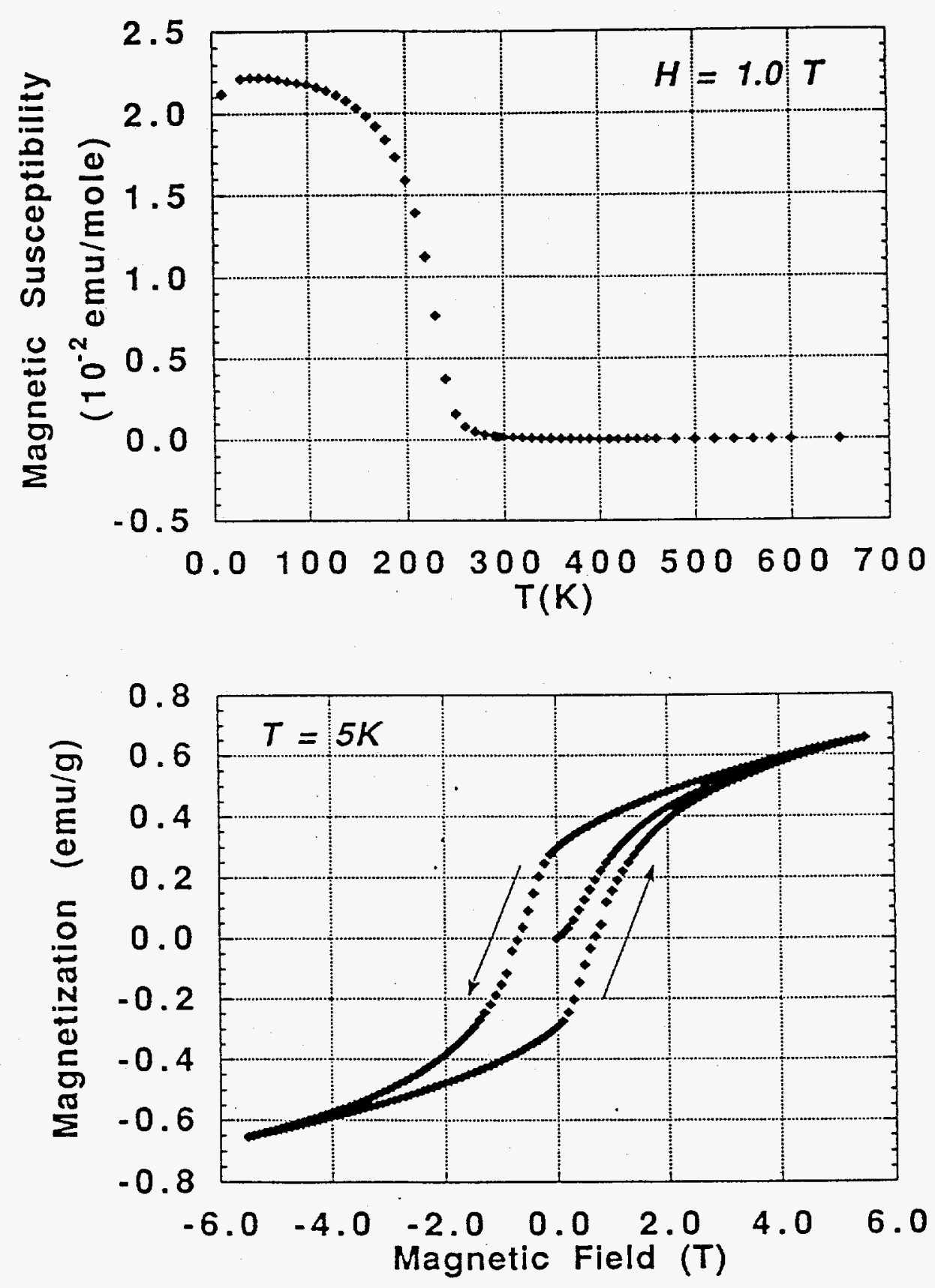

Figure 6.1: (Top) Magnetic susceptibility versus temperature $T$, data measured with a Faraday balance from 5 to $650 \mathrm{~K}$. (Bottom) Magnetic hysteresis curve measured with a SQUID magnetometer at $5 \mathrm{~K}$. The arrows indicate the direction in which the field is changing. 
perature in $\mathrm{La}_{2} \mathrm{CuO}_{4}$, which also do not follow a Curie-Weiss temperature dependence, were interpreted by a theory which clarified the role of the canted weak ferromagnetism in determining the magnetic properties of that material at temperatures above and below the Néel temperature. However, in contrast to the situation in $\mathrm{La}_{2} \mathrm{CuO}_{4}$, where the canted moments are antiferromagneticaly aligned in successive layers, our data [Fig. 6.1(top)] indicate that in $\mathrm{Sr}_{2} \mathrm{IrO}_{4}$ the canted moments in successive layers are ferromagnetically aligned. This is similar to the results reported for $L a_{2-x} \mathrm{Nd}_{x} \mathrm{CuO} \mathrm{O}_{4}$, where low-temperature structural phase transformations induce a magnetic transition from an antiferromagnetic to ferromagnetic interlayer arrangement of the $\mathrm{Cu}^{2+}$ spins.

It should be noted that a peak associated with two-dimensional short-range antiferromagnetic order does not appear in the susceptibility data above the Néel temperture in $\mathrm{Sr}_{2} \mathrm{IrO}_{4}$. Observation of such a peak would demonstrate the twodimensional nature of this magnetic system, which is expected on the basis of its layered structure and the in-plane superexchange interaction between $I r^{4+}$ ions. If a peak lies at a temperature above the maximum temperature measured, we can estimate a lower limit for the in-plane magnetic superchange of $J_{a b} \geq 650 \mathrm{~K}$, a value not quite as large as that of $\mathrm{La}_{2} \mathrm{CuO}_{4}\left(J_{a b} \sim 1500 \mathrm{~K}\right)$. Nevertheless, the magnetic properties of $\mathrm{Sr}_{2} \mathrm{IrO}_{4}$ have much in common with those of $\mathrm{La}_{2} \mathrm{CuO}_{4}$ and should offer fertile ground for further comparative study.

In conclusion, $\mathrm{Sr}_{2} \mathrm{IrO}_{4}$ is a two-dimensional spin-1/2 Heisenberg antiferromagnet. This material, which has a structural distortion which leads to weak ferromagnetism, has much in common with copper-oxide high temperature superconductors and should serve as an important example of this class of low-dimensional antiferro- 
magnetic oxides. In particular, study of $\mathrm{Sr}_{2} \mathrm{IrO}_{4}$ will further our understanding of the delicate connection between structural distortions and magnetism in such materials. 


\section{BIBLIOGRAPHY}

[1] J. G. Bednorz and K. A. Müller, Z. Phys. B 46, 189 (1986).

[2] M. K. Wu, J. R. Ashburn, C. J. Torng, P. H. Hor, R. L. Meng, L. Gao, Z. J. Huang, Y. Q. Wang, and C. W. Chu, Phys. Rev. Lett. 58, 908 (1987); R. J. Cava, B. Batlogg, R. B. van Dover, D. W. Murphy, S. Sunshine, T. Siegrist, J. P. Remeika, E. A. Rietman, S. Zahurak, and G. P. Espinosa, Phys. Rev. Lett. 58,1676 (1987).

[3] B. D. Josephson, Phys. Lett. 1, 251 (1962).

[4] L. D. Landau and E. M. Lifshitz, Statistical Physics (Pergamon Press, London, 1958).

[5] V. L. Ginzburg and L. D. Landau, Zh. Eksp. Teor. Fiz. 20, 1064 (1950).

[6] R. Rajaraman, An Introduction to Solitons and Instantons in Quantum Field Theory (North-Holland, New York, 1982).

[7] H. Zijlstra, Experimental Methods in Magnetism 2, ed. E. P. Wohlfarth (John Wiley \& Sons, New York, 1967), p. 94.

[8] L. Petersson and A. Ehrenberg, Rev. Sci. Instr. 56, 575 (1985), and references cited.

[9] L. Forró, J. R. Cooper, B. Rothaemel, J. S. Schilling, M. Weger, and K. Bechgaard, Solid State Commun. 60, 11 (1986).

[10] D. Wohlleben and M. B. Maple, Rev. Sci. Instr. 42, 1573 (1971), and references cited.

[11] For a review, see D. C. Johnston, J. Magn. Magn. Mater. 100, 218 (1991). 
[12] L. N. Mulay and M. Klemkowsky, High Temperature Superconductivity. The First Two Years, ed. R. M. Metzger (Gordon and Breach, New York, 1988), p. 165.

[13] S. A. Shaheen, R. Tucker, and X. Xu, J. Appl. Phys. 73, 5638 (1993).

[14] W. Mexner and K. Heinemann, Rev. Sci. Instr. 64, 3336 (1993).

[15] A. Wulfes, Ch. Böttger, J. Hesse, J. Sievert, and H. Ahlers, J. Magn. Magn. Mater. 104, 2069 (1991); J. Hesse, Ch. Böttger, J. Sievert, and H. Ahlers, Phys. Stat. Sol. (a) 135, 343 (1993).

[16] R. Srinivasan and S. Usha, J. Phys. E: Sci. Instrum. 19, 930 (1986).

[17] H. Zhou, Ph. D. Thesis, Univ. of Calif. San Diego (1988).

[18] U. Köbler and F. Deloie, Design and Operation of a Novel Faraday-magnetometer Using Superconducting Coils, Oxford Instruments.

[19] A. Roth, Vacuum Technology (North-Holland, New York, 1976).

[20] M. Knudsen, Ann. Phys. 31, 205 (1910); M. Knudsen, Ann. Phys. 32, 890 (1910); M. Knudsen, Ann. Phys. 33, 1435 (1910).

[21] Cryomagnetic System General Operating Procedures, Oxford Instruments.

[22] L. F. Mattheiss, E. M. Gyorgy, and D. W. Johnson, Jr., Phys. Rev. B 37, 3745 (1988).

[23] D. G. Hinks, B. Dabrowski, J. D. Jorgensen, A. W. Mitchell, D. R. Richards, and Donglu Shi, Nature (London) 333, 30 (1988); R. J. Cava, B. Batlogg, J. J. Krajewski, R. C. Farrow, L. W. Rupp, Jr., A. E. White, K. T. Short, W. F. Peck, Jr., and T. Y. Kometani, Nature (London) 332, 814 (1988).

[24] D. G. Hinks, D. R. Richards, B. Dabrowski, A. W. Mitchell, J. D. Jorgensen and D. T. Marx, Physica C 156, 447 (1988).

[25] B. Batlogg, R. J. Cava, L. W. Rupp, Jr., A. M. Mujsce, J. J. Krajeski, J. P. Remeika, W. F. Peck, Jr., A. S. Cooper, and G. P. Espinosa, Phys. Rev. Lett. 14, 1670 (1988).

[26] J. F. Zasadzinski, N. Tralshawala, D. G. Hinks, B. Dabrowski, A. W. Mitchell, and D. R. Richards, Physica C 156, 519 (1989).

[27] L. Krusin-Elbaum, R. L. Greene, F. Holtzberg, A. P. Malozemoff, and Y. Yeshurun, Phys. Rev. Lett. 62, 217 (1989). 
[28] Sreeparna Mitra, J. H. Cho, W. C. Lee, D. C. Johnston, and V. G. Kogan, Phys. Rev. B 40, 2674 (1989).

[29] Y. J. Uemura, V. J. Emery, A. R. Moodenbaugh, M. Suenaga, D. C. Johnston, A. J. Jacobson, J. T. Lewandowski, J. H. Brewer, R. F. Kiefl, S. R. Kreitzman, G. M. Luke, T. Riseman, and C. E. Stronach, Phys. Rev. Lett. 62, 2317 (1989).

[30] G. Aeppli, R. J. Cava, E. J. Ansaldo, J. H. Brewer, S. R. Kreitzman, G. M. Luke, D. R. Noakes, and R. F. Kiefl, Phys. Rev. B 35, 7129 (1987).

[31] F. N. Gygax, B. Hitti, E. Lippelt, A. Schenck, D. Cattani, J. Cors, M. Decroux, $\emptyset$. Fischer, and S. Barth, Europhys. Lett. 4, 473 (1987).

[32] D. R. Harshman, G. Aeppli, E. J. Ansaldo, B. Batlogg, J. H. Brewer, J. F. Caeolan, R. J. Cava, and M. Celio, Phys. Rev. B 36, 2386 (1987).

[33] W. J. Kossler, J. R. Kempton, X. H. Yu, H. E. Schone, Y. J. Uemura, A. R. Moodenbaugh, M. Suenaga, and C. E. Stronach, Phys. Rev. B 35, 7133 (1987).

[34] R. F. Kiefl, T. M. Riseman, G. Aeppli, E. J. Ansado, J. F. Carolan, R. J. Cava, W. N. Hardy, D. R. Harshman, N. Kaplan, J. R. Kempton, S. R. Kreitzman, G. M. Luke, B. X. Yang, and D. L. Williams, Physica C 143, 757 (1988).

[35] P. Birrer, J. E. Schirber, B. Morosin, and D. S. Ginley, Physica C 158, 230 (1989).

[36] Z. D. Hao, J. Clem, M. W. McElfresh, L. Civale, A. P. Malozemoff, and F. Holtzberg, Phys. Rev. B 43, 2844 (1991).

[37] S. Pei, J. D. Jorgensen, B. Dabrowski, D. G. Hinks, D. G. Richards, A. W. Mitchell, J. M. Newsam, S. K. Sinha, D. Vaknin, and A. J. Jacobson, Phys. Rev. B 41, 4126 (1990).

[38] E. H. Brandt, Phys. Rev. B 37, 2349 (1988).

[39] D. R. Harshman, L. F. Schneemeyer, J. V. Waszczak, G. Aeppli, R. J. Cava, B. Batlogg, L. W. Rupp, E. J. Ansaldo, and D. L. Williams, Phys. Rev. B 39, 851 (1989).

[40] For a review, see Y. J. Uemura, J. Appl. Phys. 64, 6088 (1988).

[41] Y. J. Uemura, B. J. Sternlieb, D. E. Cox, J. H. Kempton, R. F. Kief, S. R. Kreitzman, G. M. Luke, P. Mulhern, T. Riseman, D. L. Williams, W. J. Kossler, X. H. Yu, C. E. Stronach, M. A. Subramanian, J. Gopalakrishnan, and A. W. Sleight, Nature 335, 151 (1988). 
[42] U. Welp, W. K. Kwok, G. W. Crabtree, H. Claus, K. G. Vandervoort, B. Dabrowski, A. W. Mitchell, D. R. Richards, D. T. Marx, and D. G. Hinks, Physica C 156, 27 (1988).

[43] Y. J. Uemura, L. P. Le, G. M. Luke, B. J. Sternlieb, W. D. Wu, J. H. Brewer, T. M. Riseman, C. L. Seaman, M. B. Maple, M. Ishikawa, D. G. Hinks, J. D. Jorgensen, G. Saito, and H. Yamauchi, Phys. Rev. Lett. 66, 2665 (1991).

[44] T. A. Vanderah, editor, Chemistry of Superconductor Materials (Noyes, Park Ridge, NJ, 1992).

[45] D. Vaknin, S. K. Sinha, D. E. Moncton, D. C. Johnston, J. M. Newsam, C. R. Safinya, and H. E. King, Jr., Phys. Rev. Lett. 58, 2802 (1987).

[46] G. Shirane, R. J. Birgeneau, Y. Endoh, P. Gehring, M. A. Kastner, K. Kitazawa, H. Kojima, I. Tanaka, T. R. Thurston, and K. Yamada, Phys. Rev. Lett. 63, 330 (1989).

[47] For a review, see R. J. Birgeneau and G. Shirane, in Physical Properties of High Temperature Superconductors I, ed. D. M. Ginsberg (World Scientific, Singapore, 1989), p. 151.

[48] G. Shirane, Y. Endoh, R. J. Birgeneau, M. A. Kastner, Y. Hidaka, M. Oda, M. Suzuki, and T. Murakami, Phys. Rev. Lett. 59, 1613 (1991).

[49] P. W. Anderson, Science 235, 1196 (1987); J. R. Schrieffer, X.-G. Wen, and S.-C. Zhang, Phys. Rev. Lett. 60, 944 (1988); D. J. Scalapino, J. Phys. Chem. Solids 54, 10 (1993); P. Monthoux and D. Pines, Phys. Rev. B 49, 4261 (1994).

[50] P. W. Anderson, Phys. Rev. 79, 350 (1950).

[51] J. B. Goodenough, Magnetism and the Chemical Bond (Interscience, New York, 1963).

[52] A. Aharony, R. J. Birgeneau, A. Coniglio, M. A. Kastner, and H. E. Stanley, Phys. Rev. Lett. 60, 1330 (1988).

[53] D. Klemm, M. Letz, E. Sigmund, and G. S. Zavt, Phys. Rev. B 50, 7046 (1994), and references cited.

[54] B. Büchner, M. Breuer, A. Freimuth, and A. P. Kampf, Phys. Rev. Lett. 73, 1841 (1994). 
[55] F. Mizuno, H. Masuda, I. Hirabayashi, S. Tanaka, M. Hasegawa, and U. Mizutani, Nature 345, 788 (1990); H. Masuda, F. Mizuno, I. Hirabayashi, and S. Tanaka, Phys. Rev. B 43, 7871 (1991).

[56] F. Sapiña, J. Rodriguez-Carvajal, M. J. Sanchis, R. Tbáñez, A. Beltrán, and D. Beltrán, Solid State Commun. 74, 779 (1990).

[57] K. Seedhar and P. Ganguly, Inorg. Chem. 27, 2261 (1988).

[58] K. Okuda, S. Noguchi, K. Konishi, H. Deguchi, and K. Takeda, J. Magn. Magn. Mater. 104-107, 817 (1992).

[59] R. Hoffman, R. Hoppe, and W. Schäfer, Z. Anorg. Allg. Chem. 578, 18 (1989).

[60] R. Kipka and Hk. Müller-Buschbaum, Z. Naturforsh. 32b, 121 (1977); for a review, see Hk. Müller-Buschbaum, Angew. Chem. Int. Ed. Engl. 16, 674 (1977).

[61] M. T. Weller and D. R. Lines, J. Solid State Chem. 82, 21 (1989).

[62] E. F. Paulus, G. Miehe, H. Fuess, I. Yehia, and U. Lochner, J. Solid State Chem. 90, 17 (1991).

[63] M. A. G. Aranda and J. P. Attfield, unpublished.

[64] S. Eriksson, L. Johansson, L. Borjesson, and M. Kakihana, Physica C 162-164, 59 (1989).

[65] For a review, see W. Wong-Ng and R. S. Roth, Physica C (to be published).

[66] X.-L. Wang, J. A. Fernandez-Baca, Z. R. Wang, D. Vaknin, and D. C. Johnston, unpublished.

[67] D. Eckert, A. Junod, T. Graf, and J. Muller, Physica C 153, 1038 (1988); for a review, see R. A. Fisher, J. E. Gordon, and N. E. Phillips, J. Supercond. 1, 231 (1988).

[68] H. N. Migeon, F. Jeannot, M. Zanne, and J. Aubry, Rev. Chim. Min. 13, 440 (1976); H. N. Migeon, M. Zanne, F. Jeannot, and C. Gleitzer, Rev. Chim. Min. 14, 498 (1977).

[69] M. Wu, Q. Su, G. Hu, Y. Ren, and H. Wang, J. Solid State Chem. 110, 389 (1994). 
[70] D. C. Vier, S. B. Oseroff, C. T. Salling, J. F. Smyth, S. Schultz, Y. Dalichouch, B. W. Lee, M. B. Maple, Z. Fisk, and J. D. Thompson, Phys. Rev. B 36, 8888 (1987).

[71] R. N. de Mesquita, J. H. Castilho, G. E. Barberis, C. Rettori, I. Torriani, M. C. Terrile, H. Basso, and O. R. Nascimento, Phys. Rev. B 39, 6694 (1989).

[72] S. Petricek, N. Bukovec, and P. Bukovec, J. Solid State Chem. 99, 58 (1992).

[73] D. Vaknin, unpublished results.

[74] Z.-R. Wang, X.-L. Wang, J. A. Fernandez-Baca, D. C. Johnston, and D. Vaknin, Science 264, 402 (1994).

[75] P. W. Selwood, Magnetochemistry, $2^{\text {nd }}$ edition (Interscience, New York, 1956), p. 78.

[76] D. C. Johnston, T. Matsumoto, Y. Yamaguchi, Y. Hidaka, and T. Morakami, in Electronic Properties and Mechanisms of High Temperature Superconductors, edited by T. Oguchi, K. Kadowaki and T. Sasaki (Elsevier, Amsterdam, 1992), p. 301 .

[77] A. Bino, D. C. Johnston, D. P. Goshorn, T. R. Halbert, and E. I. Stiefel, Science 241, 1479 (1988).

[78] C. Kittel, Introduction to Solid State Physics, 6th edition (Wiley, New York, 1986).

[79] J. C. Bonner and M. E. Fisher, Phys. Rev. 135, A640 (1964).

[80] J. H. Van Vleck, Theory of Electric and Magnetic Susceptibilities (Oxford University Press, London, 1965).

[81] D. Gotteschi, A. Caneschi, L. Pardi, and R. Sessoli, Science 265, 1054 (1994), and references cited.

[82] R. Gottschall and R. Schöllhorn, Solid State Ionics 59, 93 (1993).

[83] G. Shirane, Acta. Cryst. 12, 282 (1985).

[84] A. H. Morrish, The Physical Principles of Magnetism (John Willey \& Sons, New York, 1965).

[85] Z. Hiroi, M. Takano, M. Azuma, and Y. Takeda, Nature 364, 315 (1993). 
[86] Chr. L. Teske and Hk. Muller-Büschbaum, Z. Anorg. Allg. Chem. 371, 325 (1969).

[87] The diffractometer was operated in the high resolution mode described by D. E. Cox, B. H. Toby, and M. M. Eddy, J. Phys. 41, 117 (1988).

[88] Y. J. Shin, E. D. Manova, J. M. Dance, P. Dordor, J.-C. Grenier, E. Marquestaut, J. P. Doumerc, M. Pouchard, and P. Hagenmuller, Z. Anorg. Allg. Chem. 616, 201 (1992).

[89] S. Kondoh, K. Fukuda, and M. Sato, Solid State Commun. 65, 1163 (1988).

[90] A. Keren, L. P. Le, G. M. Luke, B. J. Sternlieb, W. D. Wu, Y. J. Uemura, S. Tajima, and S. Uchida, Phys. Rev. B 48, 12926 (1993).

[91] M. T. Weller and D. R. Lines, J. Solid State Chem. 82, 21 (1989).

[92] Ch. Kruger, W. Reichelt, A. Almes, U. Konig, H. Oppermann, and H. Scheler, J. Solid State Chem. 96, 67 (1992); D. R. Lines, M. T. Weller, D. B. Currie and D. M. Osborne, Mater. Res. Bull. 26, 323 (1991).

[93] I. Dzyaloshinsky, J. Phys. Chem. Solids 4, 241 (1958); T. Moriya, Phys. Rev. 120, 91 (1960); T. Moriya, in Magnetism, edited by G. T. Rado and H. Suhl (Academic, New York, 1963), Vol. I, Chap. 3.

[94] Structural refinements were made using the GSAS software package [A. C. Larsen and R. B. von Dreele, LANCE, Los Alamos National Laboratory (1990)].

[95] D. Vaknin, S. K. Sinha, D. E. Moncton, D. C. Johnston, J. M. Newsam, C. R. Safinya, and H. E. King, Jr., Phys. Rev. Lett. 58, 2802 (1987); B. X. Yang, S. Mitsuda, G. Shirane, Y. Yamaguchi, H. Yamauchi, and Y. Syono, J. Phys. Soc. Jpn. 56, 2283 (1987).

[96] G. Aeppli and D. J. Buttrey, Phys. Rev. Lett. 61, 203 (1988).

[97] D. E. Cox, G. Shirane, R. J. Birgeneau, and J. B. MacChesney, Phys. Rev. 188, 930 (1969).

[98] M. T. Hutchings, E. J. Samuelsen, G. Shirane, and K. Hirakawa, Phys. Rev. 188, 919 (1969).

[99] L. J. De Jongh and A. R. Miedema, Adv. Phys. 23, 1 (1974); M. Steiner, J. Villain, and C. G. Windsor, ibid. 25, 87 (1976). 
[100] T. Thio, T. R. Thurston, N. W. Preyer, P. J. Picone, M. A. Kastner, H. P. Jenssen, D. R. Gabbe, M. Sato, K. Fukuda, S. Shamoto, Y. Endoh, K. Yamada, and G. Shirane, Phys. Rev. B 38, 905 (1988).

[101] R. J. Birgeneau and G. Shirane, in Physical Properties of High Temperature Superconductors I, edited by D. M. Ginsberg (World Scientific, Singapore, 1989), Ch. 3.

[102] E. A. Boudreaux and L. N. Mulay, Theory and Applications of Molecular Paramagnetism (Wiley, New York, 1976), p. 494.

[103] R. L. Carlin, Magnetochemistry (Springer-Verlag, Berlin, 1986).

[104] W. E. Estes, D. P. Gavel, W. E. Hatfield, and D. J. Godjson, Inorg. Chem. 17, 1415 (1978).

[105] H. Ohta, N. Yamauchi, M. Motokawa, M. Azuma and M. Takano, J. Phys. Soc. Jpn. 61, 3370 (1992).

[106] C. Allgeier and J. S. Schilling, Phys. Rev. B 48, 9747 (1993).

[107] C. J. Glinka, V. J. Minkiewicz, D. E. Cox, and C. P. Khatak, AIP Conf. Proc. 10, 659 (1972); M. Eibschutz, R. C. Sterwood, F. S. L. Hsu, and D. E. Cox, AIP Conf. Proc. 10, 684 (1972).

[108] S. K. Satija, J. D. Axe, G. Shirane, H. Yoshizawa, and K. Hirakawa, Phys. Rev. B 21, 2001 (1980).

[109] D. Vaknin, S. K. Sinha, C. Stassis, L. L. Miller, and D. C. Johnston, Phys. Rev. B 41, 1926 (1990).

[110] T. Oguchi, Phys. Rev. 133, A1098 (1964).

[111] E. Montroll, Proc. Third Berkeley Symp. on Mathematical Statistics and Probability (University of California Press, Berkeley, CA, 1956), Vol. 3.

[112] M. J. Hennessy, C. D. McElwee, and P. M. Richards, Phys. Rev. B 7, 930 (1973).

[113] M. Hase, I. Terasaki, and K. Uchinokura, Phys. Rev. Lett. 70, 3651 (1993).

[114] P. Laffez, S. Adachi, H. Yamauchi, and N. Mori, in Proc. Int. Conf. "Superconducting Materials" (ICMAS-93), edited by J. Etourneau, J. B. Torrance, and H. Yamauchi (IITT International, Paris, 1993). 
[115] T. Ami and M. K. Crawford, unpublished data.

[116] J. J. Randall, L. Katz, and R. Ward, J. Am. Chem. Soc. 79, 266 (1957).

[117] K. W. H. Stevens, Proc. Royal Soc. London, Ser. A 219, 542 (1954).

[118] It should be noted that the unpaired spin for $\mathrm{Cu}^{2+}$ resides in an $e_{g} 3 d$ orbital $\left(d^{2}-y^{2}\right)$

[119] D. E. Cox, B. H. Toby, and M. M. Eddy, Aust. J. Phys. 41, 117 (1988). 